\title{
PROJECT HISTORY AND ANALYSIS REPORT
}

COOPERATING COUNTRY:

PROJECT TITLE:

PROJECT NUMBER:

STARTING DATE OF PROJECT:

COMPLETION DATE OF PROJECT:

PERIOD COVERED BY THIS REPORT:

\section{AFGHANISTAN}

HERAT-ISLAM QALA HIGHWAY

$306-12-310-084$

27 August 1966

25 October 1967

$1966 .,-1967-1968$

Prepared by:

U.S. ARMY ENGINEER DIVISION, MEDITERRANEAN

U:S. ARMY CORPS OF ENGINEERS

A.I.D.

Reference Center

Room 1656 NS 


\section{LIST OF APPENDICES}

I. Construction Progress Chart and General Data

II. Materials

III: Culvert Schedule, Plan of Drainage and Dips

IV. Total Quantities of Work

V. Project Agreement

VI. Participating Agency Service Agreements

VII. Preventive Maintenance and Improvement Program

VIII. Typical Road, Bridge and Dip Sections

IX. Selective Meteorological Data

X. List of Government-Furnished Equipment

XI. Corps of Engineers Staffing Table

XII. Construction Contract Modifications

XIII. Finding of Fact 
above disputed Kashmir, shaped like a hand with its fingers touching Sinkiang Province in China.

Although annual rainfall throughout the country averages only 15 inches, the runoff from melting mountain snow has resulted in devastating floods in certain localities and because of the precipitous slopes and meager vegetation in many areas, moderately severe local rainstorms create flash floods of great intensity but short duration.

Afghanistan is extremely mountainous. The major mountain range, the Hindu Kush, lies in the center of the country and causes the routes of communication to pass around it north and south. Herat lies at the junction of the northern and southern routes, just at the west end of the Hindu Kush mountains. The major highway system forms a loop around the country with spurs radiating out to connect it with neighboring countries. There are three connecting roads to the USSR, two to Pakistan and one to Iran. The previous U.S.-sponsored or assisted road building prograns improved the two major links with Pakistan and the portion of the loop in the southeast. The USSR has built the southwestern section of the loop and the three connecting links with Russia; it is currently working on the northeastern sector of the loop.

The Governments of the United States and Afghanistan reached a "General Agreement for Technical Cooperation" on 7 February 1951. This was followed by a "Technical Cooperation Program Agreement" of 30 June 1953 (amended 20 December 1960) and an "Economic Cooperation Agreement" of 23 June 1956. Pursuant to the purposes of the se agreements and as a part of its aid to Afghanistan, the United States agreed to provide assistance in the road construction program under the first Five-Year Plan. This assistance was to be grant-financed and in the form of commodities, equipment, and technical advice and assistance for construction of certain selected parts of the proposed highway network in the eastern part of the country. In furtherance of this program, the U.S. International Cooperative Administration (ICA), which later became the U.S. Agency for International Development (AID), entered into Project Agreements (PRO AGS) with the Royal Government of Afghanistan (RGA) for improvement and maintenance of national roads.

Under the first Five-Year Plan for the development of the economy of Afghanistan, prepared in 1957, it was anticipated that a countrywide modern highway network to provide connecting links with major cities and the borders of Pakistan and the Soviet Union would be constructed.

In May 1957, the ICA sponsored a study of the transportation system of Afghanistan. The earliest road construction achievement 
resulting from the Program Agreements was construction of a portion of the Kabul-Torkham Road which stretched from the capital. past Jalalabad to Torkham, which is near the Khyber Rass, A difficult section of this road from the Kabul end was successfully constructed under the direct superviston of the Afghanistan, Government. Formerly it took more than ten hours to go from Kabul to Jalalabad; it now takes less than three hours. A second undertaking was the construction of a modern highway from Kandahar to Spin Baldak, the other entry to the country from Pakistan. Another goal accomplished by these projects was the training of Afghanistan nationals for future road construction and maintenance.

In 1961 , construction was started on the road from Kabul to Kandahar and this 510-kilometer major link in the Afghan highway system was completed in 1966. This highway linked the two largest cities in Afghanistan as well as many smaller towns along the route. The travel time was cut from a grueling 22-hour drive in good weather to an easy six-hour drive. About 5,700 technicians and construction workers were trained and their skills made available to the national economy. The high quality of work and the character of the contractor's performance enhanced the image of the United States in Afghanistan.

Under a second Five-Year Plan, started in 1962, the Royal Government of Afghanistan proposed to construct a road from ierat to Is lam Qala on the border of Iran. This would put an important link in the overall highway system and give Afghanistan ready access to Iran. The desirability of a good road through Herat was clearly shown when the trade routes with Pakistan were closed in 1961. Unable to trade across the eastern border, Afghanistan did not have any economical route for trading across its western border. The main route to Iran from Herat to Meshed was a desert track unimproved and frequently cut by flash floods. The road was bumpy and long and could not sustain any large volume of traffic. A much better route was needed for Afghanistan to export her produce or attract international traffic.

Subsequent to the drafting of the second Five-Year Plan, a series of meetings were conducted between the Royal Government of Afghanistan officials and the Director of USAID/Afghanistan to discuss the Afghan proposals and consider those elements of the Plan which might qualify for U.S. assistance. The financial assistance of the U.S. Gorernment was offered in the form of a grant to cover the cost of the engineering work and a loan for the actual contract construction costs of the Herat-Is lam Qala Road.

Acting on the advice of USAID, the Royal Government of Afghanistan called on the U.S. Corps of Engineers to supervise the engineering, planning and construction of the proposed road. With wide experience in supervising all types of construction and an in-country force established and well versed in the local problems, the Corps of Engineers 
merchandise, and at a low cost.

2. Open new land for settlement to Increase area under cultivation and stimulate production. Encourage agricultural growth and manufacturing of goods.

3. Provide a continuous all-weather highway connecting major cities and trade centers of the country.

4. Provide an important link in trans-continental highway now under development in southern Asia.

5. Provide a good all-weather highway to Iran, to promote and stimulate trade and commerce with the free world.

6. Provide more frequent contact between government officials and the public which should promote social development and political cohesiveness.

7. Promote continuing friendship between the United States and Afghanistan.

\section{DESIGN OF HERAT-ISLAM QALA HIGHWAY}

\section{Background}

Under the second Five-Year Plan, started in 1962, the Royal Government of Afghanistan proposed to construct a road from Herat to Islam Qala on the border of Iran. This would put another link in the overall national highway network and give Afghanistan ready access to Iran.

At the suggestion of USAID/A, the RGA called on the Corps of Engineers to supervise the project from initial planning through final construction.

In 1963, the Corps of Engineers made an initial engineering reconnaissance which included a thorough study of the economy of the area and the advantages to be gained by different type of roads.

In the fall of 1964 , a contract was awarded to Louis Berger, Inc., to perform final alignment survey of the road, investigation of the foundation for bridge and drainage structures and to design the Hari River bridge.

By July 1956, the Corps of Engineers had completed the final design and specifications for the highway. 
Most of the permanent monuments established for construction guidance were missing or broken, necessitating the reestablishment of the planned centerline. Interestingly enough, these monuments were broken up by the nomads for the reinforcing steel that they contained. Design grades were modified to provide cover for relocated pipe culverts and swale at the low end of the dips.

The following design criteria were used:

Design Speed: $90 \mathrm{Km} / \mathrm{hr}$. This was reduced to as low as $40 \mathrm{Km} / \mathrm{hr}$ in several critical locations where excessive construction cost would have been incurred in adhering to the design speed.

Horizontal Curves: 5 degrees or 350 meters minimum radius as required for design speed of $90 \mathrm{~km} / \mathrm{hr}$ in snor and ice conditions. However, in several locations the radius is reduced to as low as 150 meters where excessive construction cost would have resulted by adhering to the design speed of $90 \mathrm{Km} / \mathrm{hr}$. The maximum curve is 8,000 meters radius over very short lengths.

Longitudinal Grade: 3.5 percent maximum in flat terrain and 4.5 percent in rolling topography. However, the grade had to be increased in several locations to as much as 7.6 percent where excessive construction cost would have been incurred by adhering to these criteria.

Minimum Passing Sight Distance: 564 meters

Minimum Stopping Sight Distance: 126 meters

Width of Roadbed:

7.9 meters

Width of Pavement:

6.2 meters

Width of Shoulders:

.85 meters

Width of Bridge:

8.0 meters

Width of Right-of-Way: $\quad 50.0$ meters

(Typical roadway section is shown in Appendix VIII of this report.)

Three types of drainage structures, in addition to the Hari River bridge, were utilized to allow passage of water from the mountains into the river without damaging the road: (1) concrete dip sections provided at large, but shallow, water courses where 
Engineer, Louis Berger, Inc, boring 38 holes of which 27 were sunk to a depth of 50 feet at the location of the proposed structures. Samples were taken and sent to the laboratory for tests. Classification tests, including Atterberg limits, gradation, hydrometer, moisture density, soaked CBR, water content, soundness, LA abrasion, specific gravity, absorption, stripping and petrographic analysis tests were performed in the Division Materials Laboratory on the samples submitted.

The climate in the area in which the road is located is characterized by moderately cold, wet winters and hot, dry summers. The weather data recorded by the Afghanistan Weather Bureau, from 1958 to 1961 , indicates a freezing index ranging from 11 to 38 degrees Fahrenheit.

\section{CONSTRUCTION OF THE HERAT-ISLAM QALA HIGHWAY}

\section{Background}

The second Five-Year Plan for the economic development of the economy of Afghanistan, started in 1962, included the construction of an all-weather road from Herat to the Iranian border at Islam Qala. On 9 March 1963, the USAID Project Implementation Order/ Technical for the feasibility study and design of a road was issued to the U.S. Army Corps of Engineers, Mediterranean Division. The initial reconnaissance and feasibility study were performed in 1963 by personnel of the Afghanistan Area, Gulf District and Mediterranean Division. The "Feasibility Study, Herat-Islam Qala Highway, Afghanistan" was issued by the Division on 8 October 1964.

A final survey to include route selection and detailed soils tests of the route was made between November 1964 and January 1965 by the firm of Louis Berger, Inc., under an architect-engineer contract with the Corps of Engineers.

Following completion of the basic design, the final "Design Analysis, Herat-Is lam Qala Highway, Afghanistan" was completed in January 1966. Specifications were prepared by March 1966, and amended three times in May, prior to the opening of bids by prospective contractors.

\section{Results of Proposals and Award of Contract}

Proposals were solicited on 22 April 1966 to be received for opening on 20 June 1966. Only one proposal was received from a Joint Venture consisting of the following companies: 
Oman Construction Company, Inc.

R. P. Farnsworth \& Company, Inc.

Wright Contracting Company

Morrison-Knudsen International Co., Inc.

J.A. Jones Construction Company

Peter Klewit Sons' Company

This Joint Venture, under the name of the Afghanistan Highway Constructors (abbreviated AHC), was at that time completing work on the Kabul-Kandahar Highway. Consequently, they had the necessary in-country personnel and equipment avallable to construct the HeratIs lam Qala Highway.

The proposal, in the amount of $\$ 12,211,767$, was in excess of the funds avallable for the work. In June and August 1966, conferences were held in Livorno, Italy, during which negotiations were conducted to reduce the price of the work to be accomplished within the avallable funding. During the course of the negotiations, the scope of work was changed to delete several items and to modify the design of others. Among items deleted were the requirement for a materials testing laboratory, reverse signal alarms on equipment and three bridges. Additional Government-furnished equipment and property were to be utilized, culverts changed from 1 meter reinforced concrete pipe (RCP) to field-determined size of corrugated metal pipe (CMP), CMP to be furnished by the Government from the excess remaining from the Kabul-Kandahar Road project, and design of a bridge that would utilize excess materials from the Kabul-Kandahar Road. This resulted in the issuance of Addendum No. 1 to the contract prior to its final approval and signature. The final contract price of $\$ 8,361,322$ was agreed to on 4 August 1966 and the contract signed on that date. This price included an agreement by the contractor to perform any additional required work up to $\$ 34,288$ if subsequent changes required were in excess of the $\$ 215,712$ contingency fund available.

\section{Project Construction}

The contract stated that work was to commence within 30 calendar days after receipt by the contractor of the Notice to Proceed and to be completed within 730 calendar days.

The Notice to Proceed was issued in Livorno on 4 August 1966 and the contractor commenced work on 27 August 1966. AHC, already active in Afghanistan and with sufficient equipment in country, was able to move to Herat and commence construction with a minimum of delay. Work was started from Station $0+000$, at the intersection with the existing Russian-built concrete road at the northern outskirts of Herat, and on the camp located 73 kilometers west of Herat along the road alignment. Until the camp was completed in November, 
the contractor leased a hotel in Herat. By the time that operations were curtailed due to inclement weather, the camp had been completed, footings poured for the bridge and box culverts, and earthwork emplaced for about $45 \mathrm{kilometers}$ of the road. At this time the project was 27 percent complete, as compared to a scheduled 17 percent.

From mid-December 1966 to March 1967, because of severe weather, operations were limited to the manufacture of crushed rock for base course, concrete aggregate and Double Bitumino!s Surface Treatinent (DBST). When the weather permitted, the contractor resumed construction.

As the contract had been changed to provide field-determined size CMP in place of the designed one-meter RCP for all culverts, the first portions of the road were provided with CMP of equivalent flow values as determined by area engineering personnel. In November 1966, a culvert schedule was compiled for the entire road which would utilize only the CMP available and provide at least the same flow values as the initial design. As then computed, these culverts vere to be paid for by linear meters of pipe installed, regardless of the diameter. Based on this assumption, many large diameter pipes vere to have been utilized and a large underrun was anticipated in this payment item. In March 1967, the contractor objected to this method of payment, contending that payment should be for equivalent fiow value as this was more directly related to the actual cost of constructing the headwalls and installing the pipe. With this rule applied to the culvert schedule, a considerable overrun would have developed, so another redesign of culverts was carried out. The resulting cost would then have been within a reasonable percentage of the original bid item cost.

As funds vere not available at the initiation of the contract for the pirchase of asphalt, POL and cement in Iran in a specias. Parter Letter of Credit, it was necessary for the contractor to estsblish his own funding arrangements in Iran for the purchase of these.itens. Only necessary cement and POL were to have been pirchased prio: to the establishment of the Letter of Credit. As no funds were arailable prior to 1 March 1967, no order for asphalt was placed until thea. This delayed the arrival on site until $11 \mathrm{July} 1957$. This initial order for asphalt was short by about 10 percent of the total required amount, and a reorder was initiated in July 1967. This shipment did not arrive in country until the road was almost complete; in order not to delay construction, 1600 barrels of $\mathrm{RC}-2$ and $\mathrm{MC}-1$ were borrowed from the Afghan Air Authority in September 1967, and the final i3 kilometers of the road surfaced with this.

By 15 April 1967, the contractor had proceeded as far as Kilometer 50 with all work but surfacing, and was working as far 
ahead as Station 72 . The work was proceeding rapidly and we 11 ahead of schedile. On 17 April 1967, the first of four flash floods occurred, stopping work and causing extensive damage to the completed road prism and drainage structures. A copy of the finding of fact made at the time by the Acting Area Engineer is attached as Appendix XIII. As a result of the damage incurred, it was deterinined that additional drainage capacity was required to handle the runoff experienced. A new design was prepared based on the experience of the flash floods and the very large volume of runoff encountered from them.

In May 1967, a conference was held in Livorno to negotiate the cost of the new scope of work and to determine a coirse of action. The initial tentative estimate of the additional cost of the anticipated redesign work was $\$ 3.3$ million. Subsequent negotiations reduced this price to $\$ 2,050,000$. This design would have provided reasonable assurance that similar extensive washouts would not occur on the completed road. In the reduced price, the contractor included several reduced unit prices and stipulated that these prices were only to be valid provided a notice to proceed was issued within 60 days. An additional purchase of $\$ 66,000$ in CMP was necessary to complete the work and this pirchase was authorized and expedited in order to keep culvert construction from greatly delaying road construction. The cost of repairs of damage alone, exclusive of the repairs to be done by the contractor at no cost to the Gcvernment, was about $\$ 153,000$.

Following discussions with personnel of AID/Washington, it was determined that only up to $\$ 1.25$ million could be nade available on a loan basis. Repair and redesign work was commenced from 3 taticn 73 backivards in early May 1967, and was completed throigh Station 25 when a Stop order was issued on all redesign work and the coatractor instructed to proceed only with new construction. In June, a further redesign negotiation conference was held during which the desigr of the entire remining work was reviewed and the additional scope of work altered to reduce the additional funds required to the available $\$ 1.25$ million. It was noted at this time that if these finds were not made available, the road would have to be terminated at the far side of the bridge, about Station 98, at which point tive available construction funds would be used up. The Royal Government of Aighanistan attempted to obtain a grant for the additional funds required since repair and redesign work had not been budgeted. However, the srant was not forthcoming.

As no funds were forthcoming by $24 \mathrm{July} 1957$, the date that the Government option for the quoted price of $\$ 1.25$ million terminated, a Stop order was issued for all work beyond Station 98 and the contractor concentrated on completing the work up to that point. 
Due to the general reduction in USAID funds, no additicnal loans or grants could be made to complete the redesign work. There was danger of this project becoming a "road to nowhere." Between $27 \mathrm{July}$ and 1 August 1967, a further redesign of the remaining portions of the road was done to provide a roud as cheaply as possible. The design criteria were reduced, drairage structures altered and changed wherever a savings co:lld be effected, the type of paving altered from a non-frost sisceptible one to a less expensive type and the grade lowered for several kilometers. The cost of the final redesign scope of work was within the available supplementary funds plus the small sum of the original contract cost not yet expended.

There was not sifficient CMP o: asphalt available for AHC to complete the additional work and the delay in delivery of these items would have precluded completion of the road in 1967 . is a considerable savings in overhead was included in the nezotiated price schedules in anticipation of completing work this year, it was imperative to find an interim source. The dfghan Air Authority agreed to loan the required asphalt from its stock anc to provide what 24-inch diameter CMP it had on hand. Some additional CMP owned by AHC was also provided. Two additional dip sections, with DSST surfaces, were substituted for still other culverts in the area fron Stations 8 to 11 . With these substitutions and the pife ioans, AHC was able to proceed with the remaining work undelayed by materia? shortages. The contractor went on an 11-hour day, $7-d$ yy par waek work schedule in order to accelerate progress.

On 14 September, the road was complete through Station o. 5 the near approach to the bridge. This section of the road wis inspected and accepted by Aldelmo Ruiz, Chief, Engineering Division, for USAID/Afghanistan, and Colonel Sayed Alem Shat, Projist Drector, Ministry of Public Works, Royal Government of Afghanistar (RG:), on 28 September 1967.

At 1750 hoirs on 9 october 1957, the final layer of cosect rock was placed to the Iranian border and by 12 octooer al? ikin: construction was complete. The final acceptance of the las sesinent. of the road by the above personnel was formalized on 25 octuber 1957 , ten months ahead of the originally scheduled completion diate

\section{Materials Testing}

In accordance with the negotiated change to the contract, the Corps of Engineers assumed the responsibility for testing materials and established a testing laboratory in Herat. Following Anerican Society of Testing Materials (ASTM) and American Society of state Highway officials (AASHO) standard procedures, gradation concrol tests were performed on proposed select borrow, subbase and basa materials, as well as concrete, fine aggregates and DBST materials. 
During the initial materials survey made by the contractor, samples were tested at the Ghazni laboratory and the Corps of Engineers performed duplicate tests for comparison. With these tests performed in Ghazni, approval of the sources of materials to start coustruction was not delayed.

The first concrete trial mixes were batched on 5 October 1966 as the contractor got ready to make his first concrete placement on Karese covers and bridge foundations. Regular tests were performed on the concrete aggregate prior to trial mixes and the contractor poured his first concrete without delay. A total of 41 trial mixes were made for structural and paving concrete. When the first concrete dip section was ready for placement, mix proportions had already been determined. A total of 155 sets of samples were made with a set ranging from 6 to 8 specimens. A grand total of 1116 samples were molded and tested.

AHC operated crushing and screening plants at Stations 3, 33, 76, 94 and 109 for the production of base rock, concrete coarse and fine aggregate, DBST coarse and cover aggregates, and graded filter materials. Most of the sand for this project was processed and washed at Station 33. A mobile laboratory was set up at every crusher site and screening plant site for gradation control and other simple tests; check samples were tested at the camp laboratory and, to guard against segregation in placement, in-place samples were taken.

The Double Bituminous Surface Treatment placement started on 15 July 1967. Laboratory technicians checked quantity control, while the supervisor handled quality control. A technician was also assigned to prime coat quality control. Samples of every bitumen used, $\mathrm{MC}-0, \mathrm{RC}-3, \mathrm{MC}-1$ and $\mathrm{RC}-2$ were sent to the Division Materials Laboratory for check tests. $M C-1$ and $\mathrm{XC}-2$ borrowed from the Afghan Air Authority were used in the last $13 \mathrm{kilometers}$ of the road.

The Materials Testing Laboratory operated with ten Afghan technicians and a Materials Engineer Supervisor. Three crews performed fleld density tests for subgrade, select borrow, subbase, culvert backfill, dikes and base course. A gradation technician was at the mobile laboratory and two technicians were at the main camp laboratory for maximum density-optimum noisture tests. The Afghan senfor laboratory technician was responsible to insure that concrete cylinders and beams were tested as scheduled. Abrasion tests and soundness tests on materials were also performed in the area labosatory.

TABULATION OF MATERIALS TEST FOR CONSTRUCTION CONTROL

Field Density Tests Performed: 
Subgrade

3530

Select Borrow

Culvert Backfill, Karese Cover Backfill, Dikes

Filter Course

Subbase Bottom Lift

Subbase Top Lift

Base Course

Gradation Tests in the Main Laboratory:

$\begin{array}{lr}\text { Side-Borrow in-place } & 230 \\ \text { Atterberg Limit Test } & 230 \\ \text { Maximum Density-Optimum Moisture } & 230 \\ & \\ \text { Select Borrow-Top Subgrade } & 89 \\ \text { Atterberg Limits } & 89 \\ \text { Maximum Density-Optimum Moisture } & 89 \\ \text { Hydrometer Analysis } & 89 \\ \text { Filter Course Gradations } & 350 \\ \text { Atterberg Limits } & 20 \\ \text { Hydrometer Analysis } & 350 \\ \text { Maximum Density-Optimum Moisture } & 20 \\ \text { Subbase, Bottom \& Top Lift Gradation } & 600 \\ \text { Atterberg Limits } & 100 \\ \text { Maximum Density-Optimum Moisture } & 100 \\ \text { Hydrometer Analysis } & 600\end{array}$

Gradation Tests Performed on Concrete Aggregates, DBST Aggregates and Base Course Material:

Concrete Aggregate-Coarse

Concrete Sand

DBST 3/4"

DBST Cover Aggregate

Base Course Material
438 ( $1 \frac{1}{2} "$ and $\left.3 / 4^{\prime \prime}\right)$

170

600

690

920

Other Tests:

Concrete Aggregate Specific \& Absorption 15

Concrete Fine Specific Gravity $\&$ Absorption

Abrasion Loss Tests

Unit Weight Determination PCC Aggt. 10

Unit Weight Determination DBST Aggt. 10

Stripping Test

Soundness Tests 


\section{Contractor Forces}

During the course of the contract, an average of sixteen Americans and twenty-three third-country nationals were employed by the contractor to supervise and augment an average local labor force of 931. During summer months labor forces averaged about 1400-1500. Many of the local laborers and virtually all supervisory and managerial personnel were experienced in this type of work, having just completed the Kabul-Kandahar Highway, and a maximum use was made of local laborers for foremen, skilled workers, and subprofessional positions. Only one piece of equipment, the final finish grader, was operated by a U.S. operator. A great deal of valuable experience in supervising construction operations was thus gained by Afghans, a technique that this country had few opportunities to develop before. These skilled workers and supervisors will remain an important resource to this country.

Throughout the life of this contract, AHC had excellent labor relations with the exception of a two-day walkout by some of their office engineers in March 1967. This dispute was over facilities furnished in the living quarters and was quickly resolved when the company agreed to provide electricity for lighting and outdoor washing facilities. This was the only organízed protest and may have been the first such move by construction workers in the country. Development of labor relations and understanding of labor organizing will be of value to Afghanistan.

\section{Supervision, Contract Administration and Organization}

The construction contract was supervised by the Afghanistan Area of the Corps of Engineers from Herat and the camp at Station 73 . The Afghanistan Area is a part of the U.S. Army Engineer Division, Mediterranean, with offices in Livorno, Italy. The Contracting officer was located in the Division office in Italy and functioned through the Afghanistan Area Engineer, his Contracting officer Representative (COR).

The Area Office employed a maximum of five Americans, six thirdcountry nationals and 24 Afghan employees, a total of 35 persorne 1. Maximum use was made of trained local personnel for those positions for which they were qualified, particularly in materials testing and office engineering. A staffing chart of the Area office is included as Appendix XI.

\section{Contract Modifications and $F$ ield Changes}

There were twelve numbered modifications to the basic contract, in addition to an initial amendment and three Memoranda for Record that altered the scope of work. These are summarized in Appendix XII. 
Two modifications resulted in reductions in the contract price; one was at no cost; the remaining nine were additions required by the redesign to improve drainage to meet on-site field conditions and to provide additional services or work not inftially anticipated, or better adaptation of the finished road to existing conditions. The amendment to the contract was issued prior to the Notice to Proceed and was a result of the negotiations conducted to reduce the design scope and overall contract price within the avallable funds. The first modification provided for both additions and deletions of contractor services and a more simplified design for culvert headwalls. However, the addition of contractor-furnished vehicles more than offset the deletions. The second modification was most practical and was at the request of RGA, who was concerned by the narrow paved portion of the road and desired as wide a paved section as could be obtained. The next two modifications we re required to correct shortages in personnel transportation and transportation of Government-furnished pipe that became apparent after work on the job commenced.

Modification No. 5 was the first of several changes which resulted in reducing the price of a drainage structure, initially a skew box culvert with large concrete headwalls, by a total of over $\$ 40,000$. This modification provided for CMP to replace the internal portion of the box structure. As a part of the redesign incorporated into Modification No. 6 and agreement reached in a Memorandum for Record (copy attached to Appendix XII), a further savings was effected by rechanneling the existing wash to reduce the skew from about $20^{\circ}$ to $70^{\circ}$ the reby eliminating the requirement for a large concrete headwal1. The initial structure would have cost $\$ 60,000$; the emplaced structure which provides equivalent protection, including culvert, headwalls and channel excavation, cost under $\$ 10,000$.

Numerous field changes were incorporated into the work to more closely fit the design to existing conditions and to utilize experience gained through working and residing in the area. The moxt extensive fleld change was made the subject of a Memorandum for Record (Appendix $X I I$ ) and involved several modifications to dip sections. A much more suitable finished product was obtained for no increase in cost. During the acceptance inspection of the first segment of the road, the representatives of the $R G A$ indicated several minor additions which they desired to the contract: additional road signs, kilometer posts and widening for toll stations. These were minor in nature and were incorporated as overruns to normal payment items. After their emplacement and before the transfer of the final increment of completed road, over 120 of the concrete guide posts were destroyed, mainly by local personnel hitting them with large rocks. These posts were all replaced by $A H C$ at no cost to the Government so that when the construction of the road was complete on 12 October 1967, all guide posts were 
standing. All replacement guide posts and the final few kilometers of posts were reinforced with reinforcing steel in the center hole and filled with Class $X$ concrete. This, too, was done at no cost to the Government, exclusive of the cost of the cement. These minor changes were the subject of another Memorandum for Record, Appendix XII.

The major modification, No. 6 , was the result of several redesigns of the drainage system as a result of the spring floods and road washouts. The primary consideration in the first two redesigns was to provide adequate drainage-carrying capacity for the damaged section of the road. The number of dips was increased from 10 to 32 , culvert capacity almost trebled, the interceptor ditches crosssectional area doubled in some locations, dikes were provided along interceptor ditch 15 for added protection against flooding, " $J$ " sections were redesigned to effect a much smoother merging flow of water and some degree of self cleaning, and stream channels were excavated and directed to drainage structures. Mounds were built on the downhill bank from the excavated earth to provide still further protection. The redesigned road, when completed, would suffer at most only minor damages in a series of flash floods such as occurred in April and May 1967. The major anticipated maintenance problem is silting up of the excavated stream' channels which would allow subsequent storms to seek their own channel. This, in turn, would allow the water to hit the road at some spot where no large drainage structure had been provided. The channels will require cleaning periodically.

In the negotiations which reduced the quoted price for redesign work from over $\$ 3.0$ million to a tentative $\$ 1.25$ million, the major modifications were the elimination of aprons on concrete dips, reduction in the amount of riprap and number of culvert pipes. Major price reductions were included and reduced overhead anticipated from a shorter working period. Much of this redesign was in road sections $\mathrm{Km} 0$ to 8,11 to 17 and 26 to 98 . The redesign work was generally complete in these areas prior to August. A major design feature employed on the inlet end of pipe culverts was to flare the edjes (providing a rounded entrance). This feature will markedly increase the carrying capacity of the culvert.

In late July, it was learned that the additional $\$ 1.25$ nillion would not be made available and the road would have to be completed with a minimum of additional funds. At this time the initial design and the redesign were both revised for the remaining portions and roughly $\$ 170,000$ worth of drainage features removed. This reduction could not possibly have been made without greatly increasing the probability of more severe damage to the roadway from flash floods, ponding and frost. All dip sections that had not yet been paved were reduced to a DBST surface with two cutoff walls and downstream 
dumped riprap protection. A dip section was replaced by a 144-inch diameter multi-plate pipe (MPP). (This was the one change that tended to improve the survivability of the road.) All remaining pavement was changed to Type $A$, the least expensive but more susceptible to damage from frost or ponded water. The grade was lowered for several kilometers, 114 to 123, where the land is quite flat. Although the road at the lower level will not be below the level of the expected flood, the road will be more vulnerable to, damage due to saturation of the embankment. The turn-around loop at the customs station was eliminated. Much handplaced riprap was deleted from the work. The completed road now has a greatly increased requirement for maintenance; it is likely that the DBST dip sections will require seasonal repairs.

\section{Maintenance Program}

The maintenance of this road has now been formally turned over to the RGA. In accordance with a verbal agreement between the Corps of Engineers and RGA Ministry of Public Works, a pamphlet was prepared by the Afghanistan Area Office setting out all the aspects of road maintenance necessary for the continued life of this road (Appendix VII). Particular emphasis was placed on the aspects of maintenance peculiar to this road, such as the importance-in keeping stream channels cleaned and the anticipated trouble in DBST dip sections. This, with other U.S. Army manuals on road maintenance, was provided to the local Director of Maintenance. Sufficient copies of these publications were provided for other personnel of RGA having responsibility for the maintenance program.

The road maintenance staff of the Royal Government of Afghanistan, Ministry of Public Works, has very little past experience. No maintenance other than sweeping of paved surfaces can be anticipated unless outside direction, equipment and supplies are furnished. Maintenance and repatr of the adjoining Russian-built road at Herat is directed and accomplished by Russian supervisors under contract. AID/Afghan has a maintenance program scheduled, but follow up on this project will be necessary.

A minimum amount of Government-furnished equipment from the road construction was retained at the site by the Public Works Department; this equipment is all ten to twenty years old and has been in continuous use. This is what is available to keep this road open - and this with no supply of spare parts and a budget that will not even purchase adequate diesel fuel. The original contract documents contained provisions for the U.S. contractor to purchase and turn over sufficient maintenance equipment for upkeep of the HIQ Highway. However, during negotiations to reduce the cost of the work, this provision was de le ted. 
If this road is to last, a maintenance program must be established, funded and manned soon.

\section{The Completed Highway}

The construction schedule for the contract established a completion date of August 1968. This date was set based on the assumption that this contractor might have to mobilize all his equipment and work force from the United States, establish his organization in Afghanistan and then commence work. It was also assumed that he would probably want to construct the road with the minimum amount of heavy equipment and a relatively small organization. The Afghanistan Highway Constructors with their organization in being and all of the equipment necessary for a much larger project already in the country, were able to begin the road construction almost immediately and remained well ahead of the established schedule. They were able to complete this task almost a year ahead of the scheduled date by utilizing their large spread of equipment throughout the construction season, by anticipating shortages and bottlenecks and by making timely provision for all necessary supplies and materials to be on site. When construction might have been delayed from a shortage of CMP or asphalt, temporary local resources were utilized to keep the job moving ahead. Initiative, resourcefulness, and good engineering management were needed to keep the large spread of equipment working productively. These were all provided by the capable supervisors of AHC. The tangible result of all this effort was that all major portions of the construction were completed by 15 October 1967, ten months ahead of schedule.

The Herat-Islam Qala Highway is a "nearly all weather" road which will be closed for a few hours following rainstorms while the water rushes down the wadis over the numerous dip sections. As the rains are very infrequent and many of the wadis quite wide, the 5.7 kilometers of dip sections represent a considerable savings over other possible types of drainage structures and will provide a reliable route for over 99 percent of the time.

The wearing surface is a Double Bituminous Surface Treatment 6.2 meters wide. Dip sections also are 6.2 meters in width. There is one bridge about 150 meters long which spans the Hari River 96.5 kilometers west of Herat.

The road includes 224 culvert structures, with a total equivalent pipe length of over 6,500 linear meters of one-meter diameter pipe. Construction included over 1.25 million cubic meters of earthwork, 17,000 square meters of grouted riprap and about 12,000 cubic meters of concrete. Typical specifications for material are given in Appendix II; the culvert schedule is given in Appendix III, and a detailed list of 
quantities of work accomplished and men and equipment used is given in Appendix IV.

The formal dedication ceremony was held on 14 March 1968 and was attended by:

\author{
Governor of Herat Province \\ Second Deputy Prime Minister \\ Public Works Minister Engineer \\ Interior Minister \\ Public Works Ministry Project \\ Director \\ Iranian Ambassador \\ UN Representative \\ U.S. Ambassador \\ Director USAID/A \\ Chief Engineer USAID/A \\ U.S. Embassy Defense Attache' \\ U.S. C.E. Contracting officer \\ U.S. C.E. Representative \\ Afghanistan Highway Constructors \\ Project Manager \\ Afghanistan Highway Constructors \\ Asst Project Manager
}

\author{
Mohamnad Seddiq \\ Abdullah Yaftali \\ Mohammad Hussein Masa \\ Mohammad Jmar Wardak \\ Sayyed Alam Shah \\ Mahmoud Feroughi \\ Arsene Shahbaz \\ Robert G. Neumann \\ Russe 11 S. McC lure \\ Aldelmo Ruiz \\ COL R. W. Hamelin \\ COL Denis $B$. Grace \\ Richard E. Phelps \\ J ames., Sumner \\ Joseph Kanady
}

Photos of the dedication ceremony are contained in the Pictorial Section.

\section{FUNDING AND COSTS OF THE PROJECT}

\section{Funding}

All engineering design work was paid for by U.S. grant-appropriated funds which were recommended by the President and approved by Congress. These funds were allotted to USAID who, in turn, issued a Project Implementation Order for Technical Services (PIO/T) to the Office of the Chief of Engineers (OCE) for prosecution of the work. In accordance with a directive of the Department of the Army, all work for USAID must be done on a reimbursed basis insofar as U.S. dollar funds are concerned. The $\mathrm{PIO} / \mathrm{T}$ is an authorization only and no funds are issued with it. Design and supervision were thus accomplished using MCA funds which were reimbursed. An exception to this method of funding was the amount of Afghanis required for the Area Office operations to pay the local payroll and for in-country costs. This money was made available directly from the AID/Afghanistan trust funds, and USAID provided the necessary finance administrative support. 
The construction of the road itself was paid for by a loan from the United States to Afghanistan. This was provided on a 40-year basis, with the first 20 years interest-free. Actual amounts of the loan for road construction are shown in the following letter-of-credit sums. In addition to this, the RGA provided the in-country Afghanis costs of 64,000,000 ( $\$ 876,712$ at the contract rate of 73 for one dollar) on a scheduled basis as agreed with the contractor.

A portion of the loan money was set aside to buy commodities in third countries, such as asphalt, POL and cement from Iran. This part of the loan was covered by a separate Barter Letter of Credit with the Irving Trust Company, New York. The major portion of the loan was made available by Letter of Credit with the First American National Bank of Nashville, Tennessee.

The separate barter letter was not set up until several months after the Notice to Proceed for the HIQ contract. As a result, AHC established its own Letter of Credit in the Bank Melli, Tehran, in order to make its Iranian purchases.

To provide for cost of the redesign work initiated as a result of the spring washout, an additional loan of $\$ 711,000$ which had initially been made to RGA for the purchase of fertilizer was transferred to the HIQ project account and is included in the following construction cost analysis.

ANALYSIS OF COSTS

Government \& Design Costs, Including S\&A:

$\begin{array}{ll}\text { FY }-65 & \text { Design Cost } \\ \text { FY }-65 & \text { Architect-Engineer Cost } \\ \text { FY }-66 & \text { Design Cost } \\ \text { FY }-67 & \text { Design Cost } \\ \text { FY }-67 & \text { S\&I Cost } \\ \text { FY }-68 & \text { Design Cost } \\ \text { FY }-68 & \text { S\&I Cost } \\ \text { FY }-69 & \text { S\&I Cost }\end{array}$

FINAL CONSTRUCTION CONTRACT COSTS:

Letter of Credit

Barter Letter of Credit

Afghanis $(64,000,000)$

$\begin{array}{lrr} & \$ & 68,832 \\ 155,668 \\ & 50,280 \\ & 15,826 \\ & 289,674 \\ & & 57 \\ & & 119,214 \\ & \\ & \\ \text { TOTAL } \$ \quad 701,412\end{array}$

TOTAL $\$ 701,412$

$\$ 7,668,801$

$\frac{616,053}{8,284,854}$

$\$ \frac{876,712}{9,161,566}$ 
A P P E N D I X I

CONSTRUCTION PROGRESS CHART

CONSTRUCTION PROGRESS REPORT

MONTHLY PERSONNEL COUNT

FINAL PAYMENT ESTIMATE, LINE ITEMS

FINAL PAYMENT ESTIMATE, BARTER ITEMS 


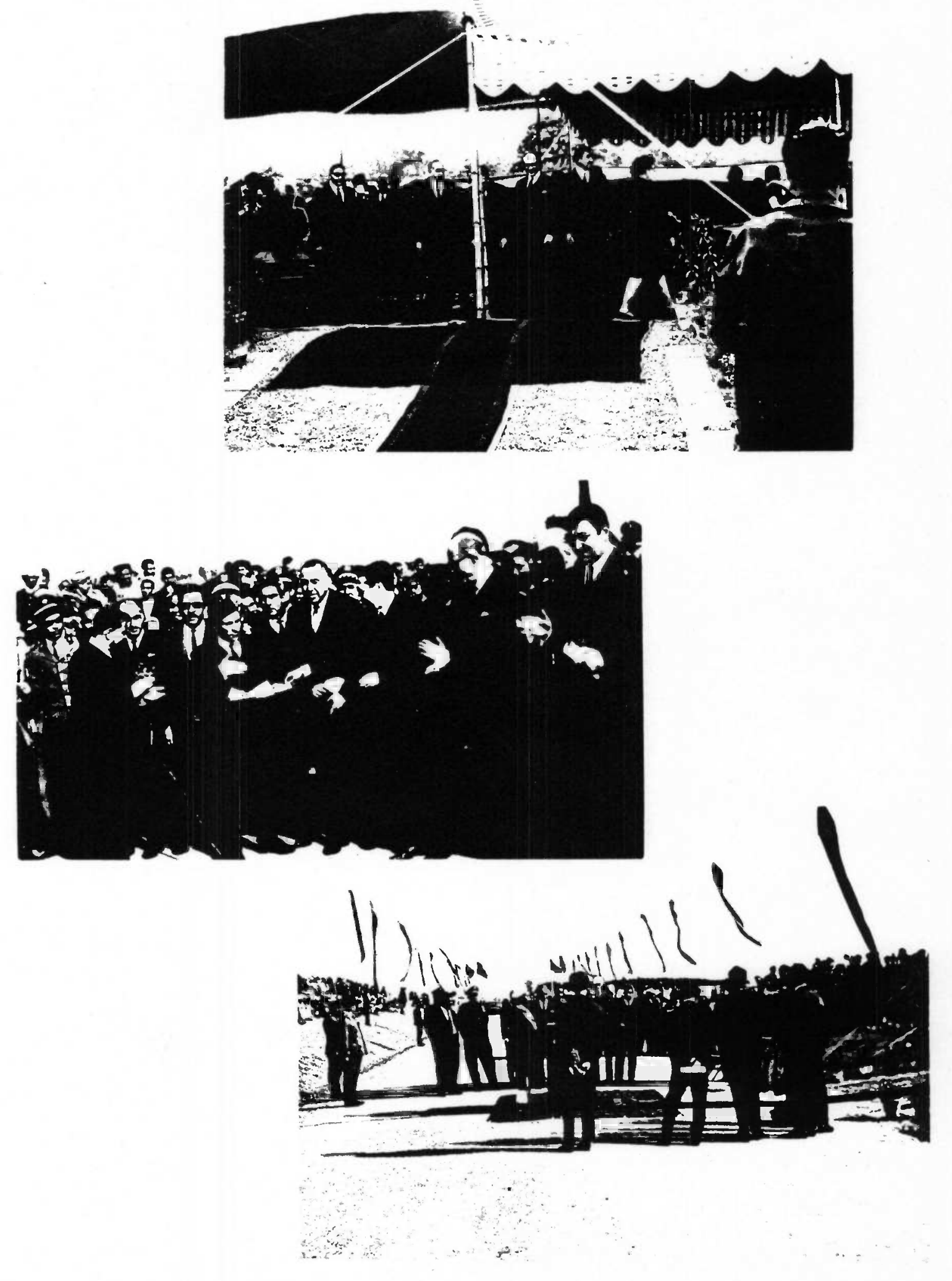

Views of HIQ Highway Dedication Ceremony 14 March 1968 


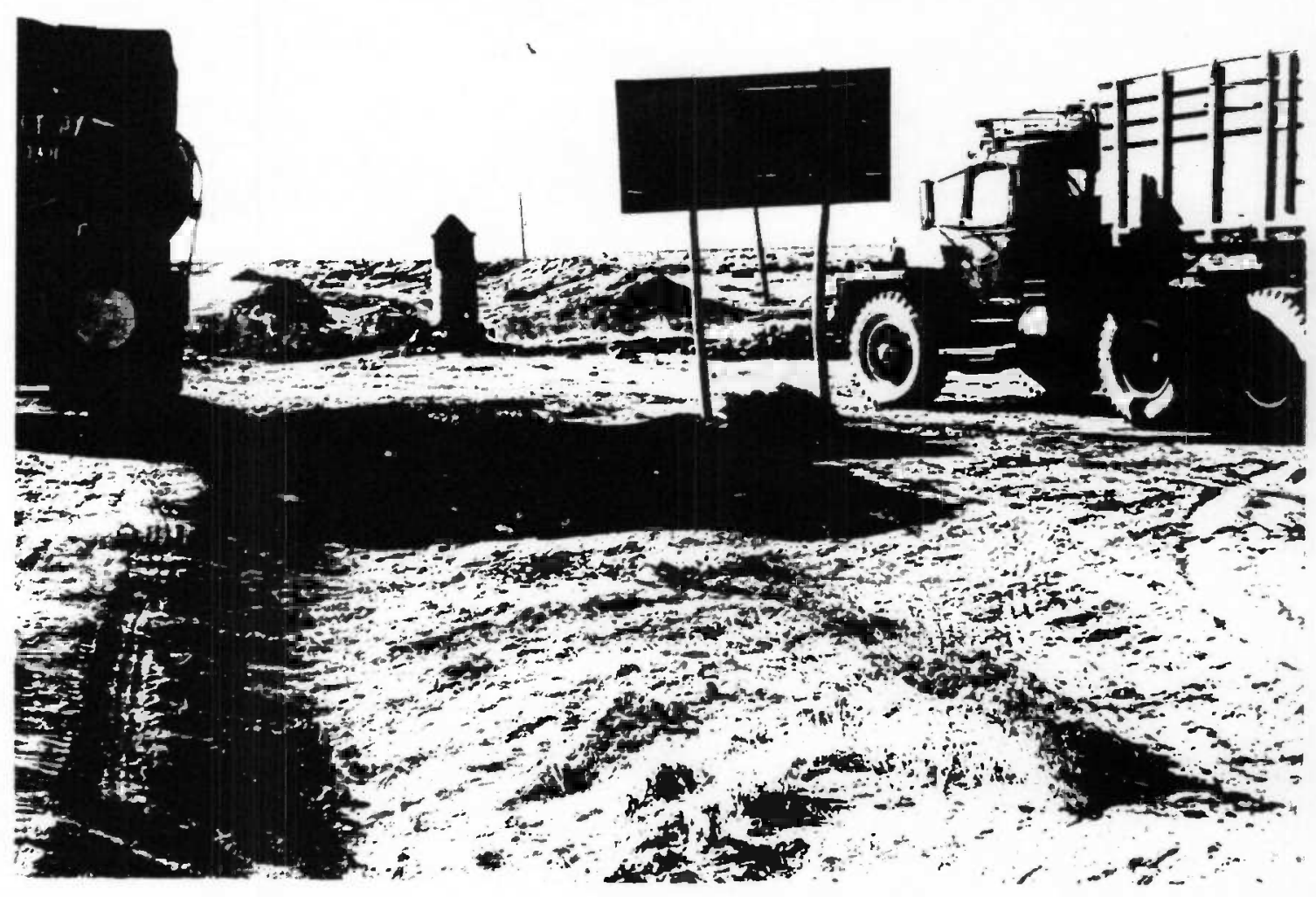

Ki lometer Station $123+00$

TOP - Before: old road junction at Afghanistan-Iranian border.

BOTTOM - After: Completed HIQ Highway at Afghanistan-Iranian border

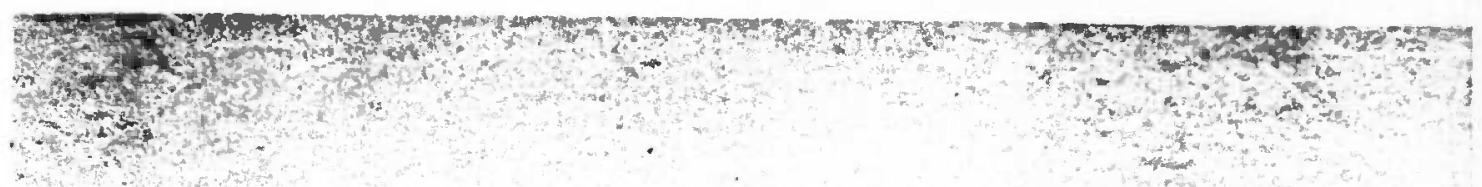

)

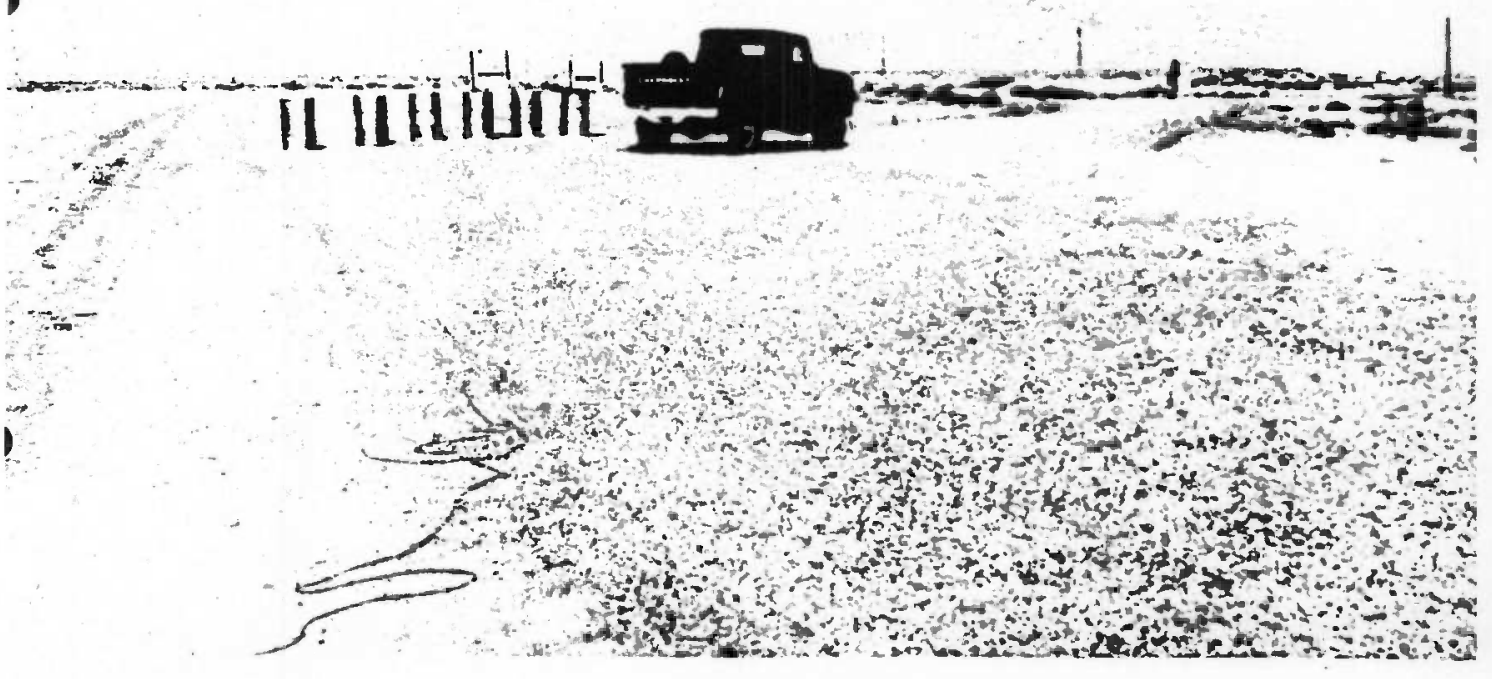




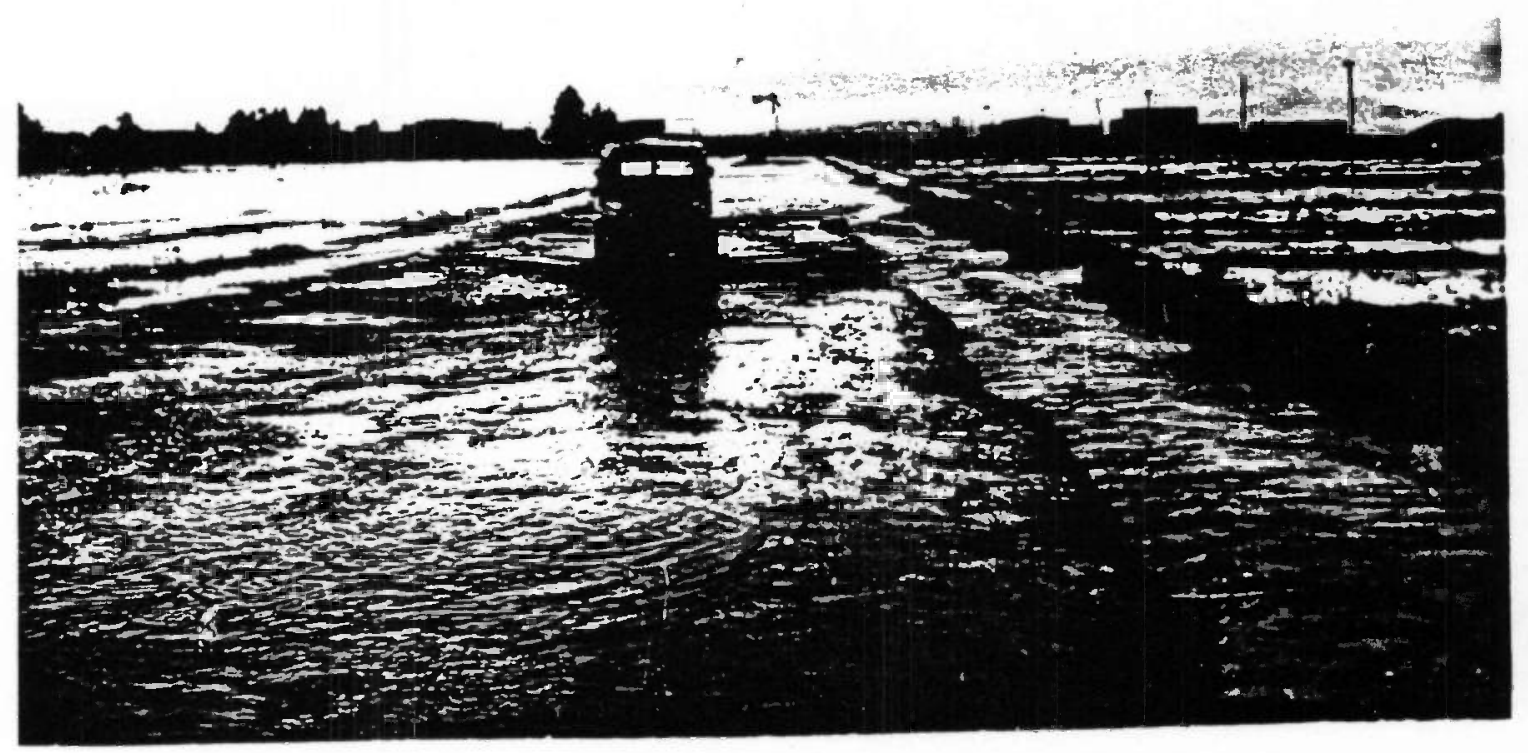

Kilometer Station $113+675$

TOP - Before: Flooded section of road at Is lam Qala during seasonal rains.

BоттоM - After: Ashpalt-paved dip section, 20 meters in length, to handle run-off during storms.

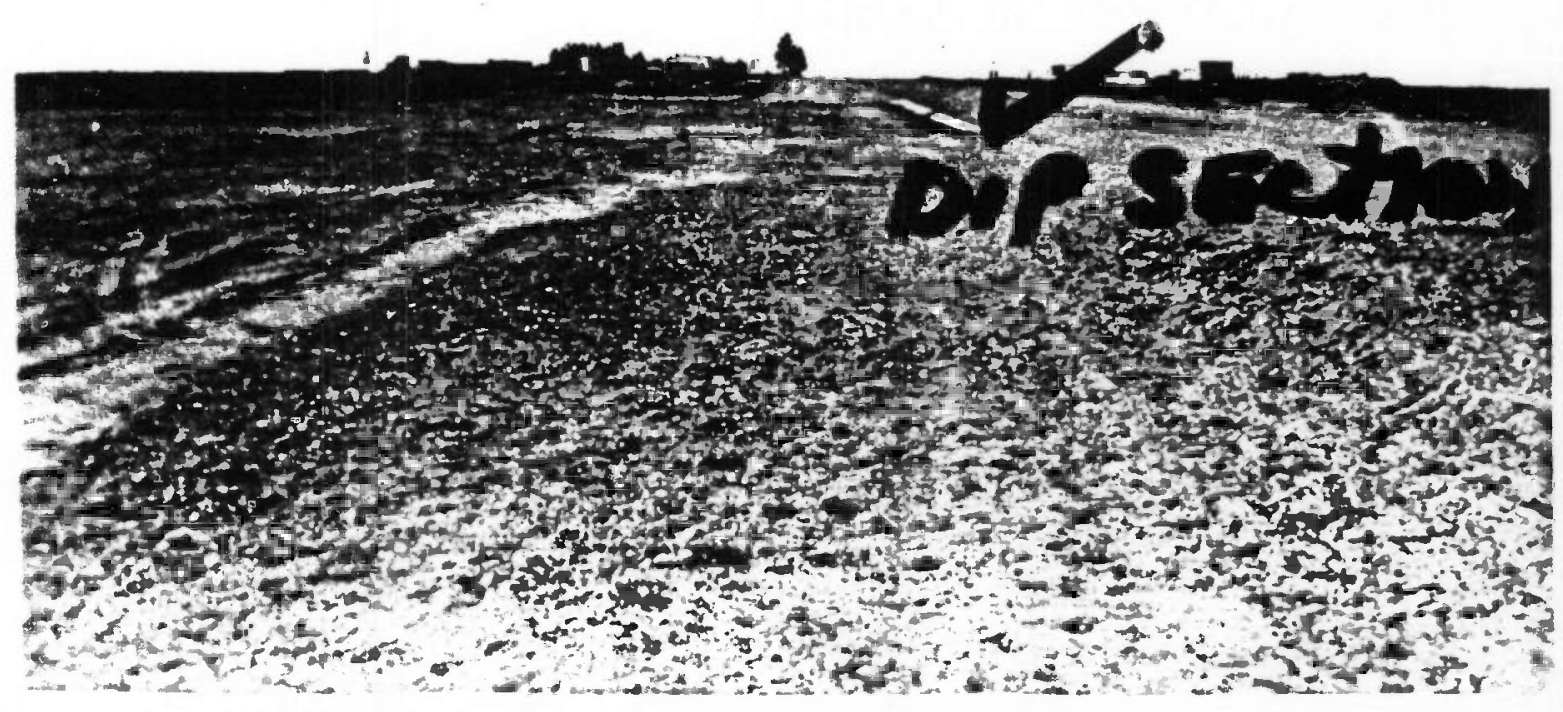




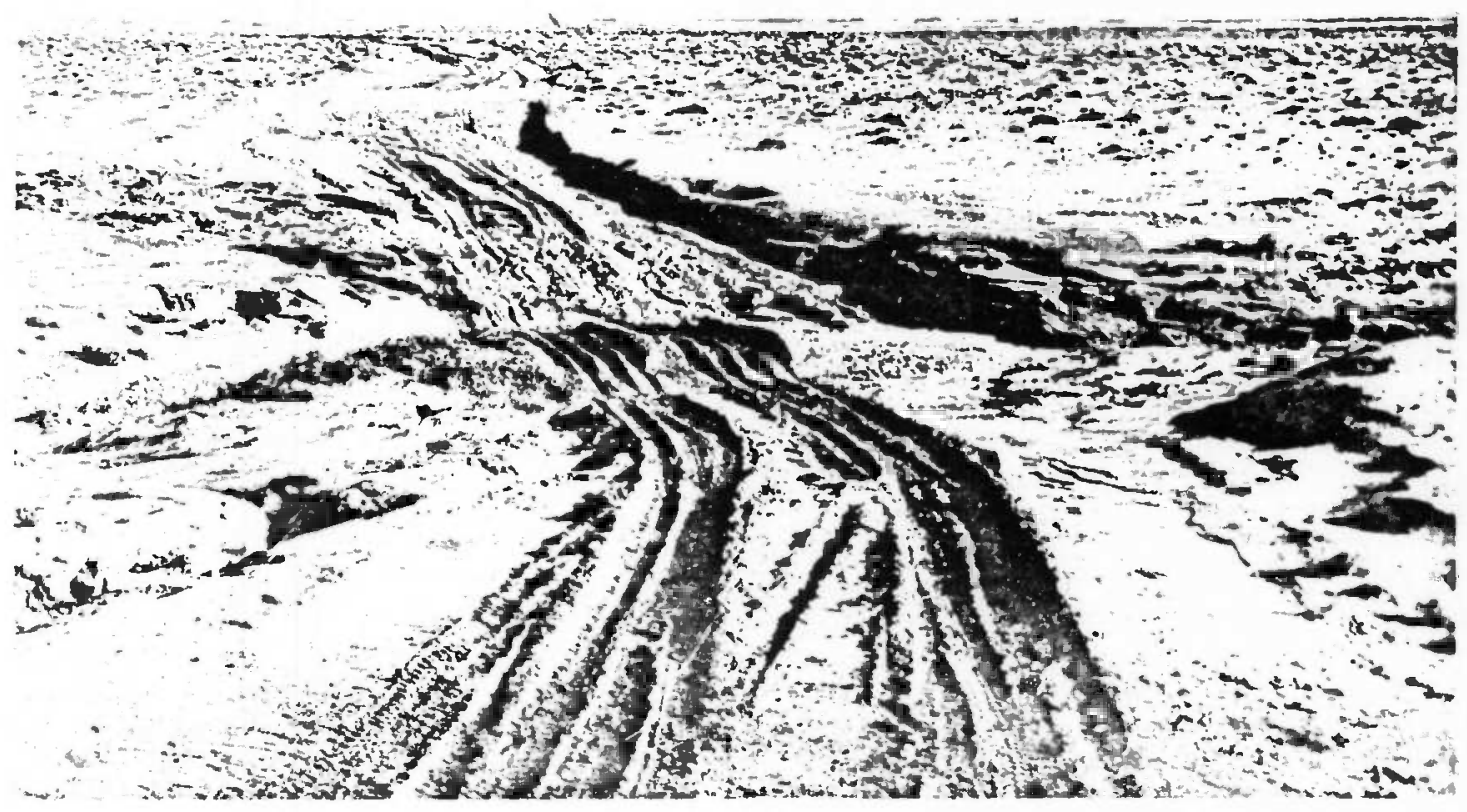

KiToneter Station $99+405$

Thp - Hione: Shows old road at wadi ater ratn. Looking north.

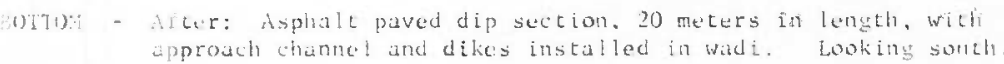

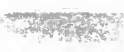

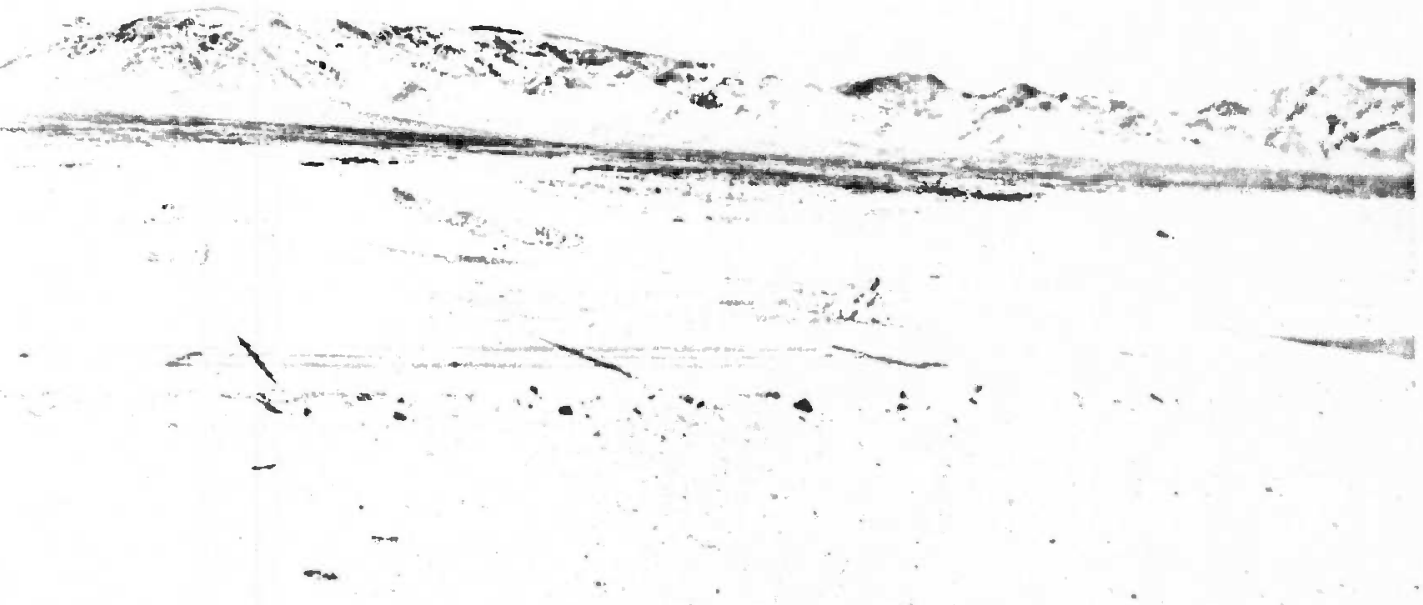




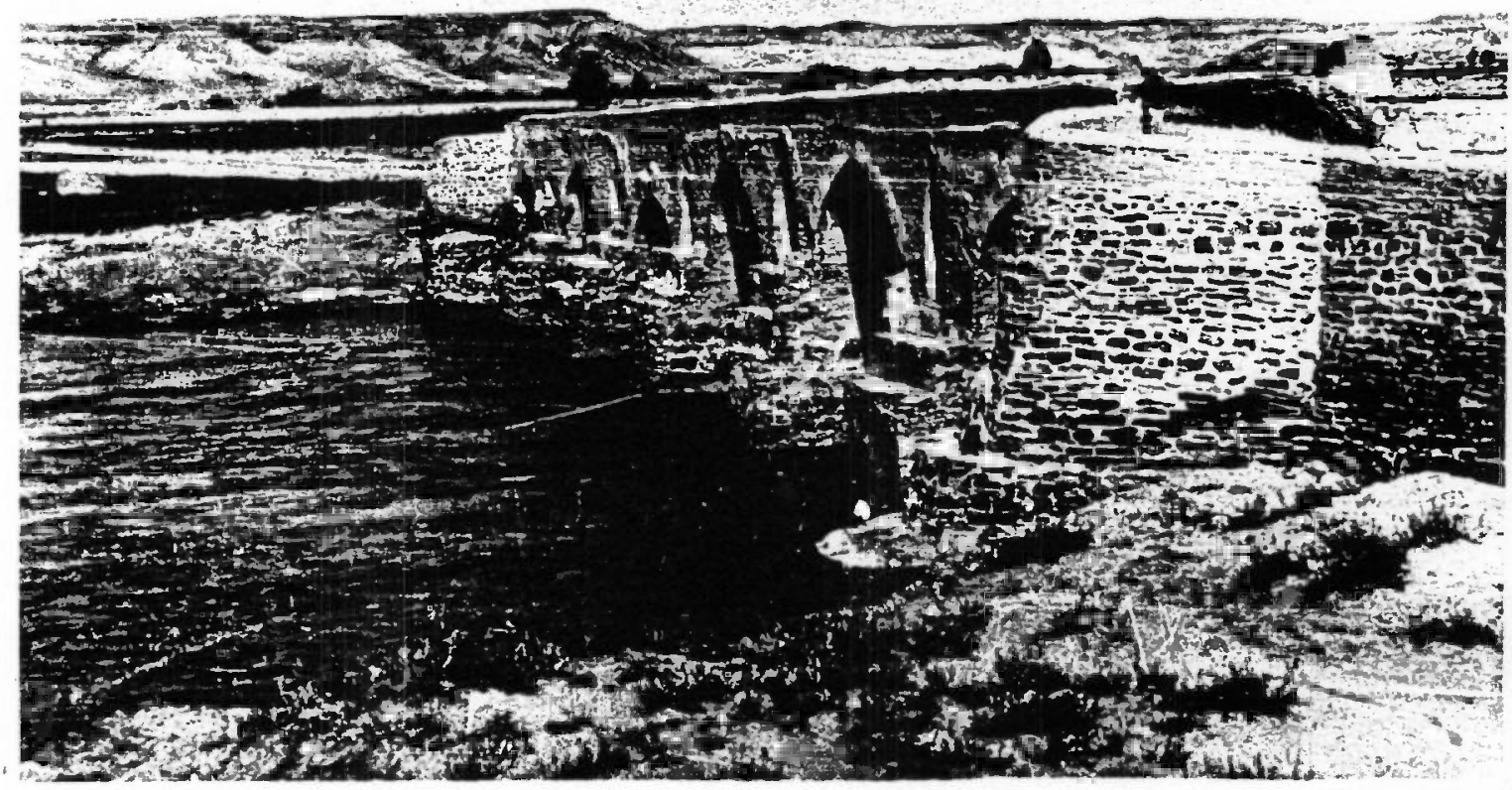

Kilometer Station $96+500$

TOP - Before: 0ld single-lane masonry bridge across Hari Rud (River)

BOTTOM - After: Two-lane, nine-span reinforced concrete bridge across Harj Rud (River).

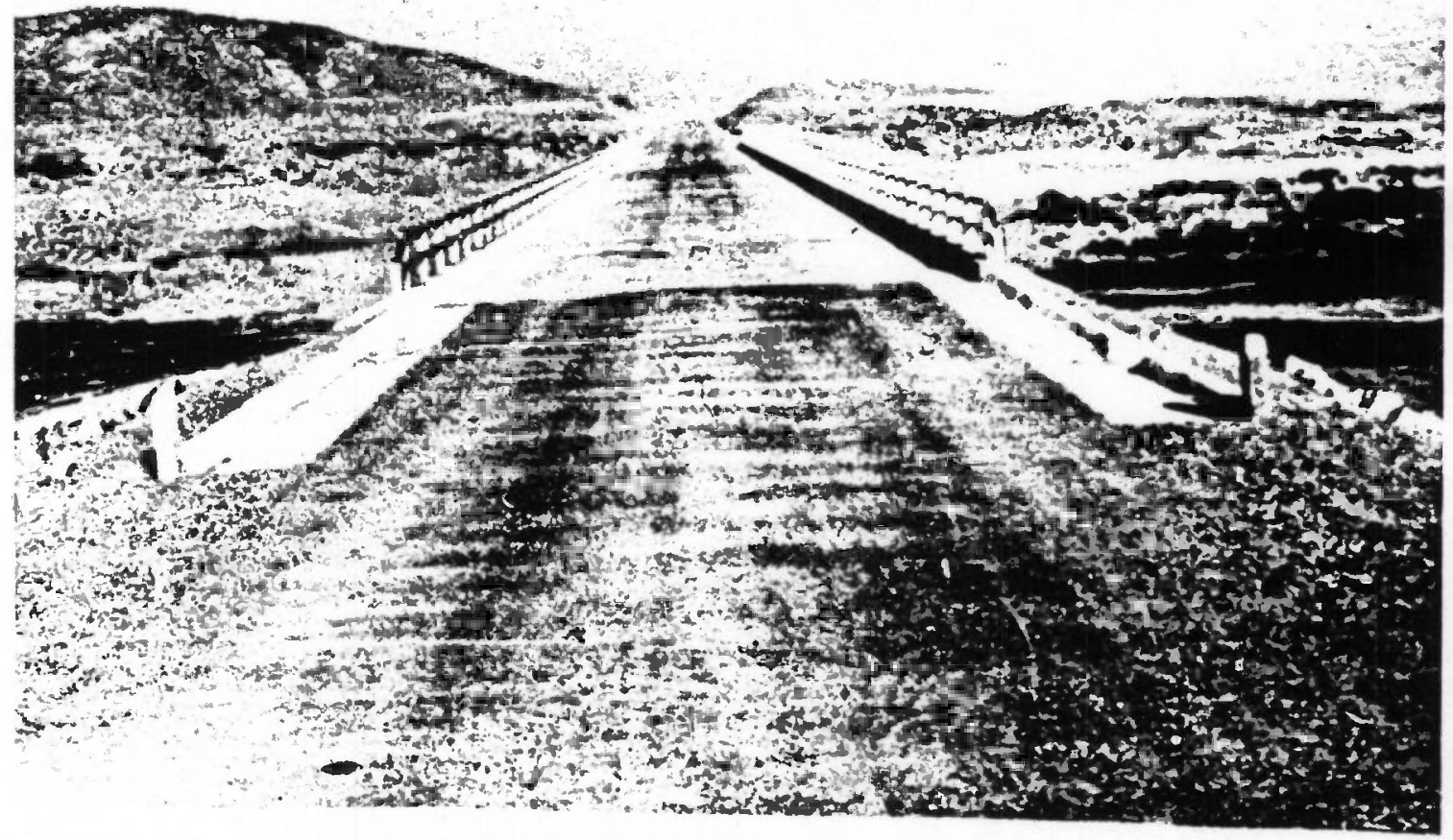




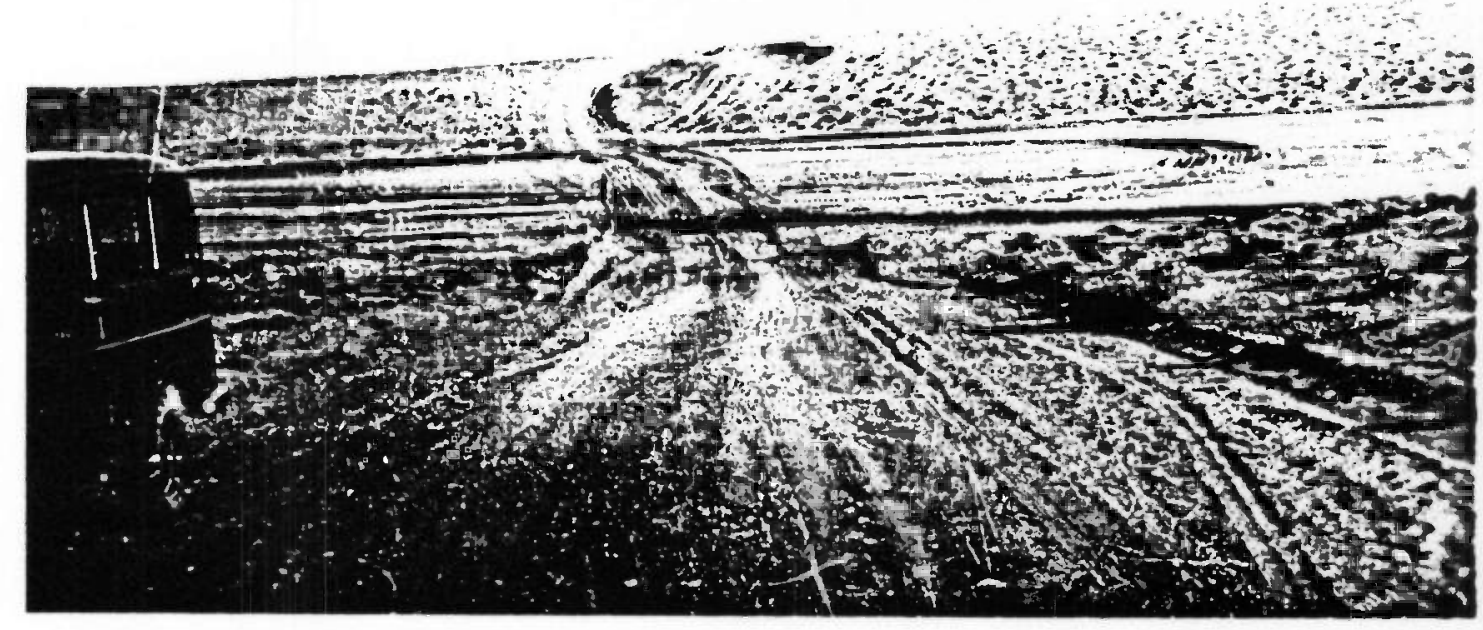

Kilometer Station $84+940$

Top - Fefort: Shows condition of old road through a wadi (drot sterm bed)

Borto - Arter: Concrete paved dip section in wad 140 meters in longti whith permits flash run-oín oi storm water.

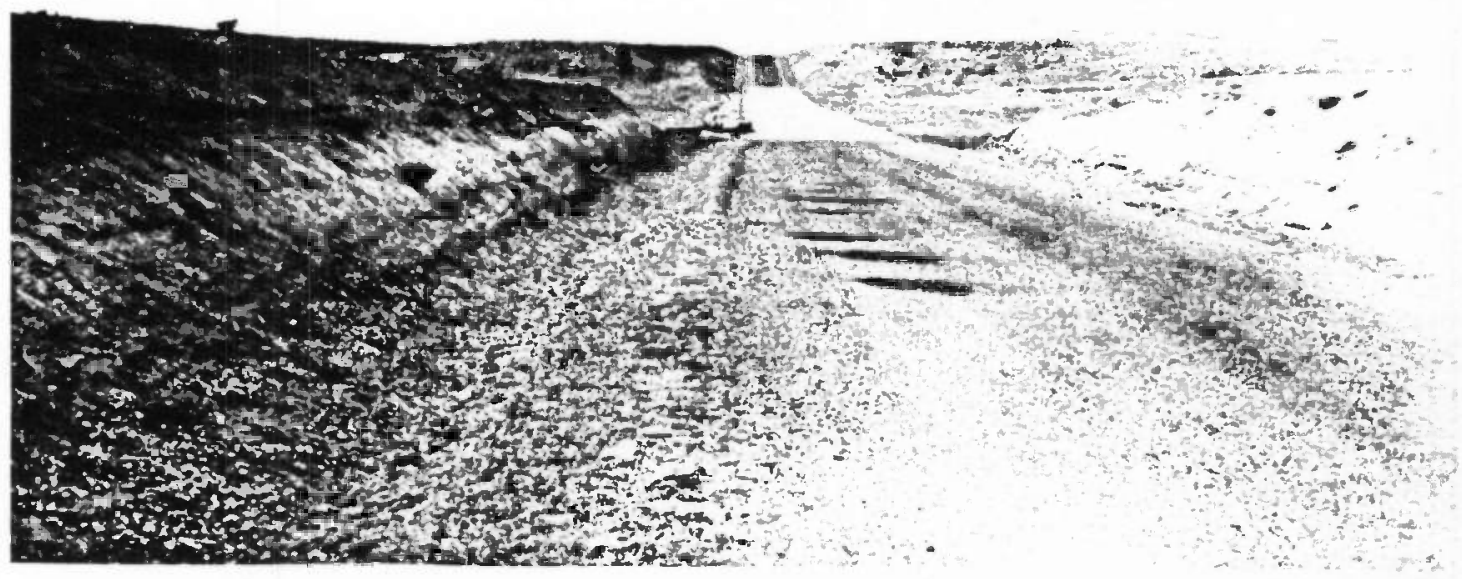




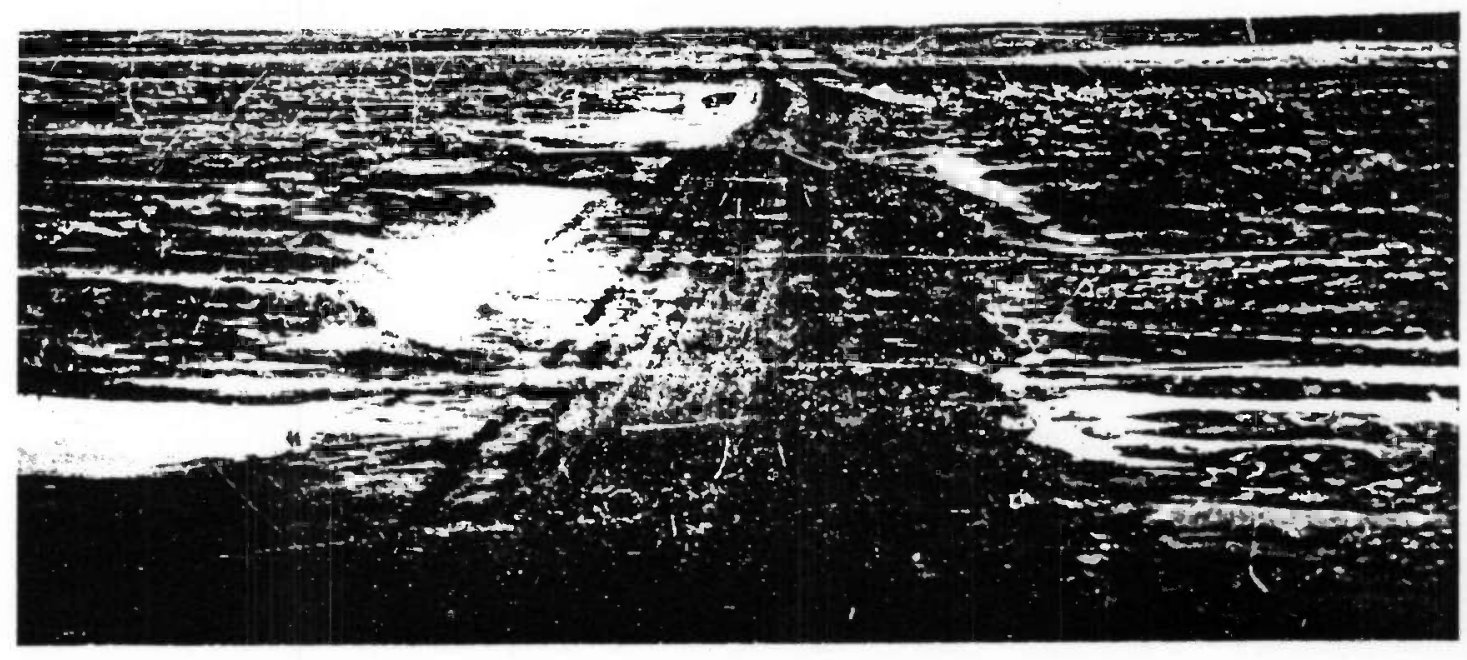

Kilometer station $73+400$

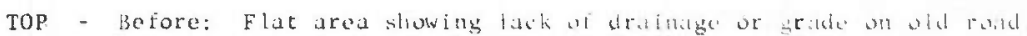

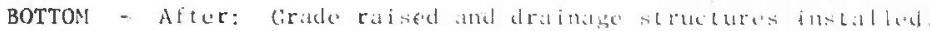

$\checkmark$

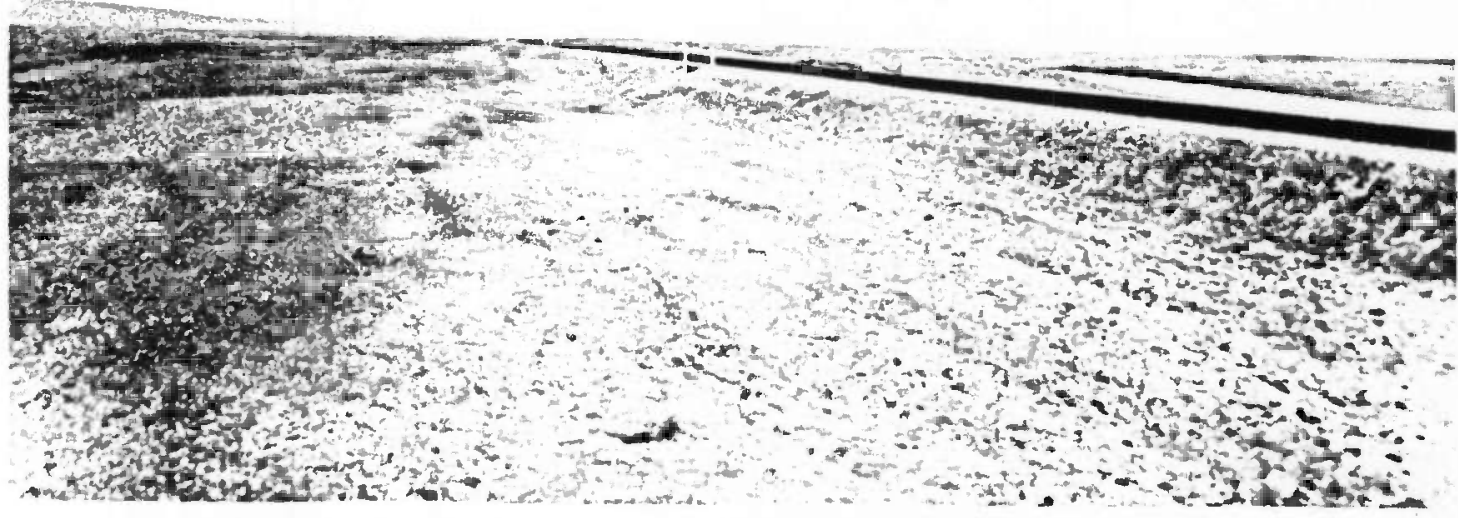




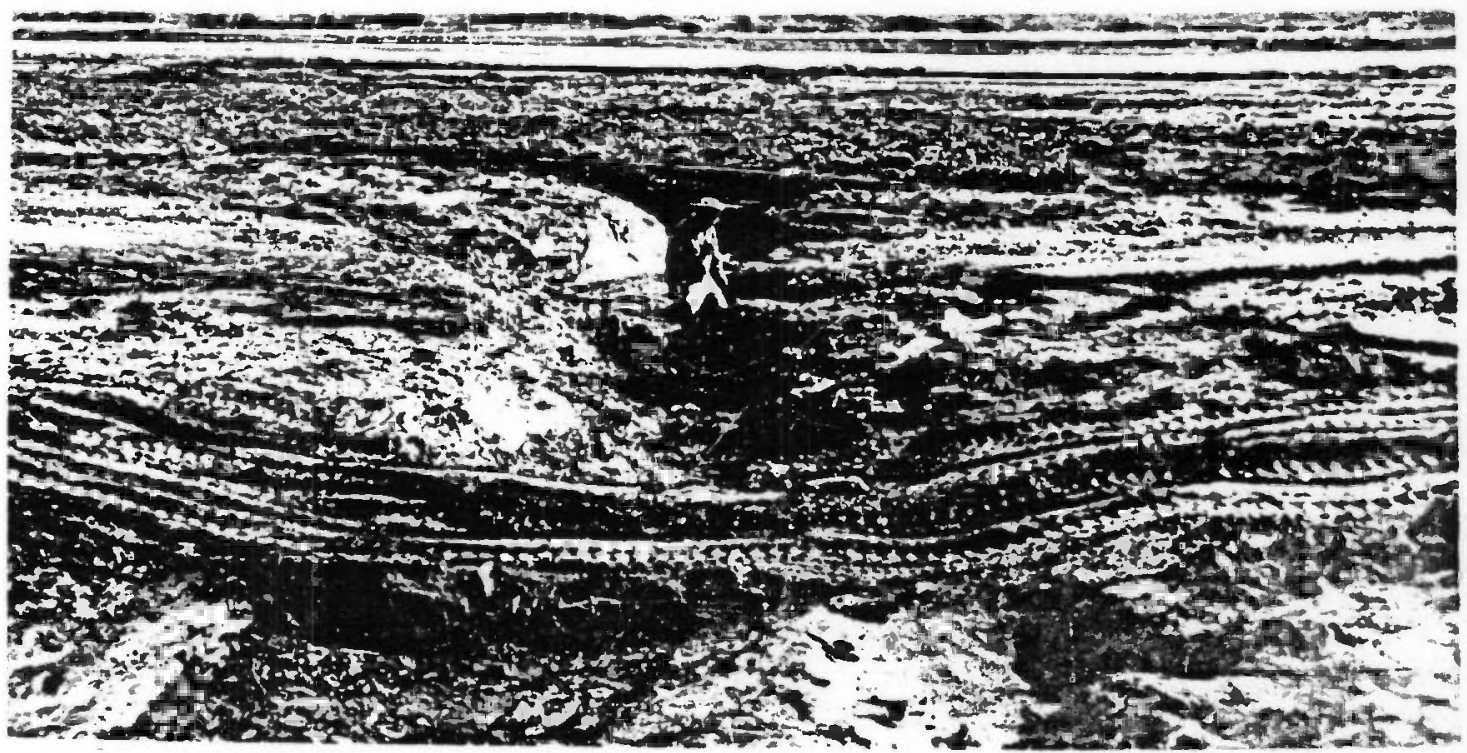

Kilometer Station $62+417$ looking soutl

TOP * Before: Wash out of old road. In Eorescund defour tracks arand old wash out.

BotTon - After: Shows completion of four 54 -inch round Cyp with approbl channel and dike.

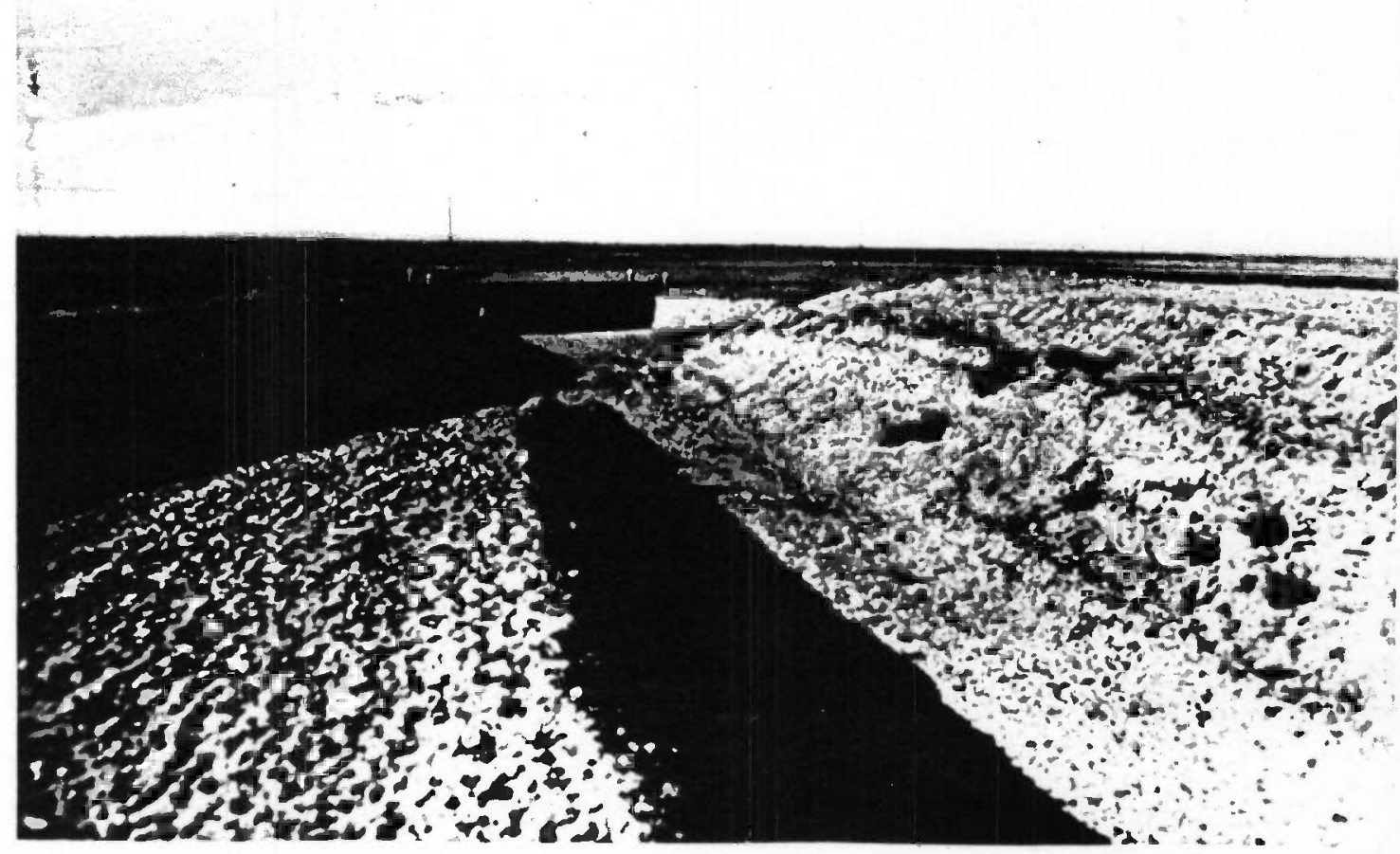




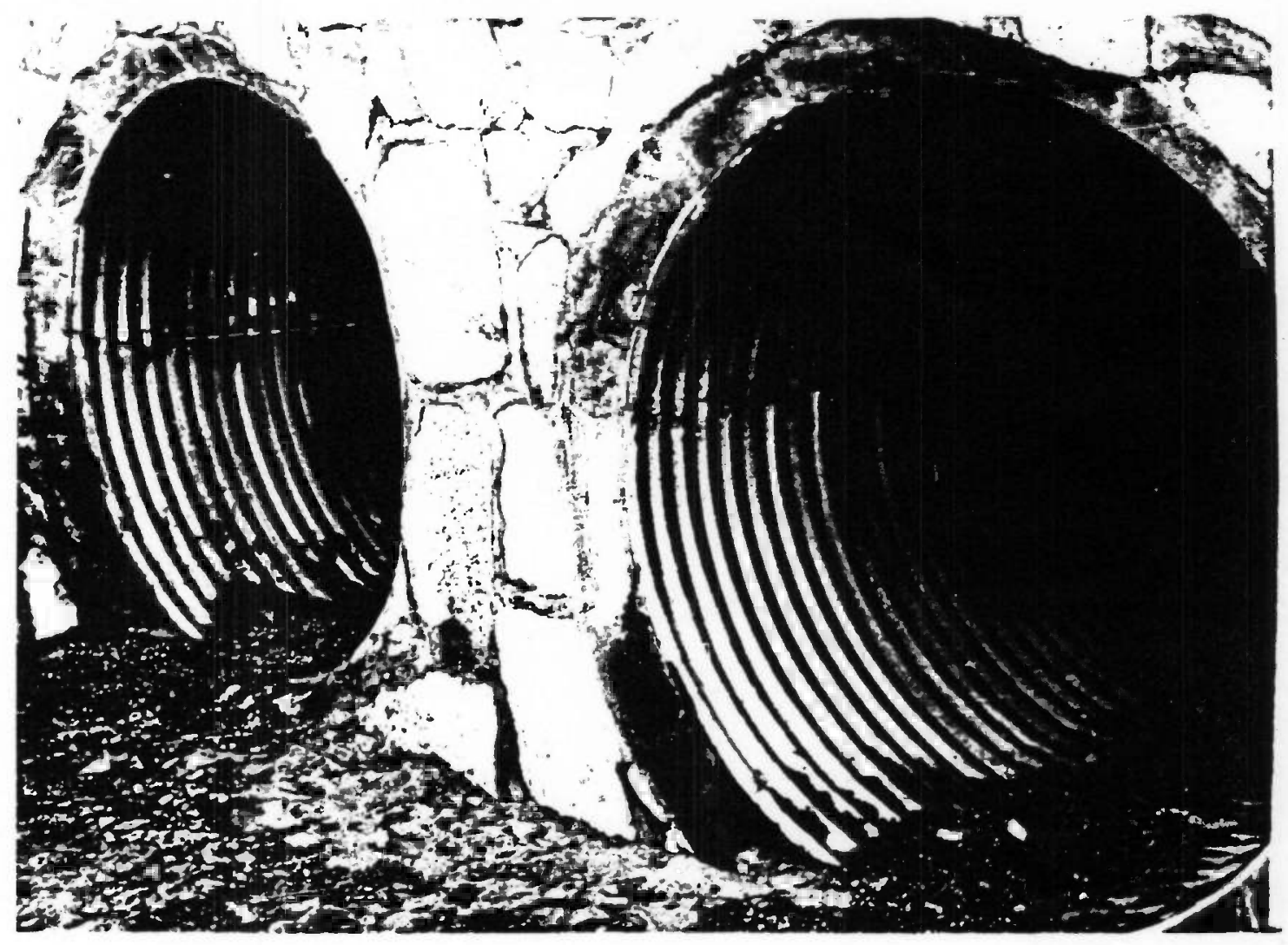

General

Field change. Rounded inlets for CMP culverts to jmprove tlow and increase capacity.
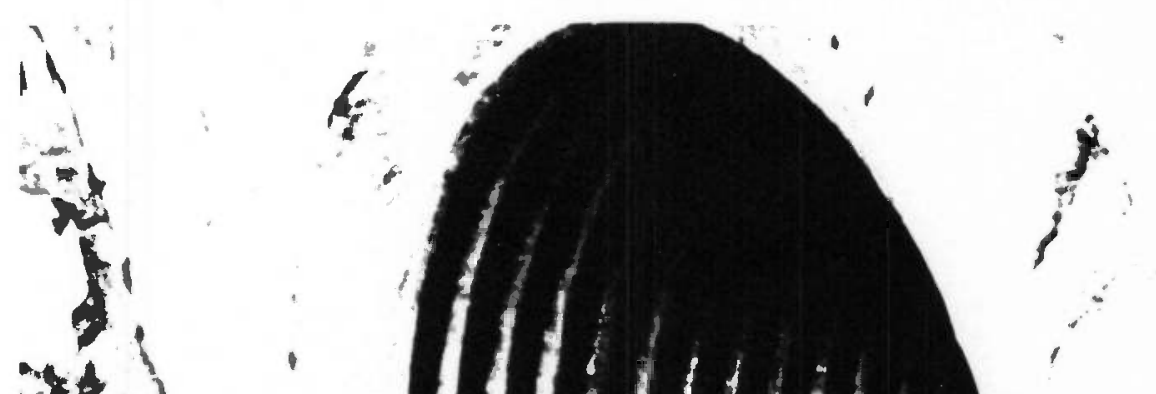

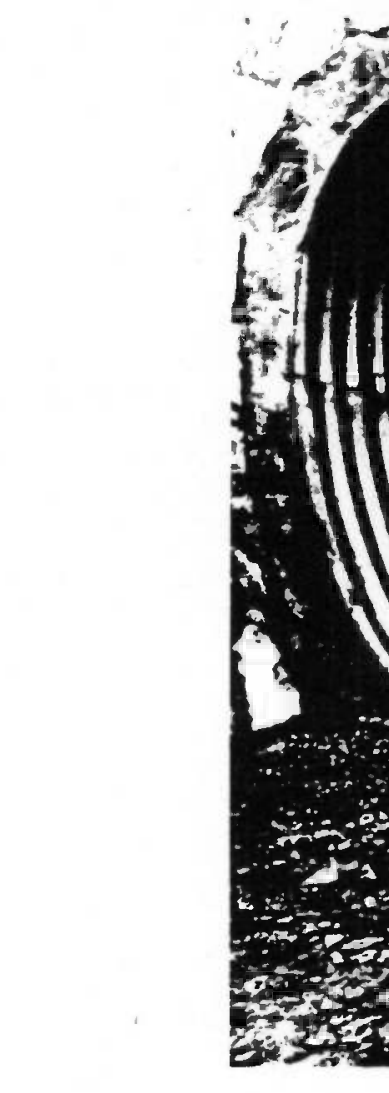

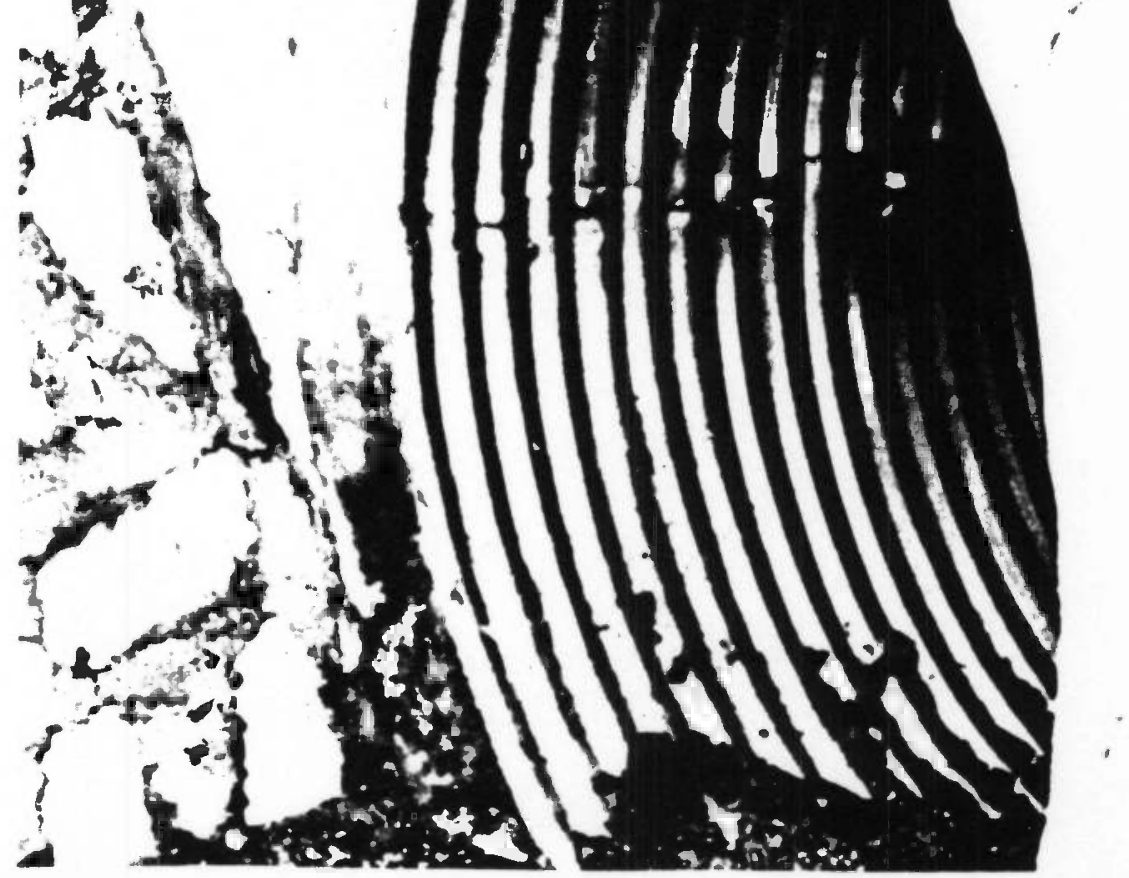




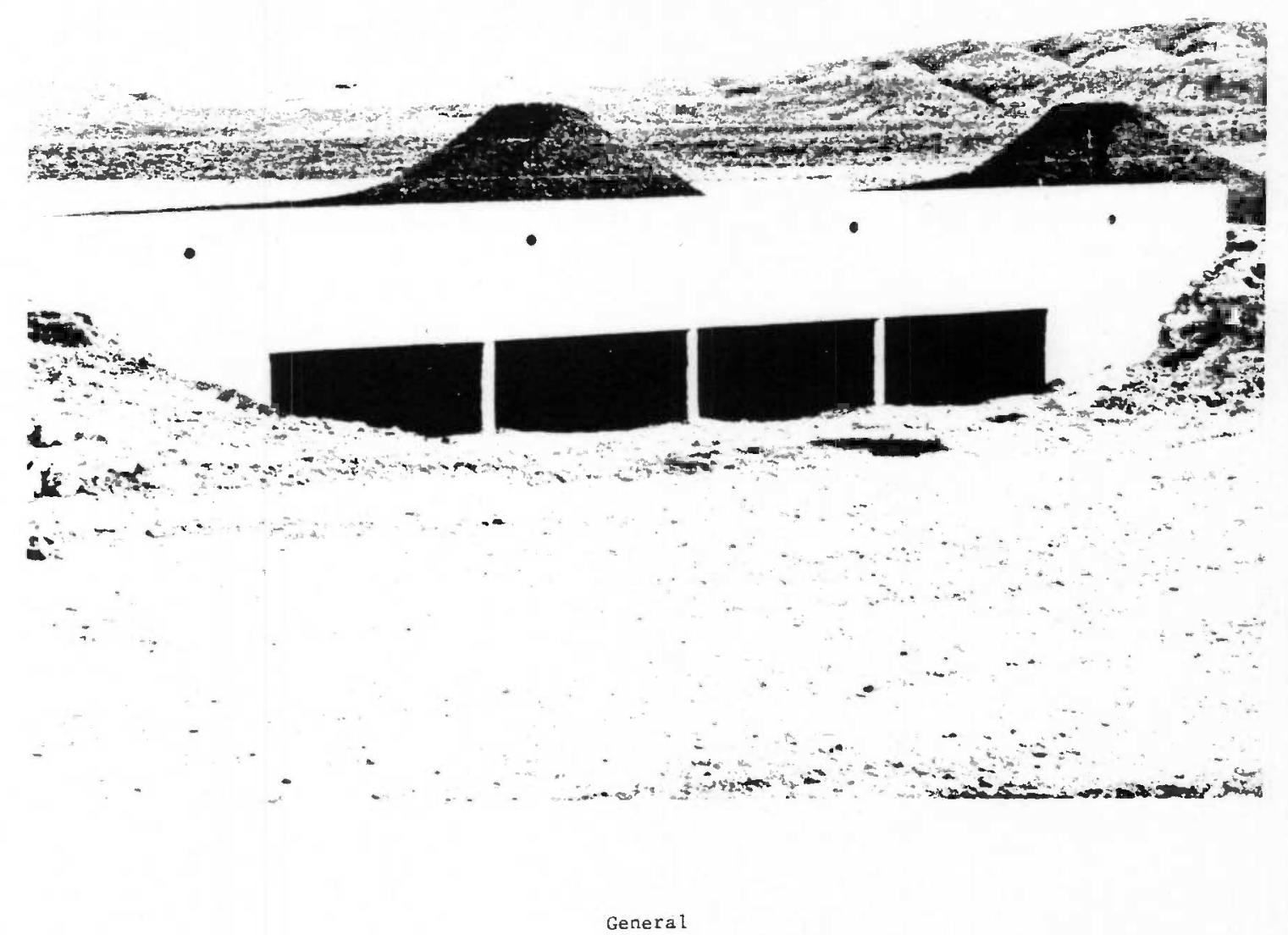

At $46+460,8$ meter by 1 meter box culvert construction nearing completion.

At $51+175$, 6-barrel, 72 -inch CMP culvert being completed.

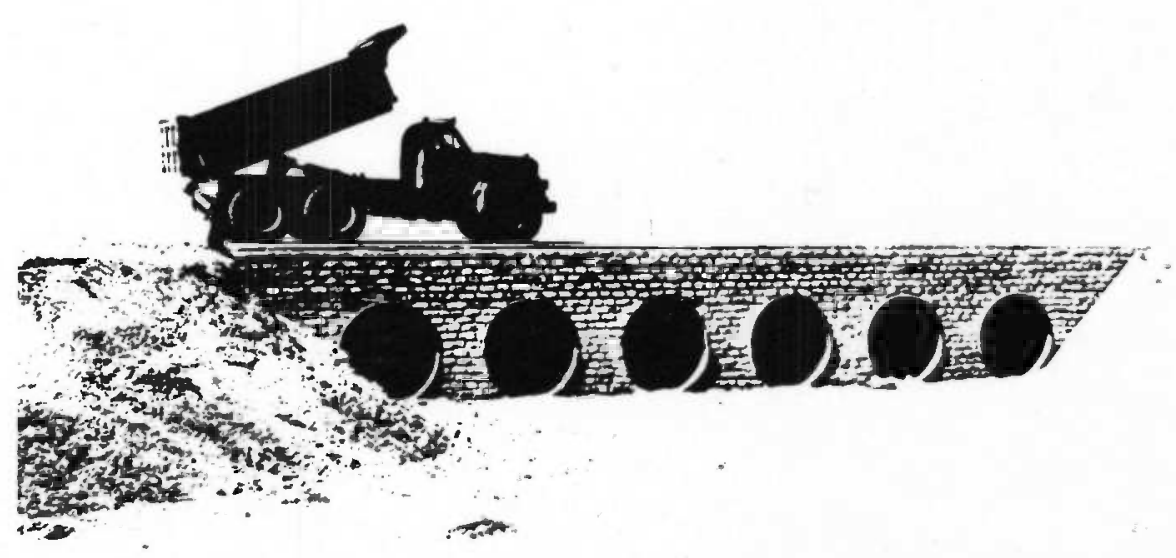




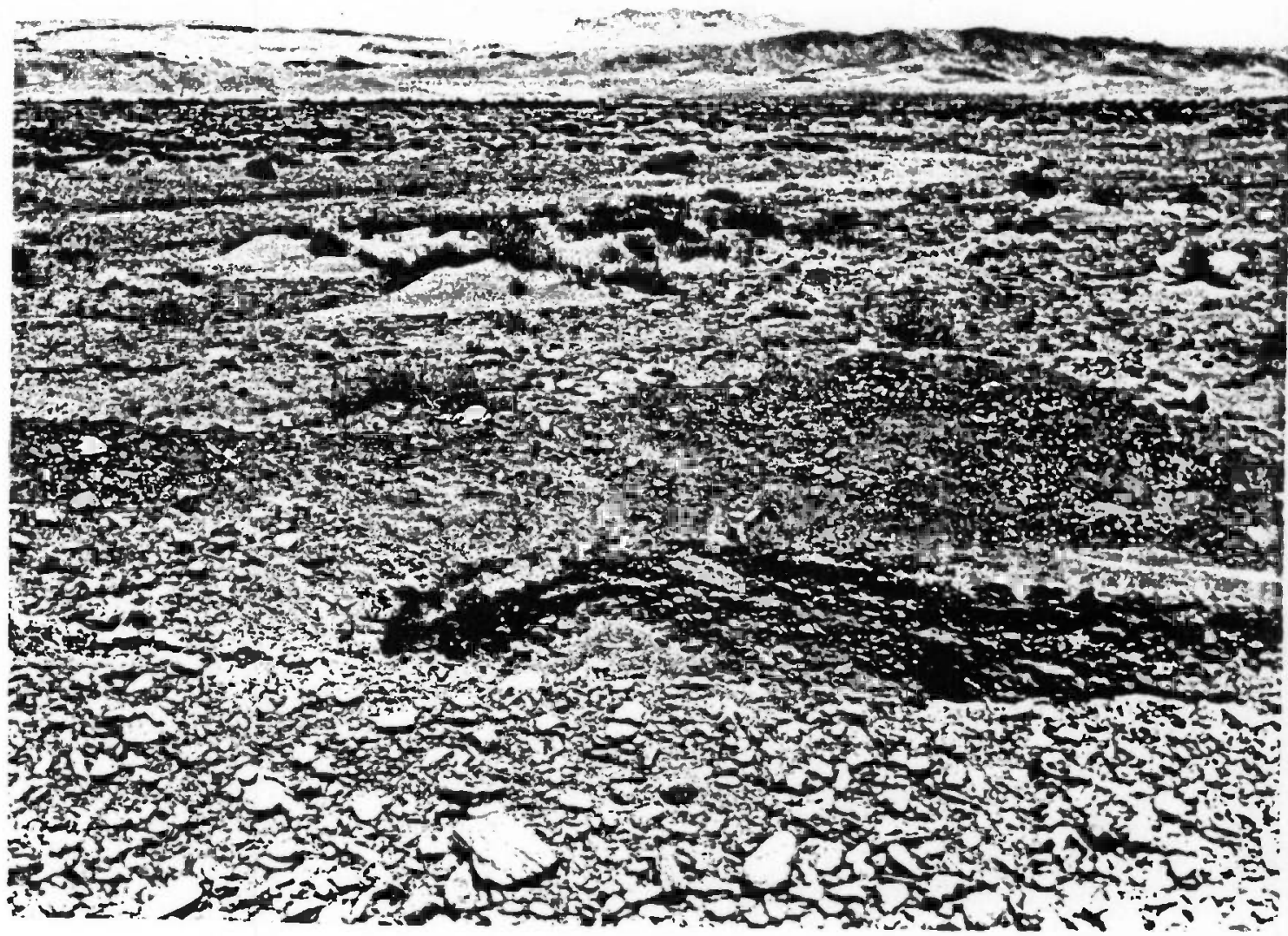

Kilometer Station $34+400$

TOP - Before: Same gravel wash (wadi) approximatelv three kilomuters id

BOTTON - During Construction: Pouring and finishing of concrete in sath a section.

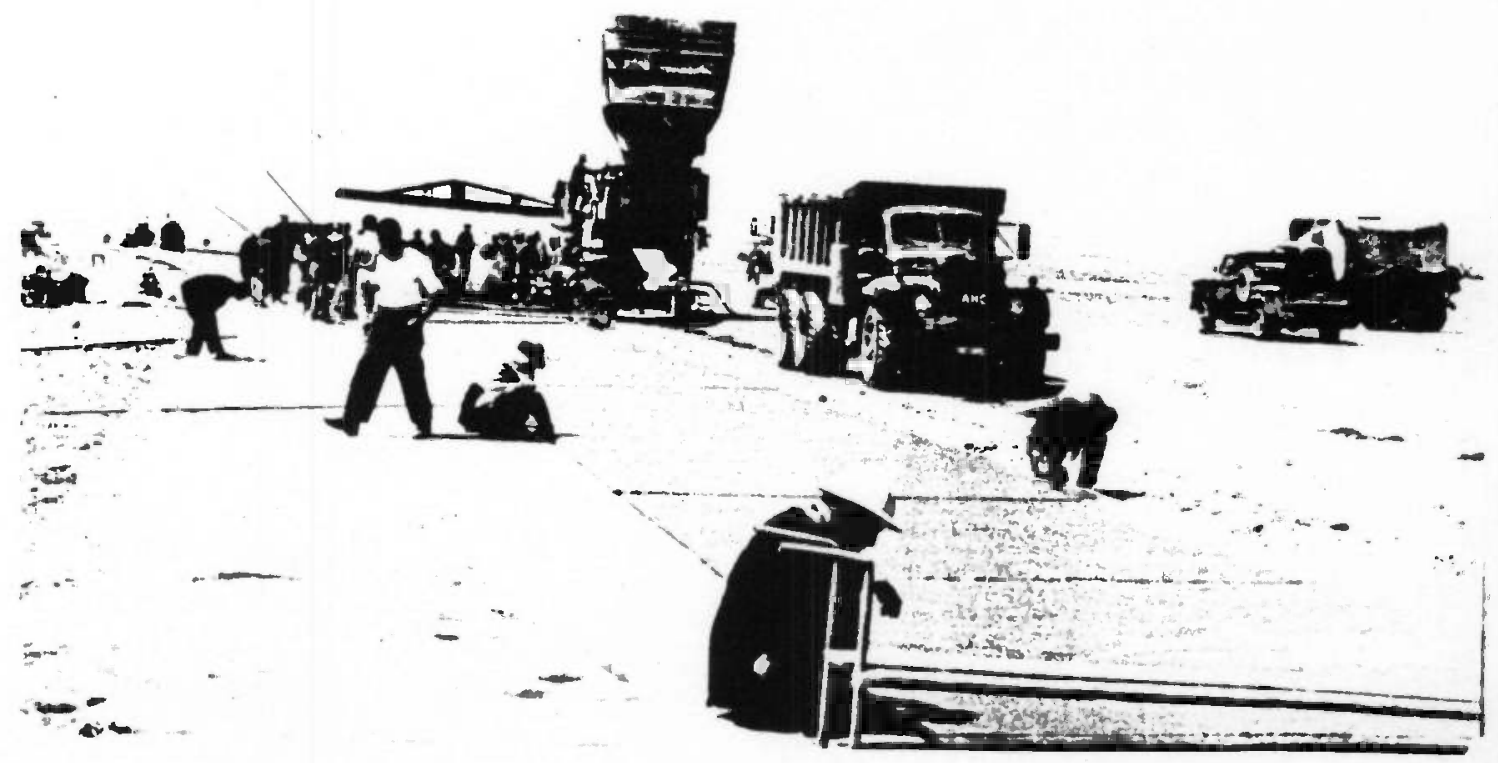




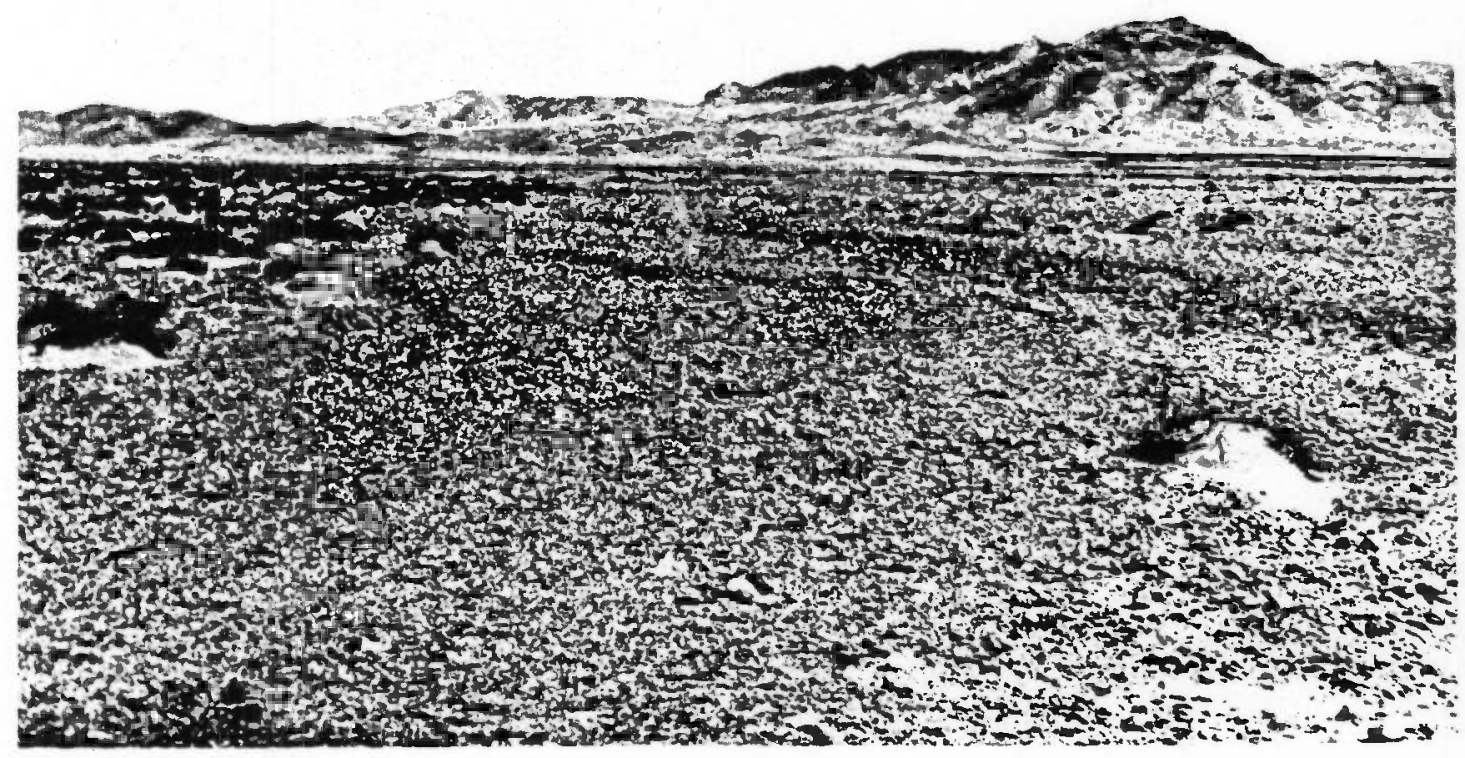

Kilometer station $33+700$

TOP - Before: Gravel wash (wadi) incerstecting road.

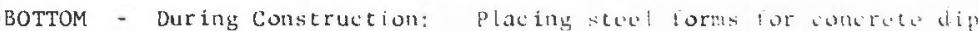
section approximately three ki lometers in tougth.

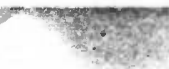

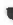

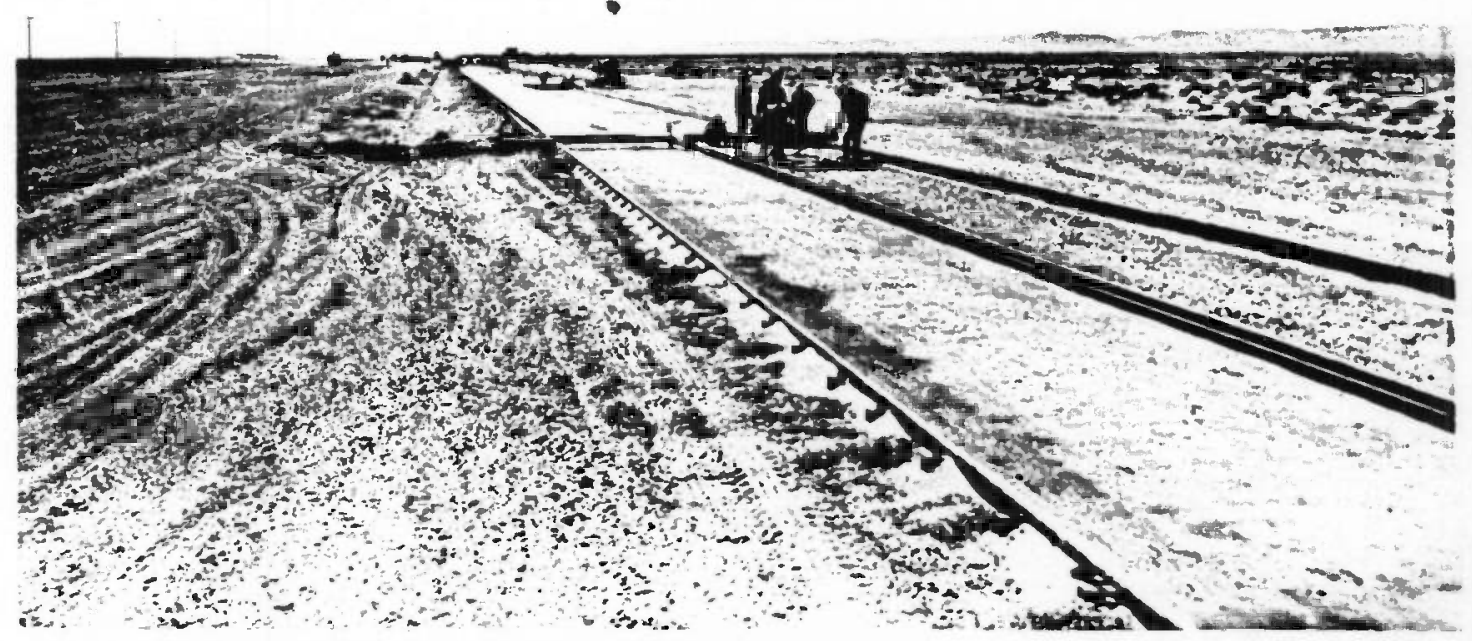




\section{A P P E D I X V}

PROJECT AGREEMENT 
K. EQUIPMENT:

Government-Furnished AHC-HIQ

Heavy Equipment: Prime

Movers (Tractors, Rollers,

Shove 1s, Euc. Dump, Truck

Cranes, Grader, Gradalls,

Loader, Crushers, Mack

Trucks, Trailers, Tankers

and Asphalt Distributor)

Earth Movers

$73 \mathrm{Ea}$

$166 \mathrm{Ea}$

Towed: (Scraper, Euc. Bottom

Dump, Rotary Broom, Conveyors,

Disc Harrow)

$22 \mathrm{Ea}$

$40 \mathrm{Ea}$

Others: (Generators,

Compressors, Clams, Welding

Machines, Concrete Mixers,

Conc. Saws, Forklift, Scale,

Forms)

$54 \mathrm{Ea}$

$52 \mathrm{Ea}$

Light Equipment: (Vehicles

below 1-ton capacity -

Sedans, Carryalls, Pickups

and Ambulance)

$3 \mathrm{Ea}$

$68 \mathrm{Ea}$ 
F. FLEXIBLE PAVEMENT: DBST:

Type A

$98,071.11$ LM

Type B

$5,105.15 \mathrm{LM}$

Type C

$13,973.66 \quad \mathrm{LM}$

$117,149.92 \mathrm{LM}$

G. HAND-PLACED GROUTED RIPRAP:

Culvert Protection

$8,745.37$ SM

Dip Dikes

"J" Sections

Overflow

$6,559.89$ SM

$1,606.12$ SM

396.22 SM

$17,307.60$ SM

H. CONCRETE: REINFORCED AND NON-REINFORCED:

Box Culverts

Foundation \& Protection Culvert

Dips

Bridge

Karese Covers

Guide Posts

Road Sign Posts

Water Level Posts

Kilometer Posts

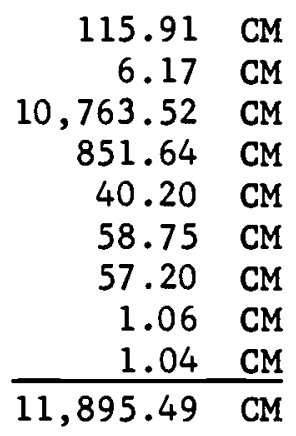

I. POSTS:

Road Signs

\begin{tabular}{rr}
110 & Ea \\
13 & Ea \\
9 & Ea \\
1,174 & Ea \\
\hline 1,306 &
\end{tabular}

J. PERSONNEL (Monthly): (Aug 1966 through Nov 1967)

American

Maximum

USCE

Average

5

5

AHC - HIQ

22

16

Third Country National

USCE

Maximum

Average

6
5

AHC - HIQ

624

5

20

Afghans

USCE

Maximum

24

Average

23

AHC - HIQ

1,446

931 
A. QUANTITY OF EARTHWORK:

Stripping

$152,233 \mathrm{CM}$

Excavation

$756,337 \mathrm{CM}$

Embankment

B . CULVERTS:

Number of Culverts

CMP Culverts

Box Culverts

Length of Culverts

CMP Culverts (Single \& Multiple Barrels)

Box Culverts (Multiples)

$7,270.33$ LF $\frac{64.06 \text { LF }}{7,334.39 \text { LF }}$

Length of CMP Pipes in Place

CMP Culvert

Karese Crossing

$18,292.33 \mathrm{LF}$

$\begin{array}{cc}60 & L F \\ 18,352.33 \quad L F\end{array}$

Equivalent ( 1 meter $\emptyset$ )

$21,126.48 \quad \mathrm{LF}$

C. DIPS:

Length of Dips

Concrete Dip with Shoulder Protection
Concrete Dip without Shoulder Protection
DBST Dip without Shoulder Protection

$4,290 \quad L M$

$380 \quad \mathrm{IM}$

$\begin{array}{ll}1,100 & \mathrm{LM} \\ 5,770 & \mathrm{LM}\end{array}$

D . BRIDGE:

Length of Bridge: 9 Spans

$152.70 \mathrm{LM}$

E. ROADWAY:

Length of Road

Roadway

$117,149.92$ LM

Dips

$5,770.00 \quad$ LM

Bridges

$152.70 \mathrm{LM}$

$123,072.62 \mathrm{LM}$ 
A P P E N D I X IV

TOTAL QUANTITIES OF WORK 


\section{APPENDIX III \\ (TABLE OF LOCATION ANL LENGTH CF)}

BRIDGE ANL LIPS

LOCATICN

LENGTH

TYPE OF STRUCTUKES

BFILGE :

Station $96+472.15-96+624.85$

152.70 Meters Reinforced Concrete

(9 Span)

LIPS :

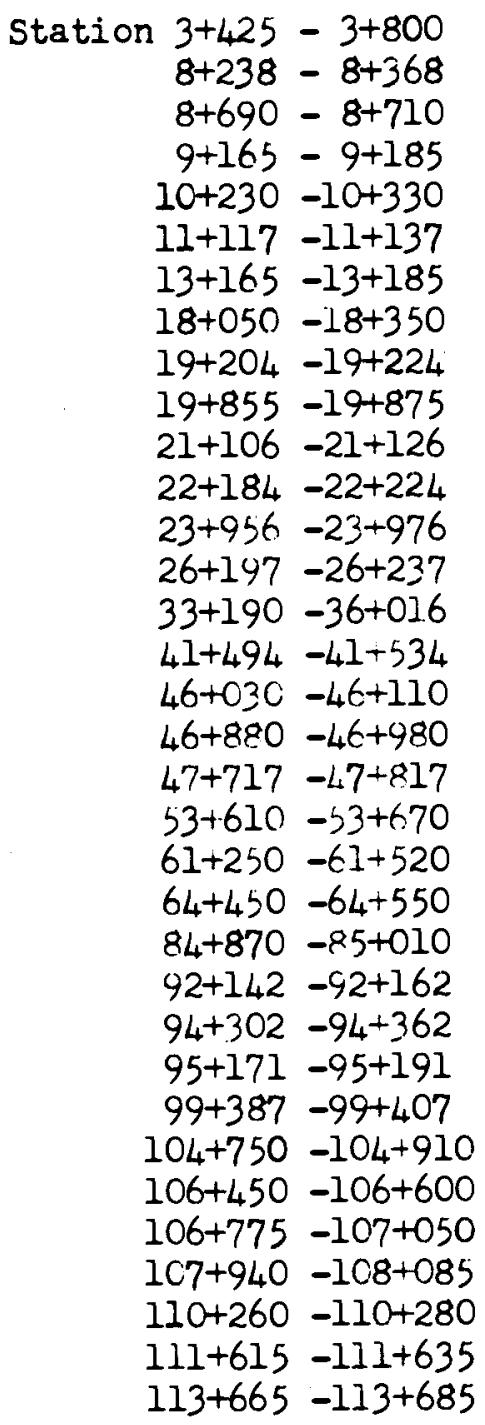

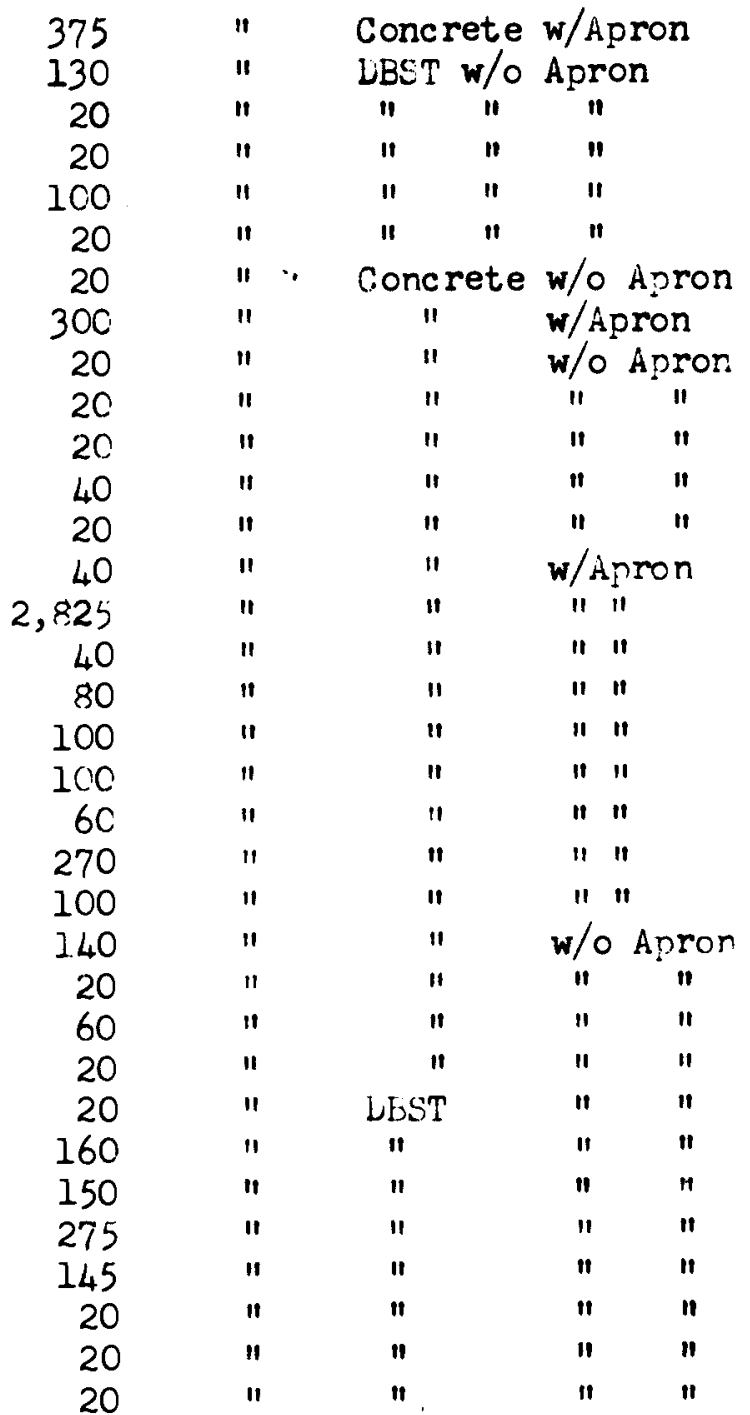


A P P E N D I X III

CULVERT SCHEDULE

PLAN OF DRAINAGE AND DIPS

LOCATION OF BRIDGE AND DRAINAGE DIPS 


\section{APPENDIX III}

\section{CULVERT SCHEDULE}

\begin{tabular}{|c|c|c|c|c|c|c|}
\hline NO. & STATION & SKEW & SIZE & GAGE & LENGTH & REMARKS \\
\hline 1 & $0+229$ & $70^{\circ} \mathrm{L}$ & $1-42^{\prime \prime} \emptyset$ & 12 & $30^{\prime}$ & \\
\hline 2 & $0+725$ & $*$ & $2-48 " 10$ & 12 & $32^{\prime}$ & \\
\hline 3 & $0+841$ & * & $1-5 \mathrm{~N}$ & 12 & $36^{\prime}$ & \\
\hline 4 & $0+883$ & * & $5-5 N$ & 12 & $30^{\prime}$ & \\
\hline 5 & $1+450$ & * & $3-42^{\prime \prime} \emptyset$ & 12 & $28^{\prime}$ & \\
\hline 6 & $2+014$ & * & $1-24^{\prime \prime} \emptyset$ & 14 & $28^{\prime}$ & (Irrig) \\
\hline 7 & $2+268$ & * & $1-5 N$ & 12 & $28^{\prime}$ & (Irrig) \\
\hline 8 & $2+957$ & * & $6-48^{\prime \prime} \emptyset$ & 12 & $28^{\prime}$ & \\
\hline 9 & $3+811$ & $*$ & $1-24^{\prime \prime} 0$ & 14 & $28^{\prime}$ & \\
\hline 10 & $3+951$ & $70^{\circ} \mathrm{L}$ & $1-42 " 0$ & 12 & $30^{\prime}$ & \\
\hline 11 & $4+255$ & $\star$ & $1-7 \mathrm{~N}$ & 12 & $28^{\prime}$ & (Irrig) \\
\hline 12 & $5+308$ & * & $1-7 \mathrm{~N}$ & 12 & $28^{\prime}$ & (Irrig) \\
\hline 13 & $5+480$ & $84^{\circ} \mathrm{L}$ & $1-48^{\prime \prime} \emptyset$ & 12 & $28^{\prime}$ & (Irrig) \\
\hline 14 & $5+550$ & * & $4-48^{\prime \prime} \emptyset$ & 12 & $30^{\prime}$ & \\
\hline 15 & $5+814(19.00 \mathrm{Rt})$ & $11^{\circ}$ & $1-5 N$ & 12 & $28^{\prime}$ & Under T. 0 . \\
\hline 16 & $5+850$ & $\star$ & $1-42^{\prime \prime} \emptyset$ & 12 & $28^{\prime}$ & \\
\hline 17 & $5+973$ & * & $1-5 \mathrm{~N}$ & 12 & $28^{\prime}$ & (Irrig) \\
\hline 18 & $6+087$ & * & $1-5 \mathrm{~N}$ & 12 & $28^{\prime}$ & (Irrig) \\
\hline 19 & $6+227$ & * & $1-5 \mathrm{~N}$ & 12 & $28^{\prime}$ & (Irrig) \\
\hline 20 & $6+340$ & * & $1-42^{\prime \prime} \emptyset$ & 12 & $28^{\prime}$ & $(12+15)$ \\
\hline 21 & $6+774$ & * & $1-42^{\prime \prime} \emptyset$ & 12 & $28^{\prime}$ & \\
\hline 22 & $7+335$ & * & $5-42^{\prime \prime \emptyset}$ & 12 & $30^{\prime}$ & \\
\hline 23 & $7+534$ & $\star$ & $3-9 N$ & 10 & $30^{\prime}$ & \\
\hline 24 & $7+534$ & * & $1-42^{\prime \prime} \emptyset$ & 12 & $30^{\prime}$ & \\
\hline 25 & $8+650$ & $*$ & $1-92^{\prime \prime \emptyset}$ & 12 & $28^{\prime}$ & \\
\hline 26 & $9+010$ & $75^{\circ} \mathrm{I}$ & $1-7 \mathrm{~N}$ & 12 & $30^{\prime}$ & \\
\hline 27 & $9+112$ & $75^{\circ} \mathrm{L}$ & $1-42^{\prime \prime} \emptyset$ & 12 & $30^{\prime}$ & \\
\hline 28 & $9+263$ & * & $2-7 N$ & 12 & $28^{\prime}$ & \\
\hline 29 & $9+471$ & $\star$ & $3-36^{\prime \prime \emptyset}$ & 12 & $28^{\prime}$ & \\
\hline 30 & $9+812.50$ & * & $1-60 " \emptyset$ & 10 & $28^{\prime}$ & \\
\hline 31 & $10+530$ & * & $4-54^{\prime \prime} 0$ & 12 & $28^{\prime}$ & \\
\hline 32 & $10+721$ & $\star$ & $1-60^{\prime \prime} \emptyset$ & 10 & $28^{\prime}$ & \\
\hline 33 & $10+890$ & * & $\begin{array}{l}2-30 " \emptyset \\
9-24^{\prime \prime \emptyset}\end{array}$ & 14 & $28^{\prime}$ & \\
\hline 34 & $11+070$ & * & $2-30 " \emptyset$ & 14 & $28^{\prime}$ & \\
\hline 35 & $11+831$ & $\star$ & $3-8 N$ & 12 & $28^{\prime}$ & \\
\hline 36 & $11+909$ & * & $4-5 N$ & & & \\
\hline & & & $1-6 \mathrm{~N}$ & 12 & $28^{\prime}$ & \\
\hline 37 & $11+985$ & * & $4-30^{\prime \prime \prime}$ & 14 & $28^{\prime}$ & \\
\hline 38 & $12+131$ & * & $6-36^{\prime \prime}$ & 12 & $28^{\prime}$ & \\
\hline 39 & $12+369$ & $*$ & $6-24^{\prime \prime}$ & 14 & $28^{\prime}$ & \\
\hline 40 & $12+975$ & * & $4-30^{\prime \prime}$ & 14 & $28^{\prime}$ & \\
\hline 41 & $13+429$ & * & $5-6 N$ & 12 & $28^{\prime}$ & \\
\hline 42 & $13+918$ & * & $4-30^{\prime \prime} \emptyset$ & 14 & $28^{\prime}$ & \\
\hline
\end{tabular}




\begin{tabular}{|c|c|c|c|c|c|c|}
\hline №. & STATION & SKEW & SIZE & GAGE & LENGTH & REMARKS \\
\hline 43 & $14+435$ & * & $4-48^{\prime \prime} \emptyset$ & 12 & $28^{\prime}$ & \\
\hline 44 & $14+615$ & * & $\begin{array}{l}4-30^{\prime \prime \emptyset} \\
6-24^{\prime \prime} \emptyset\end{array}$ & 14 & $28^{\prime}$ & \\
\hline 45 & $15+118$ & * & $\begin{array}{l}2-30 " \emptyset \\
3-24 " \emptyset\end{array}$ & 14 & $28^{\prime}$ & \\
\hline $\begin{array}{l}46 \\
47 \\
48\end{array}$ & $\begin{array}{l}15+374 \\
16+727 \\
18+455\end{array}$ & $\begin{array}{l}50^{\circ} \mathrm{R} \\
\star \\
\star\end{array}$ & $\begin{array}{l}3-36 " \emptyset \\
5-4 \mathrm{~N} \\
4-36 " \emptyset \\
6-30 " \emptyset\end{array}$ & $\begin{array}{l}12 \\
14 \\
12 \\
14\end{array}$ & $\begin{array}{l}40^{\prime} \\
28^{\prime} \\
30^{\prime}\end{array}$ & \\
\hline 49 & $\begin{array}{l}18+687 \\
18+761.20\end{array}$ & ${ }^{*} 0^{\circ} \mathrm{L}$ & $\begin{array}{l}2-60 " \emptyset \\
1-24 " \emptyset\end{array}$ & $\begin{array}{l}10 \\
14\end{array}$ & $\begin{array}{l}28^{\prime} \\
60^{\prime}\end{array}$ & \\
\hline 50 & $18+926$ & $\star$ & $12-24 " \emptyset$ & 14 & $28^{\prime}$ & \\
\hline 51 & $19+645$ & * & $2-54 " \emptyset$ & 12 & $28^{\prime}$ & \\
\hline 52 & $19+685$ & * & $5-5 N$ & 12 & $30^{\prime}$ & \\
\hline 53 & $20+319$ & * & $3-66 " \emptyset$ & 10 & $28^{\prime}$ & \\
\hline 54 & $20+475$ & * & $4-36 " \emptyset$ & 12 & $28^{\prime}$ & \\
\hline 55 & $20+656$ & * & $3-66 " \emptyset$ & 10 & $28^{\prime}$ & \\
\hline 56 & $21+257$ & * & $5-24^{\prime \prime} \emptyset$ & 14 & $28^{\prime}$ & \\
\hline 57 & $21+333$ & * & $5-24^{\prime \prime} \emptyset$ & 14 & $28^{\prime}$ & \\
\hline 58 & $21+556$ & * & $5-24^{\prime \prime} \emptyset$ & 14 & $28^{\prime}$ & \\
\hline 59 & $21+800$ & * & $\begin{array}{l}4-36^{\prime \prime \emptyset} \\
5-24^{\prime \prime \emptyset}\end{array}$ & $\begin{array}{l}12 . \\
14\end{array}$ & $\begin{array}{l}28^{\prime} \\
28^{\prime}\end{array}$ & \\
\hline 60 & $22+475$ & * & $\begin{array}{l}4-36^{\prime \prime} \\
9-24^{\prime \prime}\end{array}$ & $\begin{array}{l}12 \\
14\end{array}$ & $28^{\prime}$ & \\
\hline 61 & $23+405$ & * & $\begin{array}{l}3-8 \mathrm{~N} \\
3-54^{\prime \prime} \emptyset\end{array}$ & $\begin{array}{l}14 \\
12 \\
12\end{array}$ & $30^{\prime}$ & \\
\hline 62 & $24+000$ & * & $4-30 " \emptyset$ & $\begin{array}{l}12 \\
14\end{array}$ & $28^{\prime}$ & \\
\hline 63 & $24+288$ & * & $2-8 N$ & 12 & $30^{\prime}$ & \\
\hline 64 & $24+959$ & * & $3-10 N$ & $10 \& 8$ & $28^{\prime}$ & \\
\hline 65 & $25+233$ & * & $4-66^{\prime \prime} \emptyset$ & 10 & $28^{\prime}$ & \\
\hline 66 & $25+402$ & * & $3-48^{\prime \prime} \emptyset$ & 12 & $28^{\prime}$ & \\
\hline 67 & $25+571$ & $*$ & $5-60^{\prime \prime} \emptyset$ & 10 & $28^{\prime}$ & \\
\hline 68 & $25+940$ & $80^{\circ} \mathrm{L}$ & $4-54^{\prime \prime \emptyset}$ & 12 & $34^{\prime}$ & \\
\hline 69 & $26+400$ & & $1-24^{\prime \prime} \emptyset$ & 14 & $28^{\prime}$ & \\
\hline 70 & $26+523$ & $71^{\circ} \mathrm{L}$ & $1-24^{\prime \prime} \emptyset$ & 14 & $30^{\prime}$ & \\
\hline 71 & $26+598$ & * & $1-24^{\prime \prime} \emptyset$ & 14 & $28^{\prime}$ & \\
\hline 72 & $27+000$ & * & $3-8 N$ & 12 & $30^{\prime}$ & \\
\hline 73 & $27+325$ & * & $5-36^{\prime \prime} \emptyset$ & 12 & $28^{\prime}$ & \\
\hline 74 & $27+513$ & $64^{\circ} \mathrm{R}$ & $4-30^{\prime \prime} \emptyset$ & $\begin{array}{l}12 \\
14\end{array}$ & $32^{\prime}$ & \\
\hline 75 & $28+586$ & $67^{\circ} \mathrm{R}$ & $6-36^{\prime \prime} \emptyset$ & $\begin{array}{l}14 \\
12\end{array}$ & 321 & \\
\hline 76 & $29+040$ & $65^{\circ} \mathrm{R}$ & $7-4 N$ & 14 & & \\
\hline & & & $3-30^{\prime \prime} \emptyset$ & 14 & $32^{\prime}$ & \\
\hline 77 & $29+700$ & * & $1-24^{\prime \prime} \emptyset$ & 14 & $28^{\prime}$ & (Irrig) \\
\hline 78 & $29+702.75$ & - & $3-24^{\prime \prime} \emptyset$ & 14 & $22^{\prime}$ & Under T.O.) \\
\hline 79 & $30+275$ & $85^{\circ} \mathrm{R}$ & $4-36^{\prime \prime \emptyset}$ & 12 & $28^{\prime}$ & \\
\hline 80 & $31+212$ & $70^{\circ} \mathrm{L}$ & $4-30^{\prime \prime} \emptyset$ & 14 & $30^{\prime}$ & \\
\hline 81 & $31+525$ & * & $3-36^{\prime \prime} \emptyset$ & 12 & $28^{\prime}$ & \\
\hline 82 & $36+375$ & * & $2-30^{\prime \prime} \emptyset$ & 14 & $30^{\prime}$ & \\
\hline
\end{tabular}




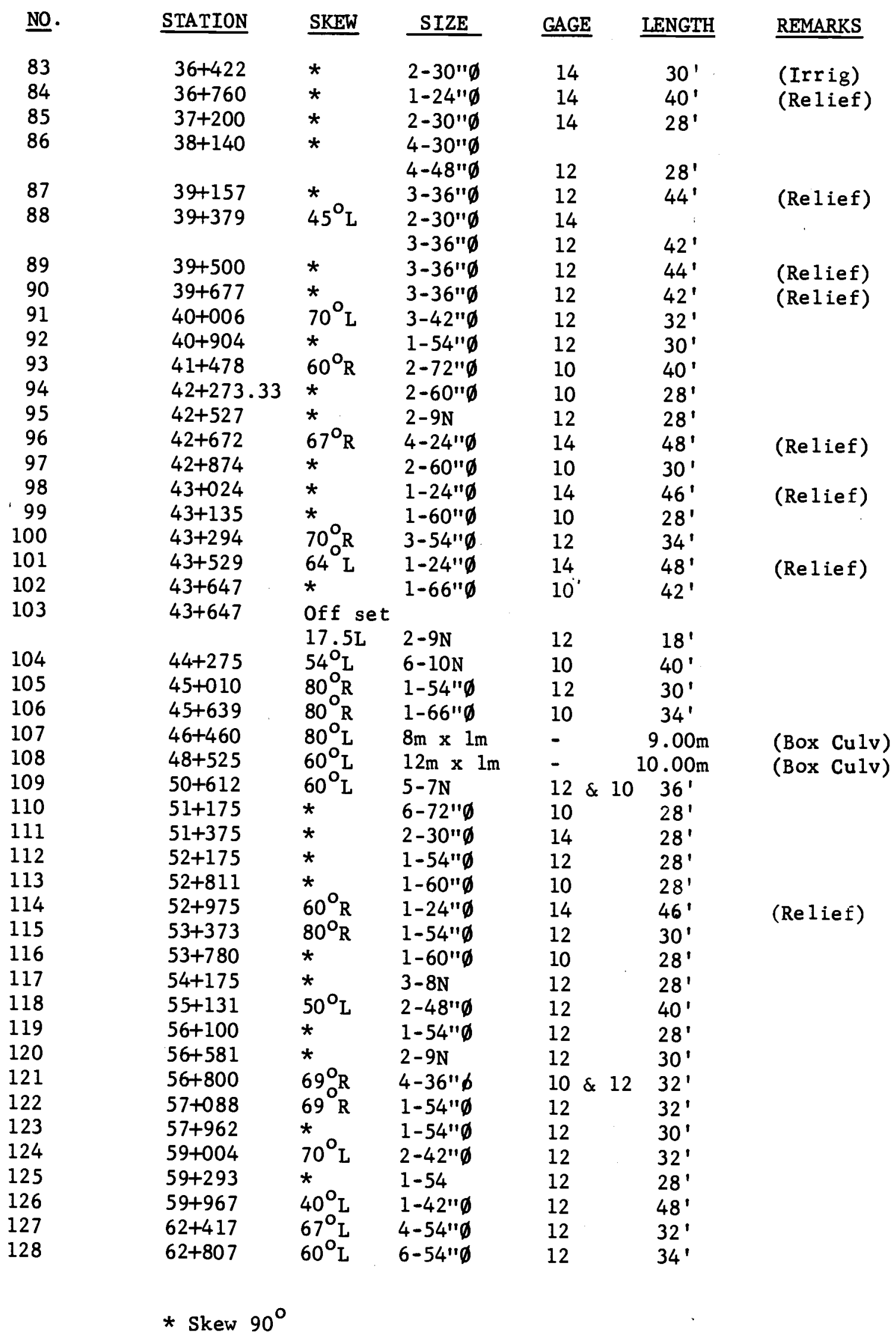




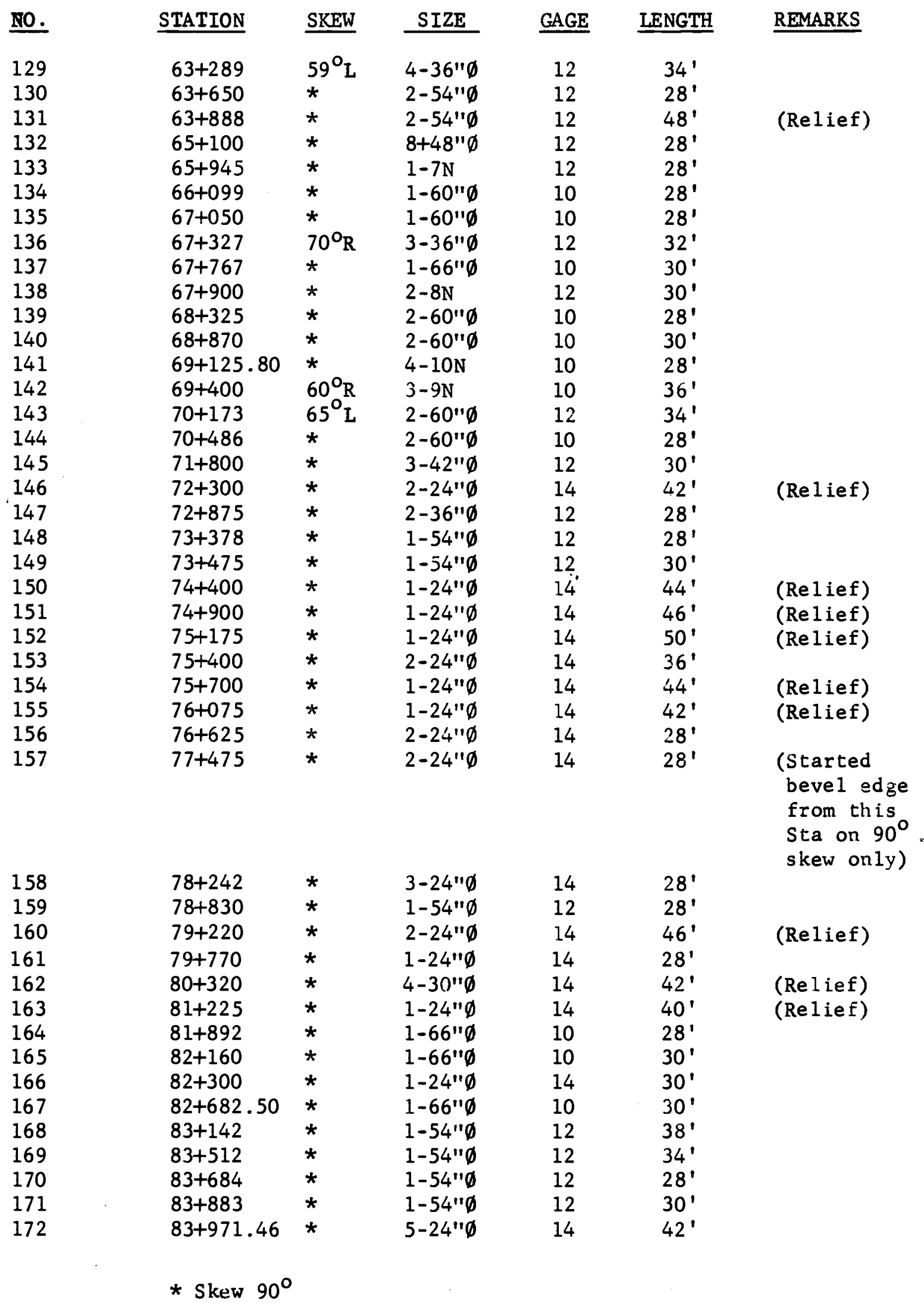




\begin{tabular}{|c|c|c|c|c|c|c|}
\hline No. & STATION & SKEW & SIZE & GAGE & LENGTH & REMARKS \\
\hline 173 & $84+377$ & * & $1-66^{\prime \prime \emptyset}$ & 10 & $48^{\prime}$ & \\
\hline 174 & $84+723$ & * & $1-54^{\prime \prime} \emptyset$ & 12 & $28^{\prime}$ & \\
\hline 175 & $85+725$ & * & $3-30 " \emptyset$ & 14 & $28^{\prime}$ & \\
\hline 176 & $85+930$ & * & $1-24^{\prime \prime} \emptyset$ & 14 & $48^{\prime}$ & (Relief) \\
\hline 177 & $86+800$ & * & $1-72^{\prime \prime} \emptyset$ & 10 & $48^{\prime}$ & \\
\hline 178 & $87+203$ & $60^{\circ} \mathrm{R}$ & $1-54^{\prime \prime \emptyset}$ & 12 & $44^{\prime}$ & \\
\hline 179 & $88+075$ & $\star$ & $5-24^{\prime \prime} \emptyset$ & 14 & $32 '$ & \\
\hline 180 & $89+576$ & $\star$ & $1-60^{\prime \prime} \emptyset$ & 10 & $28^{\prime}$ & \\
\hline 181 & $89+774$ & $*$ & $5-8 N$ & $12 \& 10$ & $32^{\prime}$ & \\
\hline 182 & $89+983$ & $58^{\circ} \mathrm{R}$ & $1-24 " \emptyset$ & 14 & $42^{\prime}$ & $\begin{array}{l}\text { (Left Relief) } \\
\text { (Right Std Hdwl) }\end{array}$ \\
\hline 183 & $90+539$ & $54^{\circ} \mathrm{R}$ & $1-60^{\prime \prime} \emptyset$ & 10 & $36^{\prime}$ & \\
\hline 184 & $90+688$ & $69^{\circ} \mathrm{L}$ & $1-54^{\prime \prime \emptyset}$ & 12 & $32^{\prime}$ & \\
\hline 185 & $90+832.87$ & $40^{\circ} \mathrm{R}$ & $2-72 " \emptyset$ & 10 & $58^{\prime}$ & \\
\hline 186 & $91+298$ & $\star$ & $1-24^{\prime \prime} \emptyset$ & 14 & $28^{\prime}$ & \\
\hline 187 & $91+641$ & * & $2-54^{\prime \prime} \emptyset$ & 12 & $28^{\prime}$ & \\
\hline 188 & $92+253$ & $68^{\circ} \mathrm{R}$ & $2-24^{\prime \prime} \emptyset$ & 14 & $34^{\prime}$ & \\
\hline 189 & $92+371$ & $55^{\circ} \mathrm{R}$ & $1-66^{\prime \prime \emptyset}$ & 10 & $40^{\prime}$ & \\
\hline 190 & $92+900$ & * & $2-72^{\prime \prime} \emptyset$ & 10 & $40^{\prime}$ & \\
\hline 191 & $93+089$ & * & $1-60^{\prime \prime} \emptyset$ & 10 & $42^{\prime}$ & \\
\hline 192 & $93+362$ & * & $2-66^{\prime \prime} \emptyset$ & 10 & $34^{\prime}$ & \\
\hline 193 & $93+490$ & $56^{\circ} \mathrm{L}$ & $1-66^{\prime \prime} \emptyset$ & $10^{\prime}$ & $44^{\prime}$ & \\
\hline 194 & $93+630$ & $50^{\circ} \mathrm{R}$ & $1-24^{\prime \prime} \emptyset$ & 14 & $66^{\prime}$ & \\
\hline 195 & $93+798$ & $70^{\circ} \mathrm{L}$ & $1-54^{\prime \prime} \emptyset$ & 12 & $34^{\prime}$ & \\
\hline 196 & $94+016$ & $\star$ & $1-66^{\prime \prime} \emptyset$ & 10 & $30^{\prime}$ & \\
\hline 197 & $94+190$ & * & $1-66^{\prime \prime} \emptyset$ & 10 & $30^{\prime}$ & \\
\hline 198 & $94+742$ & * & $1-54^{\prime \prime} \emptyset$ & 12 & $28^{\prime}$ & \\
\hline 199 & $95+100$ & * & $1-24^{\prime \prime} \emptyset$ & 14 & $30^{\prime}$ & \\
\hline 200 & $95+500$ & $\star$ & $3-24^{\prime \prime}$ & 14 & $28^{\prime}$ & \\
\hline 201 & $.95+016.50$ & off set & & & & \\
\hline & & $9.00 \mathrm{~m}$ & $1-24^{\prime \prime} \emptyset$ & 14 & $32^{\prime}$ & (Under T.O.) \\
\hline 202 & $96+025$ & $65^{\circ} \mathrm{R}$ & $1-54 " \emptyset$ & 12 & $34^{\prime}$ & (Irrig) \\
\hline 203 & $96+374.50$ & $52^{\circ} \mathrm{R}$ & $3-24^{\prime \prime} \emptyset$ & 14 & $50^{\prime}$ & \\
\hline 204 & $97+045$ & $\star$ & $1-66^{\prime \prime} \emptyset$ & 10 & $44^{\prime}$ & \\
\hline 205 & $97+344$ & $78^{\circ} \mathrm{L}$ & $2-72 " \emptyset$ & 10 & $64^{\prime}$ & \\
\hline 206 & $98+025$ & $*$ & $2-66 " \emptyset$ & 10 & $66^{\prime}$ & \\
\hline 207 & $100+047.50$ & * & $1-60 " \emptyset$ & 8 & $28^{\prime}$ & \\
\hline 208 & $100+611$ & * & $2-60^{\prime \prime \emptyset}$ & 8 & $28^{\prime}$ & \\
\hline 209 & $100+907$ & * & $2-66 " \emptyset$ & 10 & $28^{\prime}$ & \\
\hline 210 & $101+469$ & * & $2-60^{\prime \prime \emptyset}$ & 8 & $28^{\prime}$ & \\
\hline 211 & $102+155$ & * & $2-66 " \emptyset$ & 10 & $34^{\prime}$ & \\
\hline 212 & $102+702.50$ & * & $1-144^{\prime \prime} \emptyset$ & 8 & $64^{\prime}-4^{\prime \prime}$ & (M.P.P.) \\
\hline 213 & $102+980$ & * & $1-60^{\prime \prime} \emptyset$ & 8 & 28 ' & \\
\hline 214 & $103+569$ & * & $1-60^{\prime \prime} \emptyset$ & 8 & $28^{\prime}$ & \\
\hline 215 & $104+168$ & * & $1-60^{\prime \prime} \emptyset$ & 8 & $30^{\prime}$ & \\
\hline 216 & $105+601$ & $70^{\circ} \mathrm{R}$ & $2-66^{\prime \prime} \emptyset$ & 10 & $38^{\prime}$ & \\
\hline 217 & $106+114$ & $\star$ & $1-60 " \emptyset$ & 8 & $28^{\prime}$ & \\
\hline 218 & $109+369$ & $\star$ & $1-66^{\prime \prime} \emptyset$ & 10 & $42^{\prime}$ & \\
\hline 219 & $110+269.25$ & * & $1-6 N$ & 12 & $34^{\circ}$ & \\
\hline 220 & $112+912$ & $65^{\circ} \mathrm{L}$ & $4-24^{\prime \prime \emptyset}$ & 14 & $32^{\prime}$ & \\
\hline
\end{tabular}

* Skew $90^{\circ}$ 


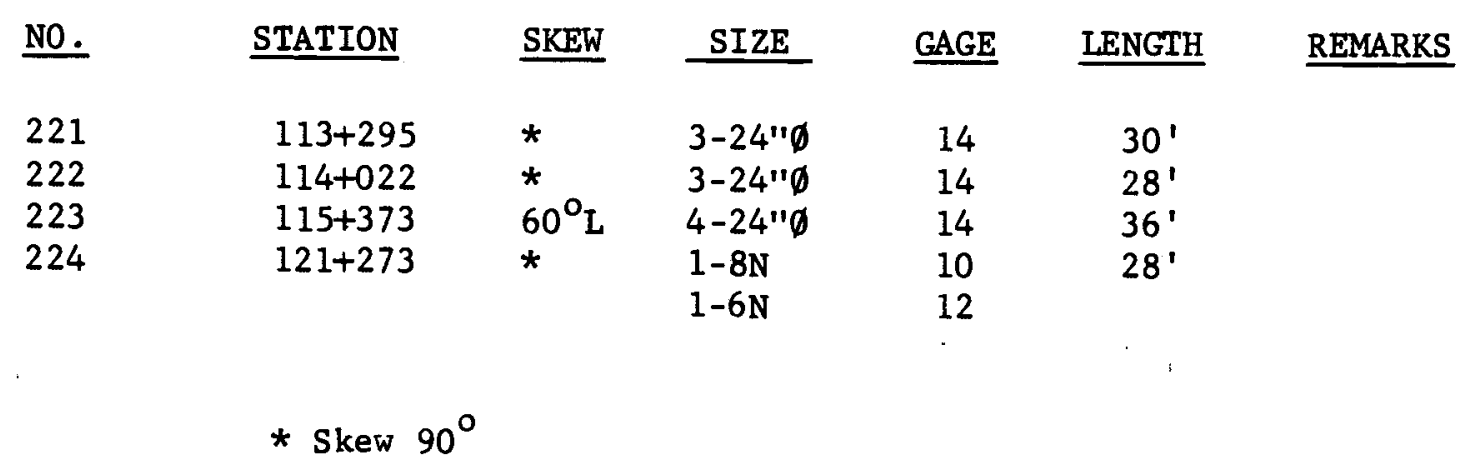




\section{APPENDIX II}

\section{MATER IALS}

I. STABILIZED AGGREGATE BASE COURSE:

SIEVE DESIGNATION

$$
\begin{aligned}
& \begin{array}{l}
1 " \\
\text { 1" }
\end{array} \\
& 3 / 8^{\prime \prime} \\
& \text { No. } 4 \\
& \text { No. } 10 \\
& \text { No. } 40 \\
& \text { No. } 200
\end{aligned}
$$

PERCENT BY WT PASSING SQ MESH

$$
\begin{aligned}
100 \\
70-100 \\
50-80 \\
35-65 \\
25-50 \\
10-30 \\
0-8
\end{aligned}
$$

The portion of any component or of the completed course passing the No. 40 sieve shall be either non-plastic or shall have a Liquid Limit not greater than 25 and a Plasticity Index not greater than 5 .

II. SELECT BORROW MATERIAL COURSE:

SIEVE SIZE

3" SQ.

No. 10

No. 200
PERCENT PASSING

100

75

25

Portion of material passing the No. 40 sieve shall have a Liquid Limit less than 35 and Plasticity Index less than 12. Where material is required to be nonfrost susceptible, $0.02 \mathrm{MM}$ particles will not exceed 3 percent by weight of total sample.

III. SUBBASE COURSE AND FILTER COURSE:

SIEVE SIZE

$3 "$
$3 "$
$3 / 8 "$
No. 4
No. 10
No. 40
No. 200

PERCENT PASSING BY WT SQ MESS SIEVE

SUBBASE

F ILTER

100
$65-100$
$40-95$
$27-79$
$15-60$
$0-32$
$0-8$

Liquid Limit - Not greater than 25.

Plasticity Index - Not greater than 5 .

Less than $0.02 \mathrm{M}$ particles - Not to exceed 3 percent. 


\section{GRADATION OF CONCRETE AGGREGATES - PAVEMENT}

I. COARSE: MAXIMUM NOMINAL SIZE $1 \frac{1}{2}$ inches

SIEVE SIZE (US Std D Mesh)

PERCENT PASSING

No. 4 to $3 / 4^{\prime \prime}, 3 / 4^{\prime \prime}$ to $1 \frac{1}{2} "$

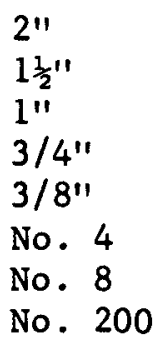

100

$90-100$

100

$20-55$

$90-100$

$20-55$

$0-10$

$0-5$

$0-1$

II. FINE:

CUMULATIVE PERCENT BY WT

SIEVE SIZE (US Std D Mesh)

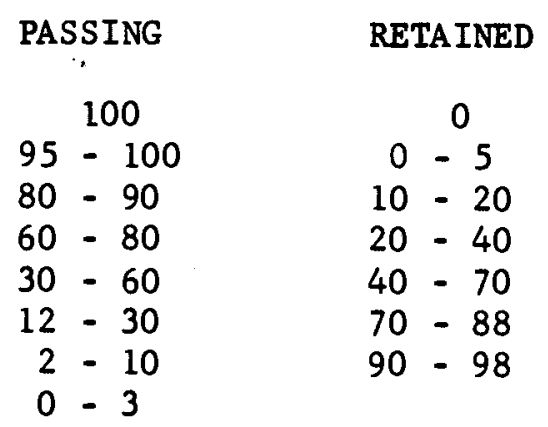

Fineness Modulus not less than 2.40 nor more than 2.90 . Also for use on Grouted Hand-Placed Riprap. 
GRADATION OF CONCRETE AGGREGATES - STRUCTURES

I. COARSE:

\section{PERCENT PASSING}

SIEVE SIZE (US Std D Mesh)

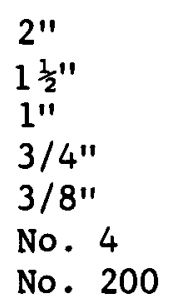

No. 4 to $3 / 4^{\prime \prime} 3 / 4^{\prime \prime}$ to $1 \frac{1}{2} "$

$$
\begin{array}{rlrl} 
& & 100 \\
100 & 90 & -100 \\
95 & -100 & 20 & -45 \\
30 & -55 & 0 & -10 \\
0 & -5 & 0 & -5 \\
0 & -1 & &
\end{array}
$$

II. FINE:

PERCENT PASSING

$$
\begin{aligned}
& 3 / 8 " \\
& \text { No. } 4 \\
& \text { No. } 16 \\
& \text { No. } 50 \\
& \text { No. } 100 \\
& \text { No. } 200
\end{aligned}
$$

\begin{tabular}{|c|c|}
\hline $\begin{array}{l}\text { GRADATION } \\
\text { No. } 1\end{array}$ & $\begin{array}{c}\text { DESIGNATION } \\
\text { No. } 2\end{array}$ \\
\hline $\begin{aligned} & 100 \\
90 & -100 \\
20 & -55 \\
0 & -15 \\
0 & -5 \\
& -\end{aligned}$ & $\begin{aligned} &- \\
&- \\
& 100 \\
& 85-100 \\
& 10-30 \\
& 0-10\end{aligned}$ \\
\hline
\end{tabular}

$$
\begin{aligned}
& 100 \\
95 & -100 \\
55 & -75 \\
12 & -30 \\
3 & -10 \\
0 & -3
\end{aligned}
$$

AGGREGATE FOR DBST:

PERGBNT PASSING D MESH SIEVES

SIEVE DESIGNATION 
A P P E N D I X II

MATERIALS 


\section{APPENDIX I}

MONTHLY PERSONNEL COUNT

U.S. CORPS OF ENGINEERS:

Third

Country

Date Americans Nationals Afghans
AHC - HIQ:

Third

Country

Americans Nationals Afghans

1966

\begin{tabular}{|c|c|c|c|c|c|c|}
\hline August & - & 1 & - & 4 & 2 & 44 \\
\hline September & 4 & 6 & 19 & 11 & 11 & 607 \\
\hline October & 4 & 6 & 20 & 14 & 12 & 1,016 \\
\hline November & 5 & 6 & 22 & 15 & 21 & 1,157 \\
\hline December & 4 & 6 & 22 & 15 & 20 & 765 \\
\hline 1967 & & & & $\cdot$ & & \\
\hline January & 4 & 6 & 22 & 16 & 23 & 660 \\
\hline February & 5 & 5 & 22 & 15 & 23 & 619 \\
\hline March & 5 & 5 & 22 & 17 & 23 & 1,039 \\
\hline April & 5 & 5 & 23 & 17 & 23 & 1,231 \\
\hline May & 5 & 5 & 24 & 17 & 23 & 1,246 \\
\hline June & 5 & 5 & 24 & 19 & 23 & 1,342 \\
\hline July & 5 & 4 & 24 & 21 & 24 & 1,446 \\
\hline August & 5 & 4 & 24 & 22 & 24 & 1,284 \\
\hline September & 5 & 4 & 24 & 21 & 24 & 1,262 \\
\hline October & 5 & 4 & 24 & 16 & 22 & 239 \\
\hline November & 4 & 4 & 24 & 10 & 17 & 129 \\
\hline
\end{tabular}




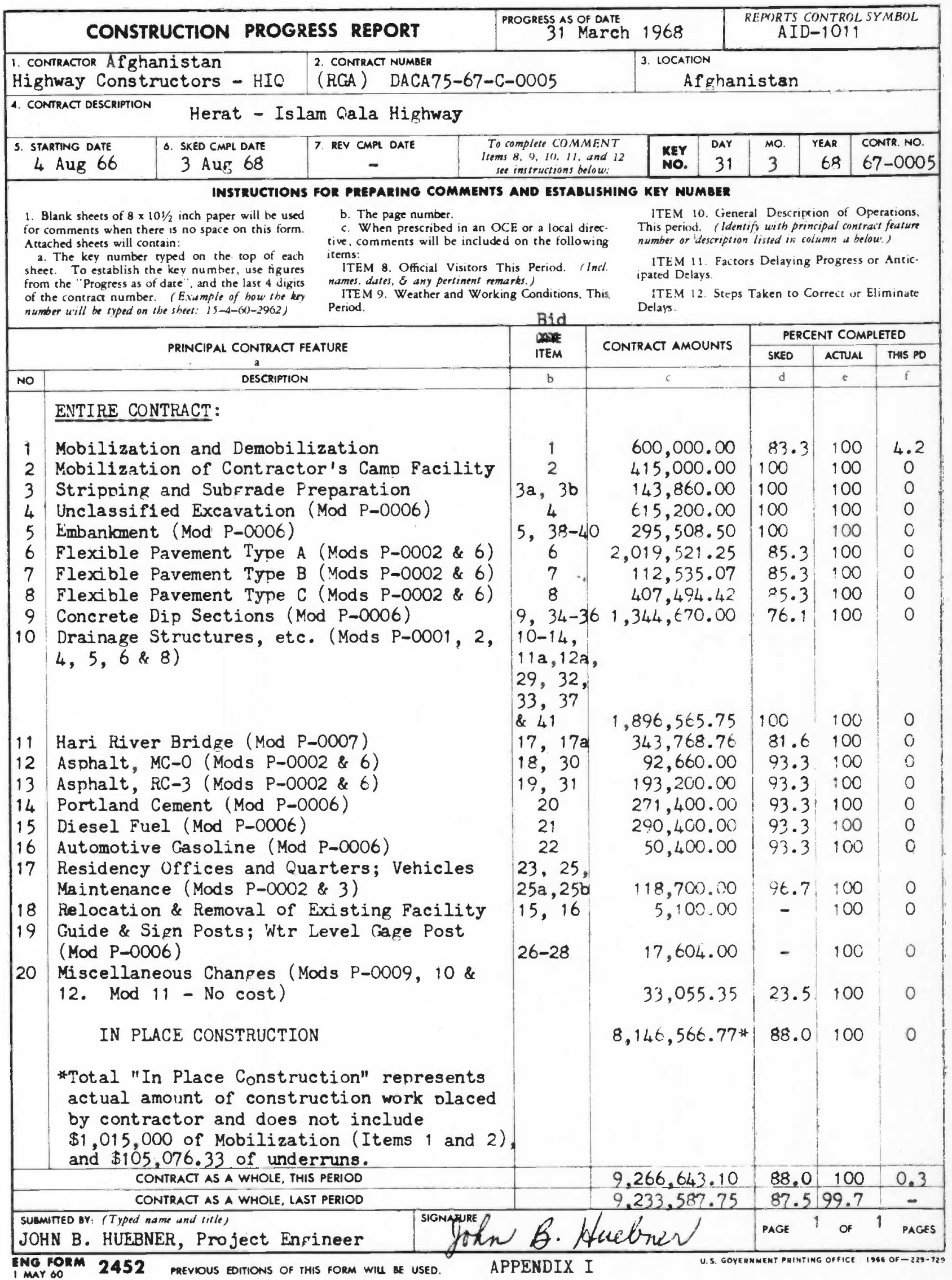




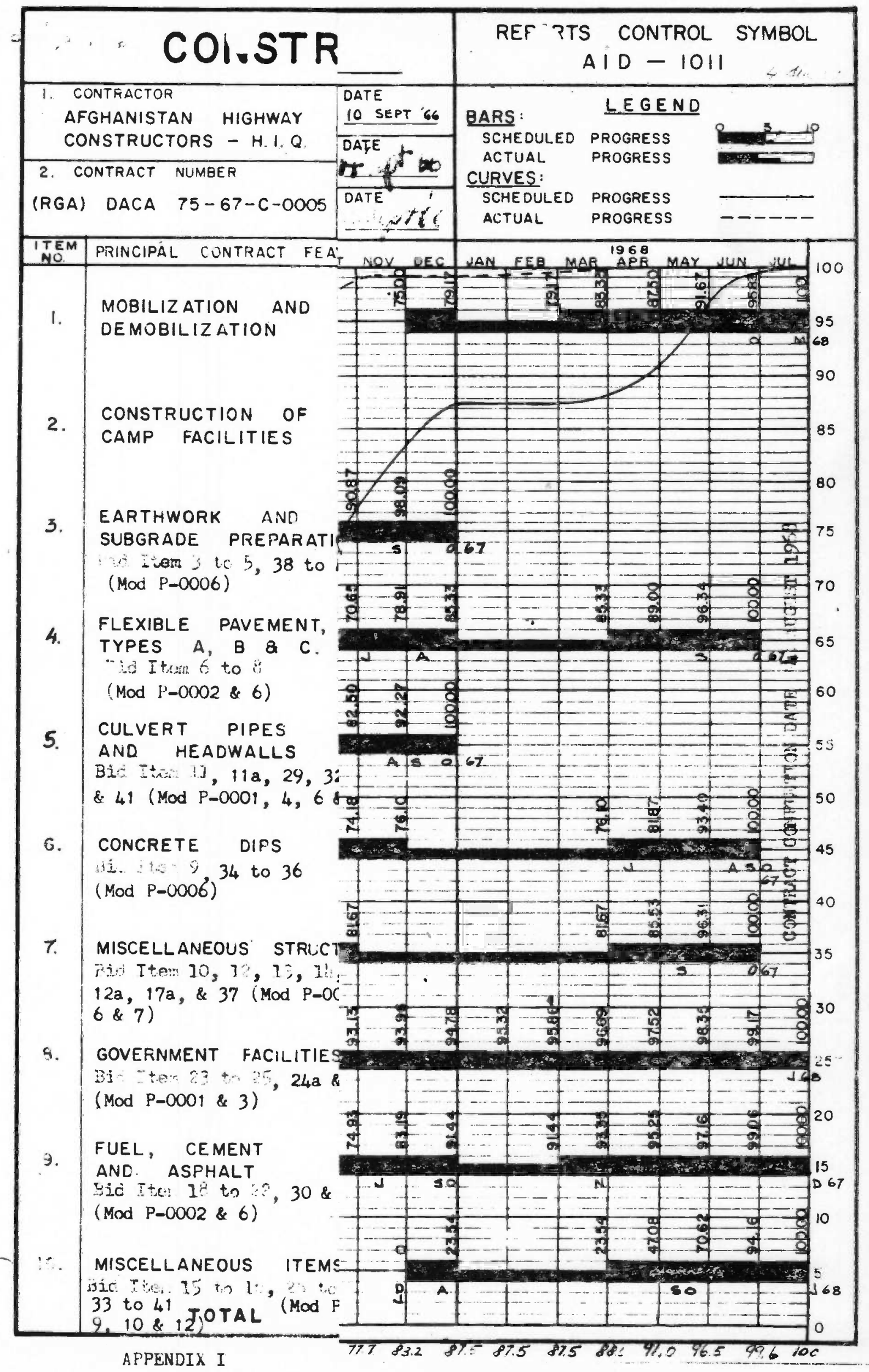


A P P ENDIX VII

PREVENTIVE MA INTENANCE AND

IMPROVEMENT PROGRAM 
APPENDIX VII

\section{PREVENTIVE MAINTENANCE AND IMPROVEMENT PROGRAM}

\section{FOR HERAT-ISLAM QAIA ROAD}

\section{INTRODUCTION}

a. The highway from Herat to Islam Qala is now a part of Afghanistan. As it now is, it provides a high speed road for people and goods to the Iranian border. But, no construction lasts forever; the life of this road will be entirely determined by the quality and quantity of maintenance given to it. Without a good maintenance program, this road cannot be expected to give good service for two years. There is too large an investment of time and money represented here for it to be allowed to deteriorate. Much of the required maintenance is the same as that required for any asphalt road and can be found in standard textbooks and manuals. But, because of the particular drainage problems and soil conditions to be found on this road, there are also many things that apply to this road alone. This manual is specifically designed to point up the areas where this road requires special attention.

b. The road maintenance cannot be done by one man, no matter how well trained, if he is not supported by a skilled and well equipped organization. He must have adequate equipment, kept in good condition with a supply of spare parts and supplied with adequate supplies to keep it running and on the road. Equipment deadlined for lack of fuel, or spare parts, or through being damaged by an inexperienced operator, is of no use to anyone. The organization; equipment and supplies detailed in this booklet are considered to be the absolute minimum that will insure the usability of the road; if they are not provided or not supported by adequate funds, this road will not last.

\section{PREVENTIVE MAINTENANCE, GENERAL}

a. The supervisor in charge of maintenance must thoroughly familiarize himself with the specifications and as-built drawings for this project. A large scale map, wall mounted, should be prepared showing the location and number and size of pipe of each culvert (Appendix A), and the length and design of each dip section (DBST, concrete without shoulder protection and concrete with shoulder protection), interceptor ditches, diversion dikes, 
channel changes, " $J$ " sections and bridges (Figure l, map).

Critical drainage areas where the road is most susceptible to damage by heavy runoff during the rainy seasons should be indicated.

b. The Maintenance Supervisor must thoroughly review and familiarize himself with the field conditions of drainage and construction of the road. Existing drainage conditions before channels intercept the newly constructed dikes, channels and interceptor ditches should be observed and studied since maintenance will be required to prevent changes in the existing flow and drainage pattern during the rainy season.

\section{MAINTENANCE OF CHANNELS, DIVERSION DIKES AND INTERCEPTOR DITCHES}

These structures are designed to insure that water crosses the road where intended. They must be kept in at least as good a condition as when built or they may malfunction. The maintenance required is removing deposits of silt from the channels and replacing eroded sections of dikes. The system will be improved if all the soil removed from the ditches is used to heighten the mounds and dikes. Most of this work can be done by unskilled labor, using nothing but shovels and hand tampers. This is the most important aspect of maintenance for this road and will require close supervision to insure that the available labor is effectively utilized.

a. Continual maintenance will be required during and after the rainy season, when sections of the dikes will be eroded and weakened, ditches and channels filled up by deposits of silt and gravel. Maintenance will also be required during the dry season since the prevailing winds and soil conditions tend to deposit large amounts of silt in the channels and interceptor ditches and unless removed will change the existing designed drainage pattern.

b. Channel and dikes are designed and constructed to intercept the existing wadis and drainage ditches. Critical points, where the channels intercept the existing drainage flow or where a change in direction of $f$ low has been initiated to carry the runoff to the culverts, will require frequent inspection to see that the excavation of channels and repair of dikes are adequate to prevent any change in the designed runoff pattern. If the existing wadis, designed channels and dikes are allowed to fill up or erode, the runoff will not go to the dip sections and culverts and severe damage to the traveled way will occur. This would necessitate extensive and costly repairs. 
c. In all probability the present existing wadis, designed channels and dikes will require repetitious repair and maintenance. Much of this repetition can be eliminated if the maintenance engineer will place either grouted or loose riprap protection in the areas which are extensively eroded or damaged during the runoff period.

d. Tractor drawn scrapers, dozers and graders may be used for the maintenance of the channel changes, diversion dikes and interceptor ditches. The rainy season is of a short duration and is followed by a 10 -month ideal working period. The drainage system should be in perfect condition before any rains start.

e. If the channels, dikes and interceptor ditches are not properly maintained, extensive damages to the roadway will occur and costly repairs to the subgrade, subbase, base and paved surface will be required to restore the road to its original status.

\section{MA INTENANCE OF CULVERTS}

a. Culvert installations for this road have been designed and constructed to require a minimum amount of maintenance and to be as trouble free as possible. Flow design and sizes of culverts were basically computed by visual observation in the field during the past rainy season when the flow design was increased approximately 200 percent over the original drainage design. Massive masonry headwalls with cutoff walls extending $60 \mathrm{CM}$ below invert of pipe along with grouted wing walls and aprons constitute a strong and durable structure.

b. The first and most important requirement for the maintenance of a drainage structure is to keep it clean. Heavily silted or partially filled up culverts may cause damages not only to the road and culvert at the principal location but may also cause damage to the roadway in other areas. With the capacity of the culvert thus decreased, heavy silting and deposits of gravel and sand will occur in the interceptor ditch; this may, in turn, cause the water to overflow the road, even at a considerable distance from the culvert location. Any water overflowing the road will cause extensive damage. If one culvert becomes blocked, the water may flow along the road to other culverts which will then be overloaded. In this way the road will be damaged also in other places. Culverts will have to be periodically cleaned during the entire year, not only during and after the rainy seasons. Silting and blocking of the culverts occur during the dry season by the prevailing winds causing the fine silt to drift into the entrances 
of the culverts. The smaller diameter culverts can be effectively cleaned by using a long-handled hoe or shovel, or by pulling a scraper through with a rope; two men are required for this job. Larger culverts are easily cleaned by one man with a shovel.

c. Erosion that occurs on the upstream side of the culverts may cause damage to the aprons, wing walls and riprap if not promptly repaired. Downstream scouring and turbulence will in all probability occur; this will cause extensive damage to the downstream aprons and wing walls through undercutting. As soon as erosion is detected, the hole should be filled with large rocks, damage to structures repaired and additional riprap installed along the end of the apron. Lack of adequate maintenance of this nature will start extensive erosion and undercutting of the headwall which, with the metal pipe, may collapse. Inlet and outlet ditches for the culvert will have to be daylighted or kept free flowing since any obstructions on the downstream side of the culvert will cause silting within the structure itself. Mortar joints for the headwalls should be repaired if cracked or loose. Timely and periodic minor repairs to the culverts must be made to insure that the road will be protected. Cleaning or repair of the culverts will be accomplished mainly by laborers except for daylighting which can be maintained by graders and scrapers.

d. In several instances, small dams have been built across the interceptor ditch just downstream from a culvert. These are to act as a sort of a safety valve and should fail before road damage occurs. They are primarily to divert the flow of water from smaller storms into the intermediate culverts. When a longer storm occurs, one that would overload a specific culvert, the dam will overflow and wash out before the culvert overloads. The excess water can then run downhill to another culvert which may not be overloaded. In case all culverts in a particular stretch become overloaded, at least the water will be concentrated at one point and only overflow the road in a small area. It is important that these dams be replaced when washed out, or subsequent smaller rains may concentrate enough water at low areas to cut the road. At the same time, the top of each dam must be below the height of the top of the accompanying culvert pipe or it will no longer serve as a safety valve.

\section{MAINTENANCE OF BRIDGES}

The reinforced concrete bridge is another strong and durable structure that was designed to give years of trouble-free service with a minimum amount of maintenance and no major repairs to the 
principal structure itself is anticipated. There are approximately four periodic maintenance services which will be required:

a. The backfilled embankments for the approaches to the bridge that will be affected by the flow of water in the Hari River are protected by large pieces of loose riprap; this should adequately protect the embankment from erosion. Little or no trouble is anticipated for the embankment at the east side of the river. Considerable attention should be given to the embankment on the west side of the river since this embankment is subjected to much more turbulence and a faster flow of water. Visual observation shows that there is considerable erosion on the upstream and downstream sides of this abutment. The Hari River is almost dry with only a small stream of water flowing in the river bed between the first of July and the first of December. During this period the Maintenance Supervisor should check the erosion that was incurred during the high water stage and make any necessary repairs. Since there is evidence of considerable erosion on the upstream and downstream sides of the protected abutment, a simple road improvement project would be to place loose riprap on the river bank for a distance of approximately 100 meters upstream and downstream during the dry period.

b. All expansion and control joints should be inspected periodically to determine if the asphalt sealer is cracked or sections of the joints unsealed or open. The joint should be thoroughly cleaned out and resealed with an approved joint sealer.

c. The metal railings of the bridge have been protected from erosion and rusting by painting first with red lead protective paint and a second coat of aluminum paint. This protective painting should give adequate protection for a two-year period. If scaling of the paint or rust signs appear on the railings, the areas involved should be thoroughly cleaned, removing all rust and scaling particles, and repainted to the original specifications.

d. Weep holes and drain holes installed in the concrete deck of the bridge should be kept clean so no water will stand on the surface of the bridge. Drainage gutters installed for the protection of the riprap at each approach to the bridge should be dept clean so water will flow as designed and not damage riprap protection. 


\section{A $\underline{G} \underline{\mathbf{E}} \underline{\mathrm{E}} \underline{\mathrm{M}} \underline{\mathrm{N}} \underline{\mathrm{T}}$ \\ BETWEEN}

THE ROYAI GOVERNMENT OF AFGHANISTAN

AND

TEE CORPS OF ENGINEERS, DEPARTMENT OF THE ARMY

OF THE UNTTED STATES OF AMERICA

RELATING TO THE CONSTRUCTION OF A ROAD IN WESTERN AFGHANISTAN

TISS AGLZE:LONI encered into between the Royal Government of Afghanistan, herein ramad Trae Government, and the CORPS OF ENGINEERS, Department of Arcy, of tize United States of America, herein named the Corpo, this Znembete day of 1966.

Uridas the terms of the Loan Agreement between the Royal Government of S.fir.nisican and the Untted States of Amerlca, acting through the Agency Iu: International Development (AID), the Royal Government of Afghanistan wili finance by means of Agency for Internatlonal Development Losn No. 306-H-006 the dollar costs of constructing a road from the C1ty of Herat in western Afghanlstan to the border of Iran which 18 to the West of the $\therefore$ iran town of Islam Qala, a distance of approximately 124 kilometers, (isercafter referred to as the B.I.Q. Road).

Pursuant to the terms of the above-mentloned Loan Agreement whlch has seen execuled between the Royal Government of Afghanistan and the Uniced Siaies, asiing through AID, the Government and the Corps hereby agree ait the Corps will, In behalf of and for the Government provide complete instruction management and engineering services in connection with the construction of the H.I.Q. Road. The scope of the work authorized for construction shall be encompassed by the contract documents prepared by the Corps in the design phase of the project and approved by the Royal Government of Afghanistan and AID.

Tris agreement is entered Into by the Corps at the request of AID pursunet to Section 607 of the Forelga Assistance Act of $1963 \mathrm{as}$ amended.

Ti.e Chlef of Englneers has designated the DIvision Englneer, U.S. Army Englneer Division, Mediterranean, Leghorn, Italy, or his authorlzed representative, to act for the Corps in discharging Its responsibility hereunder. It is understood that whenever the term "Division EngIneer" is used In this Agreement, it also means his authorized representative.

The Government hereby authorlzes the Minlster of Public Works to act for the Goverment in diecharging ite reoponelbilitied under this Agreement. 
The minister will designate a Llaison Officer who will be the point of contact between the Government and all representatives of the Corps.

Work undertaken in accordance with this Agreement shall be executed in accordance with the procedures and conditions outlined below.

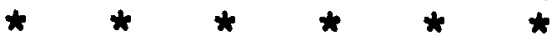

\section{ARTICLE I}

The services to be provided by the Corps shall conform to the terms and provisions of the H.I.Q. Loan Agreement. Such services shall consist of complete construction management and engineering services required for the complete construction of the H.I.Q. Road to include the following:

1. Supervise and control the engineering and construction portions of the project from completion of design through completion of construc$\because$ on. The Corps will insure the work is accomplished in accordance with cia best engineering standards properly applicable to such work.

2. Prepare and submit to the Government for approval of all tine proposed contractual documents, including the 11 st of proposers as compiled after formal pre-qualification and the formal Request for Proposals.

3. Request, receive, and evaluate proposals; negotlate and sign a contract after AID approval of the negotiated contract.

4. Accomplish the functions of a contracting officer for the Governient. The Contractor shall be given instructions only by the Contracting officer. The functions to be performed by the Contracting Officer include but are not limited to the following:

a. Supervision of the Contractor's work to insure compliance with contract drawings and specifications.

b. Approval of the contractor's construction schedule.

c. Issuance of change orders as specified in Article II.

d. Issue decisions in contractual disputes.

e. Certification of progress payments to be paid to the contractor.

f. At the Government's request assist in arrangements to procure maintenance equipment under an approved maintenance program for the road.

8. Appoint the Government's representative to an arbitration panel if one is required pursuant to the dleputes clause of the conetruction contract General Provisions.

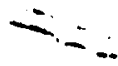


h. Socve as inftial beneficlary of a letter of credit to bo established pursuant to the special barter transaction. The Contracting Officer will assign this Letter of Credit to the construction contractor when contract award 18 made.

5. Submit to the Government the plan of the proposed sequence of work after the Contracting Officer's approval of the contractor's proposcd conit. uction prograse shedula.

6. Submi- to the Government quarterly reports of the contractor's progress and otizer reports required under the terms of the Loan Agreement and implementing letters.

7. At the time of completion of the construction contract, furnish to the Government reproducible as-bullt drawings, and any other pertinent documents, with the transfer of the completed construction.

8. Upon final completion of the work or any portion thereof as sat forti ir tia construction contract documents, inspect the completed work jointly with the Government and USAID and accept such work as in its judrment complies with the terms of the construction contract.

9. Upon acceptance of the completed work or -any portion thereof, tise Governmest will have access to all, or that portion, of the road and will assume responsiblitity for the maintenance thereof.

\section{ARTICLE II}

1. The Contracting Officer will have sole responsibility to issue changes within the contract scope based upon engineering or construction considerations. He may al so negotiate and execute contract modifications for such changes, coples of which will be submitted to the Royal Government OE Afghanistan and AID. The Contracting Officer w11l have no authority to otherwise change the plans and specifications. In no event will the Contracting Officer make any changes which will cause funds for contract and contingencies to be exceeded.

2. Any changes requested by the Government or deemed necessary by the Contracting officer that are outside the contract scope or which regitise funds beyond that amount of dollars and local currency called for by the contract, must be jointly approved by the Government, the Contracting OEficer and AID. Any additional funds 00 required must be made avallable by the Government prior to formal contract action. 


\section{ARTICLE III}

1. The dollar costs of the construction contract shall be paid in the following manner:

a. Upon approval of the proposed construction contract, the Government will request AID to issue a Letter of Commitment to a United States bank for the full dollar amount necessary for the contract; plus contingencies, but less the amount to be otherwise made available pursuant to Paragraph 3 below.

b. Upon notification that the Letter of Commitment has been issued, the Government will open an irrevocable Letter of Credit in the same United Stati's bank in favor of the approved contractor for an amount equivalent to that furnished through the above-mentioned Letter of Commitment.

c. The contractor shall periodically submit copies of invoices and other documents for dollar payments to the Contracting Officer. Upon certification by the Contracting Officer on each copy that these charges are legitimate and parable, the invoices may be processed for payment.

2. The afghani cost of the construction contract shall be paid in the following manner:

a. The amount of local currency required for construction to: one year, as detcrmined on the basis of Afghan fiscal years, shall bc deposited by the Covernment in the Da Afghanistan Bank in an account established in the name of the Finance and Accounting Officer, U.S. Army Engineer Division, Mediterranean. For the first year of construction, to this amount will be added sufficient local currency to allow the contractor to de-mobilize in the event afghani for the next year's construction costs are not forthcoming from the Government for any reason whatsoever.

b. The Contracting Officer will determine the amounts the Government should deposit the first year and each year thereafter and shall advise the Government of these amounts at least six months prior to the date the deposit is to be made. Deposit should be made by I May of $: \mathrm{ich}$ construction year, except for first year when deposit must be made info ie construction contract is awarded.

c. The approved contractor shall periodically submit copies of invoices for afghani costs to the Contracting Officer who shall certify on each copy that these charges are legitimate and payable before processing them for payment. 
3. Certain commodities consisting of asphalt, Portland cement, and petroleum products to be used in the construction of the project wil not be paid for from funds to be supplied through either of the above arrangements. By agreement with AID, these comodities will be pald for under a separate Letter of Cred1t established pursuant to a specilal barter transaction.

4. Dollar coete Incurred by the Corpe for personal enrvices in the administration and supervision of the profect required herein will be pald by AID and shall not be charged to the funding referred to hereln.

$$
\text { * } \quad * \quad * \quad * \quad * \quad *
$$

This agreement may be modified or amended by written agreement between the Government and the Corps, subject to prlor written approval by AID, and may be 80 modified in the event additional objectives are mutually agreed to and adopted so long as the scope of services approved by AID and the funds provided by AID are not exceeded. In the event circumstances are such that either the Government or the Corps deems it necessary or desirable to cerminate this agreement befoce completion of the servicea provided herein, the Govermment and the Corps will.consult in advance so that the parties may make personnel and other adjustments in their operations in light of auch termination.

IN WITNESS WHEREOR the parties have algned this Agreement on the date and year flrst above written.

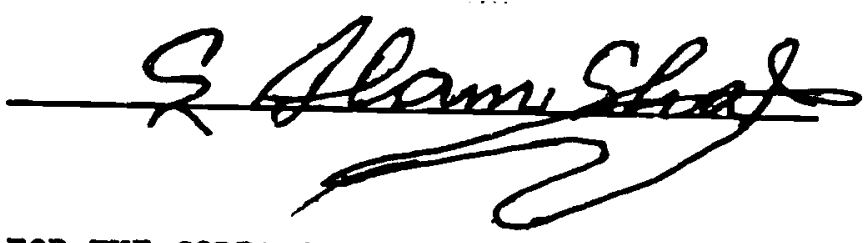

POR THE CORPS OF ENGINEERS, DEPARTMENT OF THE ARMY, UNITED STATES OF AMERICA

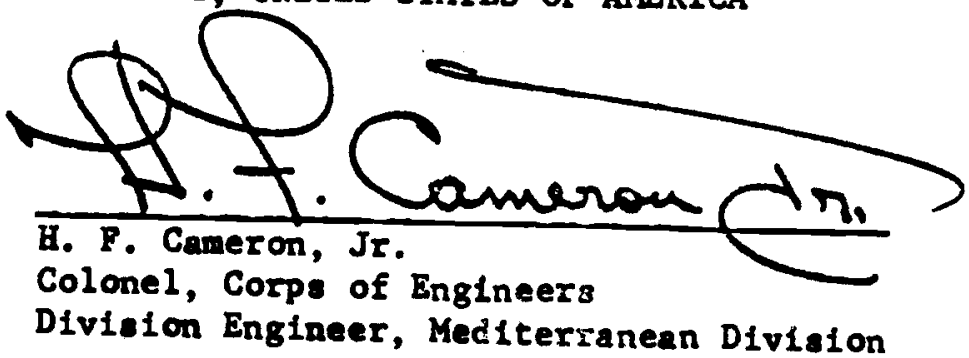


A P P E N D I X VI

LOAN AGREEMENT BETWEEN RGA AND USA

WORK AGREEMENT BETWEEN RGA AND CORPS 
A. I.D. Loan No, 306-H- -006

\title{
LOAN AGREEMEN T
}

\author{
Afghanistan - Herat-Islam Qala Road
}

BETWEEN THE

ROYAL GOVERNMENT OF AFGHANISTAN

AND THE

UNITED STATES OF AMER ICA

Dated: January 12, 1965 
TABLE OF CONTENTS

Section Number

Title

Page

ARTICLE I. THE LOAN

SECTION 1.1.

The Loan

SECTION 1.2.

The Project

1

1

ART ICLE II TERMS OF REPAYMENT AND INTEREST

$\begin{array}{ll}\text { SECIION } & 2.1 . \\ \text { SECIION } & 2.2 . \\ \text { SECTION } & 2.3 . \\ \text { SECTION } & 2.4 .\end{array}$

Interest

Principal

Application and Place of Payment

Prepayment

2

2

3

ARTICLE III CONDITIONS PRECEDENT

SECTION 3.1.

SECTION 3.2 .

SECTION 3.3.
General Conditions Precedent to Financing Additional Conditions Procedent to Financing Specific Items Terminal Dates for Fulfillment of Conditions Precedent

4

4

5

ARTICLE IV DISBURSEMENTS

SECTION 4.1.

SECIION 4.2.

Request for Letters of Commitment

Other Forms of Di sbursement

Terminal Date for Requests for Letters

of Commitment and for Disbursements

ART ICLE V MISCELLANEOUS

SECTION 5.1 . SECTION 5.2. SECIION 5.3. SECTION 5.4. SECTION 5.5 .
Eligibility Date

Ocean Shipments

Use of Representatives

Communications

Standard Provisions Annex 
AGREEMENT made and entered into as of the day of July, 1964, between the Royal Government of Afghanistan ("Borrower") and the UNITED STATES OF AMERICA, acting through the Agency for International Development ("A. I.D.").

\section{ARTICLE I. The Loan.}

SECTION 1.1. The Loan. Subject to the terms and conditions of this Agreement, A.I.D. agrees to lend to Borrower pursuant to the Foreign Assistance Act of 1961, as amended, and Borrower agrees to borrow from A.I.D. up to Seven Million Seven Hundred Thousand United States dollars $(\$ 7,700,000)$ for the foreign exchange costs of goods and services required for the Project as defined in Section 1.2. (the "Loan"). The funds disbursed hereunder are herein referred to as "Principal". Goods and services financed hereunder are herein referred to as "Eligible Items". References herein to the "Borrower Country" are to Afghanistan.

SECTION 1.2. The Project. As used in this Agreement, "Project" shall mean: The construction of an asphalt surfaced road 5.52 meters wide, with 1.2 meter shoulders on each side, from the city of Herat in western Afghanistan to the border of Iran just west of the Afghan town of Islam Qala, a distance of approximately 124 kilometers, and road maintenance equipment in an amount not to exceed $\$ 250,000$. 
ART ICLE II. Terms of Repayment and Interest

SECTION 2.1. Interest. Borrower shall pay interest semiannually to A.I.D. in United States dollars on the unrepaid Principal, and on any interest due and unpaid, computed on the basis of a 365 day year and at the rates hereinafter stated. The first such payment shall be due and payable six (6) months after the first disbursement hereunder. Interest shall accrue from the dates of the respective disbursements at the rate, for the ten (10) years following the date of such first disbursement, of three fourths of one percent ( $3 / 4$ of $1 \%)$ per annum and thereafter at the rate of two percent (2\%) per annum. Disbursements shall be deemed to occur on the dates on which payments by A.I.D, are made to Borrower or to its designee or to a banking institution pursuant to a letter of "commitment.

SECTION 2.2. Principal. Borrower shall repay the Principal to A.I.D. in United States dollars in semi-annual installments in accordance with the terms of this Agreement and the amortization schedule to be provided the Borrower by A.I.D. after all disbursements have been made. Such amortization schedule shall provide for sixty-one (61) approximately equal semi-annual payments of Principal and interest, the first installment to be payable ten (10) years after the date of the first disbursement hereunder.

SECTION 2.3. Application and Place of Payment. All payments shall be applied first to accrued interest and then to Principal. Except as A.I.D. may otherwise direct in writing, payments shall be made to the Controller, USAID Mission, Kabul, Afghanistan, and shall be deemed to have been made when received by A.I.D. at such address. 


\section{$-3-$}

SECTION 2.4. Prepayment. Borrower shall have the right to repay all or any part of the Principal, without penalty, on any date on which interest is due. Any prepayment shall be applied to the installments of Principal and interest provided for in the schedule to be furnished by A.I.D. pursuant to Section 2.2. in inverse order of their maturity which installments shall further be duly adjusted to take account of the resulting reduction in Borrower's interest obligation hereunder. 
ARTICLE III. Conditions Precedent.

SECTION 3.1. General Conditions Precedent to Financing.

Prior to the issuance of any letters of commitment and to any disbursement hereunder, Borrower shall furnish A.I.D. in form and substance satisfactory to A.I.D.:

(a) An opinion of the senior legal officer of the Borrower or of other counsel satisfactory to A.I.D. that this Agreement has been duly authorized or ratified by and executed on behalf of Borrower and constitutes a valid and legally binding obligation of Borrower in accordance with its terms;

(b) Evidence of the authority of the person or persons who will act for Borrower pursuant to Section 5.4 and a specimen signature of each such person certified as to its authenticity by either the person who renders the said legal opinion or the person who executes this Agreement;

(c) Evidence of the availability of funds, other than those to be provided hereunder, necessary for the construction of the Project;

SECTION 3.2. Additional Conditions Precedent to Financing Specific Items. Upon completion of a plan and time schedule for the construction of the road the Borrower may obtain letters of commitment for each Eligible Item as follows: 


$$
-5-
$$

(a) Construction of the road.

(i) Evidence of arrangements satisfactory to A.I.D. for the construction of the road. Such arrangements shall provide that, to the maximum extent practicable, the Principal will not be used to finance the procurement of goods or services otherwise eligible for financing hereunder, if comparable goods or services are produced and available in the Borrower Country in sufficient quantity, of satisfactory qualify and at a reasonable price;

(ii) Evidence that all privileges, easements, including rights of way, and permits necessary for the construction of the road and the subsequent operation thereof have been obtained;

(iii) Evidence that the Borrower has put into effect a program satisfactory to A.I.D. for the maintenance of U.S. assisted highways;

(iv) Evidence satisfactory to A.I.D. that there shall be a common meeting point of the roads of the Borrower and the Government of Iran at their border in the vicinity of Islam Qala.

(b) Maintenance Equipment.

Evidence that arrangements satisfactory to A.I.D. have been made to procure the maintenance equipment.

(c) Such additional plans, specifications or other documents for the Project as A.I.D. may requcst. 
SECTION 3.3. Terminal Dates for Fulfillment of Conditions Precedent.

(a) Except as A.I.D. may otherwise agree in writing, if the conditions established by Sections 3.1. and 3.2. (a) have not been satisfied by June 1, 1965, A.I.D. at anytime thereafter may at its option terminate this agreement by written notice to Borrower. Upon receipt of such notice all obligations of Borrower and A.I.D. under this agreement shall cease.

(b) Except as A.I.D. may otherwise agree in writing, if the conditions established by Section 3.2. (b) have not been satisfied by August 31, 1967, A.I.D. at anytime thereafter may at its option terminate its obligation to finance the procurement of road maintenance equipment under this agreement by written notice to Borrower. 
ARTICLE IV. Disbursements:

SECTION 4.1. Request for Letters of Commitment. To obtain disbursements, Borrower may from time to time request A.I.D. to issue letters of commitment to one or more banking institutions in the United States designated by Borrover and satisfactory to A.I.D., committing A.I.D. to reimburse such bank or banks for payments made, through letters of credit or otherwise, to Borrower or any designee of Borrower, pursuant to such documentation requirements as A.I.D. may prescribe. Banking charges incurred in connection with letters of commitment and disbursements shall be for the account of Borrower and may be financed hereunder. SECTION 4.2. Other Forms of Disbursement. Disbursements may also be made by such other means as Borrower and A.I.D. may agree to in writing.

SECTION 4.3. Terminal Date for Requests for Letters of Commitment and for Disbursements. Except as A.I.D. may otherwise agree in writing, no letters of cormitment shall be issued in response to requests received by A.I.D. after July 1,1967 and no disbursements shall be made against documentation received by A.I.D. after December 31, 1967. 
ARTICIE V. Miscellaneous

SECTION 5.1. Eligibility Date. As used in this Agreement, "Eligibility Date" shall be the date of this Agreement.

SECTION 5.2. Ocean Shipment. Borrower shall ensure that at least fifty percent, (50\%) of the gross tonnage of all goods (computed separately for dry bulk carriers, dry cargo liners and tankers) financed hereunder which shall be transported on ocean vessels shall be transported on privately owned United States flag commercial vessels. No goods may be financed hereunder which are transported on any ocean vessel (a) which A.I.D., by written notice to Borrower, has designated as ineligible to carry A.I.D.-financed goods or (b) which has been chartered for the carriage of A.I.D.-financed goods unless such charter has been approved by A.I.D.

SECTION 5.3. Use of Representatives. (a) All actions required or permitted to be performed or taken under this Agreement by Borrower or A.I.D. may be performed by their respective duly authorized representatives.

(b) Borrower hereby designates as its representative with authority to designate in writing other representatives of Borrower in its dealings with A.I.D. Borrower's representatives designated pursuant to the preceding sentence, unless A.I.D. shall be otherwise notified in writing, shall have authority to agree on behalf of Borrower to any 
modification of this Agreement which does not substantially increase Borrower's obligations hereunder. Until receipt by A.I.D. of witten notice of revocation by Borrower of the authority of any of its representatives, A.I.D. may accept the signature of any such representative on any instrument as conclusive evidence that any action effected by such instrument is authorized by Borrower.

SECIION 5.4. Communications. Any notice, request or other communication given, made or sent by Borrower of A.I.D. pursuant to this Agreement shall be in writing and shall be deemed to have been duly given and delivered to the party to which it is addressed when delivered by hand, mail, telegram, cable or radiogram to such party at the following address:

To Borrower:

Mail Address:

Loan Division Treasury Department Ministry of Finance Kabul, Afghanistan

Cable Address:

Ministry of Finance

To A.I.D. (two copies):

Kabul, Afghanistan

Mail Address:

Agency for International Development

Department of State

Wa shington, D.C. 20523

U.S.A.

Cable Address:

AID

Washington, D.C., U.S.A.

Borrower, in addition, shall provide the U.S. A.I.D. Mission, Kabul, Afghanistan with a copy of all communications or documents sent to A.I.D. Other addresses may be substituted for the foregoing upon written notice. 
All communications and documents submitted to A.I.D. hereunder shall be in English and all technical and engineering specifications therein shall be in terms of United States standards, except as A.I.D. may otherwise agree in writing.

SECTION 5.5. Standard Provisions Annex. A Standard Provisions Annex is attached to this Agreement and, is hereby made a part hereof. Terms used therein shall have the same meaning as such terms are given in this Agreement. References therein to "the Agreement" are to this Agreement.

ROYAL GOVERNMENT OF AFGHANISTAN

By: Sayid Kassen Rishtiya (Name)

Title: Minister of Finance

UNITED STATES OF AMER ICA

BY: John M. Steeves (Name) 


\begin{tabular}{|c|c|c|c|}
\hline A10.2-4 (Lea) & $\begin{array}{l}\text { PARTICIP - ING AGEMCY SERVICE AGREEMENT } \\
\text { WITH }\end{array}$ & $\begin{array}{l}\text { SA CONTROL NO. } \\
\text { NESA (DB) - I2-OO } \\
\text { Amend. } \# 3\end{array}$ & $\begin{array}{l}\text { Page } 1 \\
\text { of } 5 \text { Pages }\end{array}$ \\
\hline $\begin{array}{l}\text { SPECIAL } \\
\text { PROVISLONS }\end{array}$ & $\begin{array}{c}\text { DEPARTMENT OF TTEE ARMY } \\
\text { SPECIAL PROVISIONS }\end{array}$ & Afghanistan & $34-2-60009$ \\
\hline
\end{tabular}

\author{
A G $\underline{R} \underline{\mathrm{E}} \underline{\mathrm{E}} \underline{\mathrm{M}} \mathrm{E} \underline{\mathrm{N}} \underline{\mathrm{T}}$ \\ BETWEIN \\ THE ROYAL GOVERTMENT OF AFGHANISTAN \\ AND \\ THE CORPS OF ENGINEERR, DEPARTMENT OF THE ARMY \\ OF THE UNITIED STATES OF AMERICA
}

RELATING TO THE CONSTRUCTION OF A ROAD IN WESTERN AFGHANISTAN

THIS AGREEMENT entered into between the Royal Government of Afghanistan, herein named The Government, and the CORPS OF ENGINEERS, Department of the Army, of the United States of America, herein named the Corps; this day of 1965.

\begin{abstract}
Under the terms of the Loan Agreement between the Royal Government of Afghanistan and the United States of America, acting through the Agency for International Development (AID), the Royal Government of Afghanistan will finance by means of Agency for International Development Loan No. 306-H-006 the dollar costs of constructing a road from the City of Herat in western Afghanistan to the border of Iran which is to the west of the Afghan town of Islam Qala, a distance of approximately 124 kilometers. (Hereafter referred to as the H.I.Q. Road).
\end{abstract}

Pursuant to the terms of the above-mentioned Loan Agreement which has been executed between the Royal Government of Afghanistan and the United States, acting through AID, the Government and the Corps hereby agree that the Corps will, in behalf of and for the Government provide complete construction manegement and engineering services in connection with the construction of the $F . L_{0}$. R.oec. The scope of the work authorized for construction shall be encompassed by the contract documents prepared by the Corps in the design phase of the project and approved by the Royal Government of Afghanistan and AID.

This Agreement is entered into by the Corps at the request of AID fursuant to Section 607 of the Foreign Assistance Act of 1963 as ainended.

The Chief of Engineers has designated the Division Engineer, J.S. Army Engineer Division, Mediterranean, Leghorn, Italy, or his authorized representative, to act for the Corps in discharging its responsibilities hereunder. It is understood that whenever the term "Division Engineer" is used in this Agreement, it also means his authorized representative. 
6. Submit to the Government quarterly reports of the contractor's progress and other reports required under the terms of the Ioan Agreement and implementing letters.

7. At the tim of completion of the construction contract, furnioh to the Government reproducible as-bullt drawings, and any other pertinent documents, with the transfer of the completed construction.

8. Upon final completion of the work or any portion thereof as set forth in the construction contract documents, inspect the completed work jointly with the Government and USAID and accept such work $a s$ in its judgement complies with the terms of the construction contract.

9. Upon acceptance of the completed work or any portion thereos, the Government will have access to all, or that portion, of the road and will assume responsibility for the maintenance thereof.

\section{ARTICLE II}

1. The Contracting officer will have sole responsibility to 1s sue changes within the contract scope based upon engineering or construction considerations. He may also negotiate and execute contract modifications for such changes, coples of which will be submitted to the Royal Government of Afghanistan and AID. The Contracting Officer will have no authority to otherwise change the plans and speciffcations. In no event wili the Contracting officer make any changes which will cause funds for contract and contingencies to be exceeded.

2. Any changes requested by the Government or deemed necessary by the Contracting officer that are outside the contract scope or which require funds beyond that amount of dollars and locai currency called for by the contract, must be jointly approved by the Government, the Contracting Officer and AID. Any additional funds so required must be made available by the Government prior to formal contract action.

\section{ARTICIE III}

1. The dollar costs of the construction contract shall be paid in the foliowing manner:

a. Upon approval of the proposed construction contract, the Government will request AID to issue a Letter of Commitment to a United States bank for the full doliar amount necessary for the contract, plus con tingencles, but less the amount to be otherwise made available pursuant to Paragraph 3 below.

b. Upon notification that the Letter of Commitment has been issued, the Government will open an irrevocable Ietter of Credit in the same United States Bank in favor of the approved contractor for an amount equivalent to that furnished through the above-mentioned Letter of Commitment. 
the Government and the Corps will consult in advance so that the parties may make personnel and other adjustments in their operations in light of such termination.

IN WITNESS WHEREOF the parties have signed this Agreement on the date and year first above written.

FOR THE ROYAL GOVERNMENT OF AFGHANISTAN

FOR THE CORPS OF ENGIIEERS, DEPARTMENT OF THE ARMY, UNITEL STATES OF AIERICA.

A. D. Chaffin, Jr.

Colonel, Corps of Engineers

Division Engineer, Mediterrarean Division 


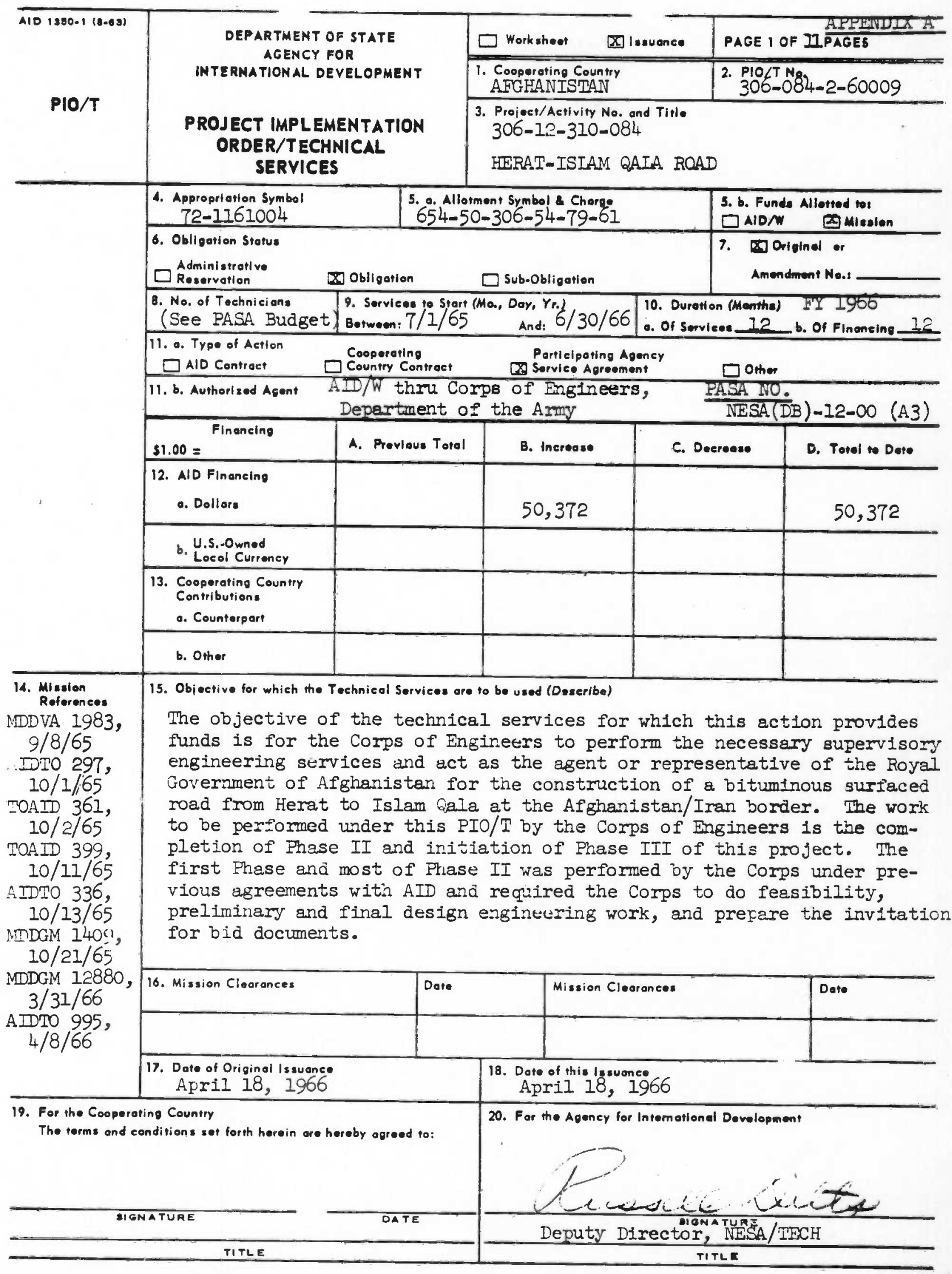




\section{PROJECT IMPLEHENTATION ORDER/TECHNICAL SERVICES}

\begin{tabular}{|c|c|}
\hline [ Worksheet & PAGE 3 OF "II PAGES \\
\hline $\begin{array}{l}\text { Cooperating Country } \\
\text { AFGHAiNISTAN }\end{array}$ & $\begin{array}{l}\text { 10/T Na. } \\
\text { 306-084-2-60009 }\end{array}$ \\
\hline \multicolumn{2}{|l|}{$\begin{array}{l}\text { Prolect/Aetivity No. and Tirle } \\
306-12-310-084\end{array}$} \\
\hline \multicolumn{2}{|c|}{ HWPAT-ISIAM QAIA ROAD } \\
\hline
\end{tabular}

22. Equipment and Supplies (Reloted to the wervices dascribed in Block 21 and to be procured outsids the Cooperating Country by the aupolier of these services)

\section{A. (1) Quantity (2) Deseription}

(3) Estimoted Costs

The Corps will be responsible for and authorized to procure such items/ to the performence of its worl and the support of its staff firom the furds providrd und. F this PIO/T. xeept as may be provided for und a f future constmation contract Any and ali propenty purchased under this IIO/T shall be titld under USAID/Af fhanistan and ahall be returned to TSAID/A on tise completion oi this assignm:nt. Any oth $r$ property or commoditiss brrught inte Afghanjstan by the COE for utjlization on ihis projict, and which wes not fjnenced lindar this agr sment, may br. removed from 8. Finoneing Coste of Equipment ond Supolie.

Afghanistan by the U,S Army Corps of Enginiors upor (1) 8y AID - \$82L. (Cr. atta Sudget) (2) By Cooporoting Country completion of the projuc

\section{Instructions to Authorizad Agarit}

AID/W will nezotiate and Execute a PASA amencment with the Corps of Enginsers for the sirvices request.d herein.

This action is as noted above, for the sompletikn of Fiese II and initiation of Phys: III of a thres-phese program of grant assistance to the Royai Government of Afghanistin, Employ ing tile Corps to jrovide the necessar engineering end technicil servi-es for the construction of the herat-Islam Qala roal. AID Loan No. 306-H-006 in the amount o: $37,700,000$ to RGA was signed on Jimary 12. 1965. to finsnce the dollar construction costs of the Herat-Islam Qala Foad project. It will be supplis-

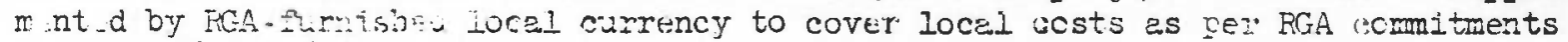
in the 306-H-006 Loun Agreenent.

24. Speciol Provisions

$\therefore$. This PIO/T aithorizes tine continuation in FY 1966 a $j$ services performed by the torps of Engtnects. I A. under FIO/T 306-084-2-50003, as amended, and its r. lated Participating Agricy Service Agrement and is suzject to the same tamas, conaitions and arranguments. Exept as modificd herein

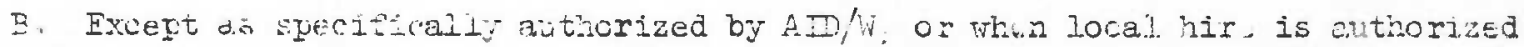
und I the terms of a contract with a U.E. supplier services authorized undrr this $\mathrm{PIC/T}$ must be obtidned from IT.S sources.

C. Except as specifinail; euthowized by AID/W the purchase of commoditis putioniz under this FIO/T will be limited to the United Statis und Cr Cagrephii Code boo.

D. If: to Impimnt this exremnt a contrut is ancored linto for thu periormance of sirvisos outsice the Unit.d Stares by U.S. fitizons, the Corps of Engineers will take approprist: actian to assur. compliance with the loyalty and jecurity investigation requircments of Section 111 of the Foroign Assistance ano Felat.d Agencies Arproprlation Act of $1966^{\circ}$ (Fublic Law 39-273).

\section{E, ADMINISTRATIVE ARRANG EMENTS}

1. "Projoct" as defined in this subparagraph "E" shall mian all necessary work in eonniction with the disign and construction of the Herat-Islam Gale road.

2. Provisions sit ferth in the bilatcrel agr ements (datod February 7,1951 ; Jine 30, 1953; June 9, 1954; Apri1 28, 1955; Juie 23, 1956; and any others cumently 


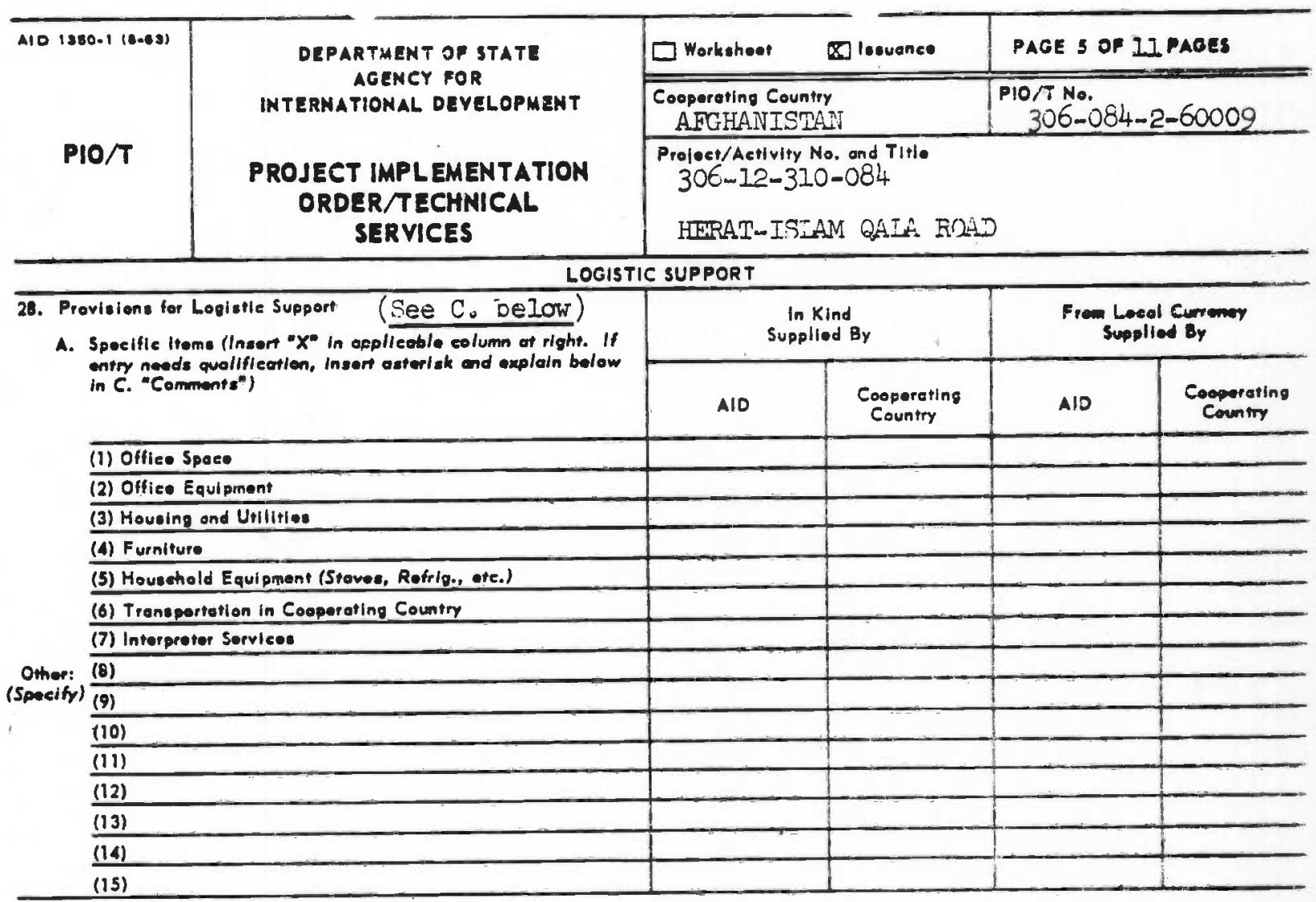

B. Additional Focilitios Available From Other Sources

\section{Commente}

The Corps of Engireers will provide housing, iccia tuansportiation and all other logistic support for its personnel. Financing of these eiements in Afghanistan outside of Kabul will be with ioun funds through arrangements with the construction contractor during coristimiction. 
CONIINUATION - PIO/T
Page 7 of 11

AFGHANISTAN $\quad 306-0,84-2-60009$

$306-12-310-084$

HERAT-ISIAM QAIA ROAD

\section{Block 21-B-(1) - Continued}

Use of third country nationals (TCN) employees by the Corps for services to be provided under Phase III Is rot authorlzed.

RGA has arreed to faclifiate the entry, movement and work of and and ali required personnel. Corps' personnel and all other personnel under contract to the corps are to be cleared by RGA which w1ll process all requests for cleararce expedit10isiy.

The sumary of FY-1966 estimated personnel requirements for the corps is shown on the PAJA Budget Plan (Appendix B). Since the services will continive in FY-ó? and on into FY-69, the Corps has provided, at $A D^{\prime} s$ request, an estimate of services reguirec in those years as well.

\section{BIock 24-E - Continued}

existing) between the Govermment of the U.S. anc. the RGA ano applicanif for the furpose of implementing this project.

3. The Corps, its Contractors and the Contractor'for the constraction of the Herat-Islam qala Road, with the approval of the Mliistry of Puislic hicks, may establish during the term of tiis p=oject such wage controls and pajment satsduies as, in their judgment, are reasonable and proper for the uccomplishment of this project. It is intended that wages of local employees be comensurate with the neture and conditions of the work and adequate to attract and hold needed labo: witiput distugting other projects.

4. The RGA has desigrated a Project Director who will act IOr PGA witis resrect to both techrieal and administrative aspecis of these armangmet $z$. It is contamiated that an agreement will be reached between the Corjs and tie RGA which rill ventil the duties and obligations of the paxtles with respect to the minses i... whan the Corps acts as the agent or representative of the XGA in tine ecmintation of the contract for the constriction of the road.

5. The United States, through fts deslgree, the lorps, will have the wight to select corporations, comparies, partmersips or indivaluais for the jurcose us linolementation of this project.

6. For the purpose of these arrangemerts, the word "deeignes" es slberuently ised shall include the Corps and its contractors and thelr sub-contractors and tils contractor for the construction of the Herat-Islam lala Road.

7. The facilities or services to ze furnished by RCi ir correction with the project shall include the followirg:

a. The necessary areas and access road as well as al-ways for ajncritt use, with rights of entry for the purpose of survey, investigation and consiruction, erd 
CONTINUATION - PIO/T
Page 9 of 11

AFGHANISTAN $\quad 305-0014=00039$

$306-12-310-034$

HERAT-ISLAM QALA RCSII

\section{Block 24-E - Continued}

property of the U.S. Government and 1ts designees and to be rewover from citapod of in Afghanistan, provided that any applicable duty, taxes end licences pees sherem on are paid by the purchaser, in the event of their sale or disposes in iffiniristan.

j. Permit, with approval of the Ministry of Communtcations, the estaplish-

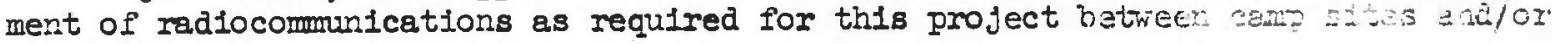
work sites.

k. Not require personnel of the U.S. Government or ite aesifnes tho chter Afghenistan for the performence of work under this project, to Fug lifecise or regis. tration fees to work in Afghenlstan. The RGA will normaily recelve L.5. arid thiracountry nationals employed by the U.S. Government or its designeec, and cleesed by RGA.

1. Provide the necessary adminlstrative organization itaticu a procedures to assure passage of all property, materials, supplies ana equirs in at 0.3 toms with a mirinum of delay, handling and storage time.

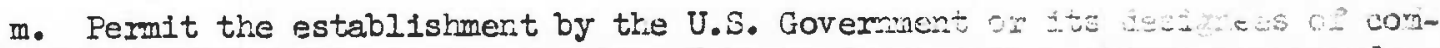
missary operations similar to the American Embassy Cooperative, available to all their employees, except those normally donicile? A and subject to eligiblity restractions that are not more levorab to thes controlling other American contract taams in Afginanistar.

n. Permit personnel employed by the U.S. Government crits wew or a holiday if they so choose.

o. Permit the establishment by the U.S. Goverment 0.7 . striction comps, messing ard other operations recessary for tie this project.

p. Not require the U.S. Govermment or its desigrees to profit taxes, licenses or registration fees to work oit tis pros resident representative in Afghanistan after completion of tz

q. (1) Hon-Afghan rationals who are employed in carnet. of this project, including their dependerts, Afghen income tax or other Afghan tax ârirg employment on this project.

(2) Such persons and members of their families dut $r_{2}$

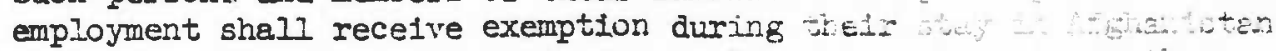
from the payments of import duties, seies, Iroperty of wis the

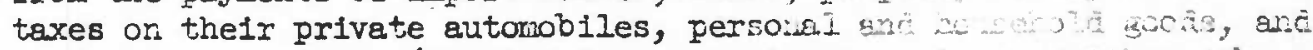
professional effects (IImited to professional egi wat jor requirements or recreational requirements) jroggh at the curctry 
CONTINUATION - PIO/T
Page 11 of 11

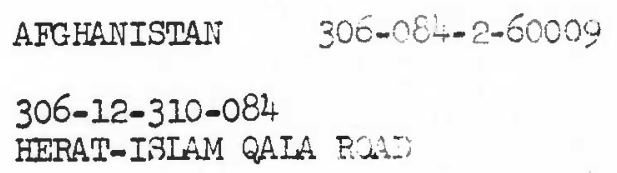

\section{Block 24-E - Continued}

v. Afghan personnel required by the Corps, or its designees, in the accomplishment of this Agreement w1ll be selected, hired and paid by the Copo, or its designees.

\section{Block $27-A$ - Cont1nued}

of the agreemert is attached as APPENDIX $C$ to the PASA.

The Corps of Engineers is responsible for implementation of the wry the the protect under the terms of the Particlpating Agency Service Agreement ( EACA) dated ATrd 4, 1963 , as this agreement may be amended. However, the Corps is respustole to the Director of USAID/A on program policy, project development and prodect changes; and on admilistrative matters, as defined in the PASA.

The Corps is responsible for the detalled technical and administrative direction of the operating activities of personnei to be provided for this rrich cors per-

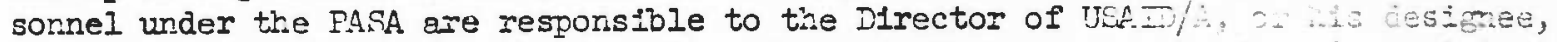
with regard to all contracts with $R G A$. In general, however, tis coma spectalists will be in continual direct contact with thels counterparts in Fin 
A P P E D D X XII .

TABULATION OF CONSTRUCTION

CONTRACT MODIFICATIONS

TABULATION OF FIELD CHANGES WITH

BACK-UP MEMORANDUMS FOR RECORD 
Provided for improvement and restoration of arainage causea by severe rainfall, and aacition of arainage facilities.

MOLIFICATION NC. 7 , dtd 23 JuI 67

Providea for air freight procurement of 72 pieces of kearing peds f'or Briage 96.5

MOLIFICATICN NC. 8, dta 17 May 67

Priviaea for the purchase and aelivery to the jobsite of 6,782 linear feet of different sizes ana gauges of currugated metal culvert pipes.

MOLIFICATICN NC. 9, dtd 3 Lec 67 $\$ 46,371.82$

Transport the GF equipment from Camp at Station 73 on HIC Highway to AIL Compouna in Kancahar. Leliver to the Government 20,500 pounds of joint sealer mace excess, aue to redesign of aip sectiuns. Procure briage railing in lieu of GF briage railing.

MOLIFICATICN NG. 1C, dtd 2 Feb 68 (Credit)

Credit for work not done or materials not utilized due to re-design.

MOLIFICATION NC. 11, dta $23 \mathrm{Feb} 68$ c. in

Install and transfer to the Government at Camp at Station 73 two 150-KW generators in lieu of one 350-KW generator as originally specified.

MOLIFICATICN NU. 12, ata 14 Nay 68 (Credit) $\$ 9,606.0 n$

Creait for an erroneousiy high price negotiated for asphalt, Portlana cement, and aiesel fuel. 


\section{APPENLIX XII \\ TABULATION UF FIELU CHANGES (No Cost Change)}

MEMORANLUM FGK THE RECORL, dtd 7 Lec 66

Leletion of Culvert vasonry Headwalis at Station $5+814$, $19.0 \mathrm{~N}$. Rt.

MEMCRANLUM FOR THE FECCIL, dEd 17 Sep 67

Changes to Lip Sections, Miscellaneous Earthwork and Culvert at Station $105+601$.

MEMCRANLUM FCR THE RECCRD, dta 12 0ct 67

Additional Signs and widening for Toll Station. 
MELITELT.ANEAN LIVISICN, U. S. CORPS CF ENGINEEFS

U. S. ENGINEEK AREA

AFGHAN]STAN

MLDFK

7 December 1966

MENOFANLUM FCH THL HECORL

SUBJECT: Leletion of Culvert Masonry Herawalls at Stations $5+814$ and $19.0 \mathrm{M} \mathrm{F:T}$.

Cn this aate, the undersignea, discussed with Mr. Josenh Kanaday, Jr., $\mathrm{HHC}-\mathrm{HIC}$, a possible credit to the Government by the aeletion of the subject Headwalls.

Mr. J. Kanaday mentioned that the following extra work aone at no cost to the Government should offset the credit for the deletion of headwalls, as listed:

1. Araitional excavation requirea to connect culvert inlet and outlet to invert of existing interceptor ditch.

2. Eackfill and restoration of that portion of the old road connecting to the new road which was cut during the construction of the new road.

3. Excavation and grading of swale line to aayli ht witches from aciational culverts at Staticns $0+841$ and $0+883$ across the road to mosque.

4. Cutting and refinishing (lining with bricks) holes of mua brick wall to connect culverts numbers 6,7 ana 8 to existing irrigation ditch inside the mud wall.

5. Mobilization and demobilization of equipment anc personnel to install acaitional culvert pipes at Station $2+014$ when operations wert alreaay 13 kilumeters ahead at the time of installation on 9 October 1966. 
MEMORANLUM! FCR THE I ECCED

7 Lecember 1966

SUBJECT: Leletion of Culvert Masonry Headwalls at Stations

$5+814$ and $19.0 \mathrm{M} \mathrm{KT}$

It was therefore agreed that the credit f or the deletion of culvert masonry heacwall at Station $5+814$ is a fair and reascnable comnensation for the above menticned extra work performed by tho Contractor. This agreement will not change any part of the contract such as cost or contract time.

\author{
/s/ Lonald E. Sells \\ $/ t /$ LCNALL E. SELIS \\ Major, Corps of Engineers \\ Actinf lirea Engineer
}

Concur:

/s/ Joseph Kanaaay, Jr.

$/ t /$ JOSEPH KANALAY, JR. Assistant Proiect lianager

Afghanistan Hi ghway Constructors - HTC 


\section{MHUITERRANEAN DIVISION, U. S. CORPS OF ENGINEERS \\ U. S. ENGINEER AREA \\ AFGH ANISTAN}

MDDF

17 September 1967

MENORAINDUM FOR THE RECORD

SUBJECT: HIQ Road

It was agreed by the undersigned on this date that the attached Scope of Work includes deletions and additions that are of equivalent value. That the deletion listed in Item $A$ is a fair and reasonable compensation for the additional work listed in Itams $A, B, C, D$ and E. That the work performed will be accepted as being equal or better than that cailed for in the plans and specifications. And that all items will be performed with no change in contract price or time of performance.

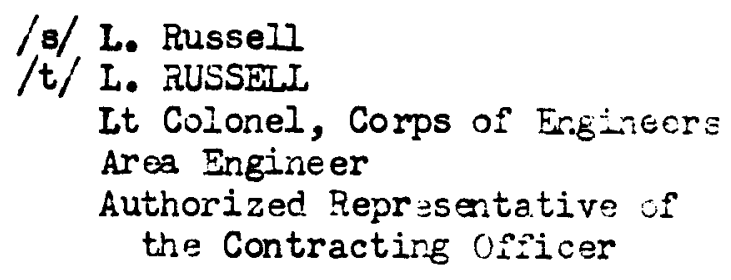

/s/ Joseph Kanaday, Ji.

$/ t /$ JOSEPE KiviviYY, JR.

Authorized Representative of

the Afghainistan Highway Constructors - HIQ 


\section{CONTRACT NO. (RGA) DACA-75-67-C-0005 \\ MISCEILANEOUS REVISIONS ON CUIVURT NO. 139 \\ DIP STATICN $34+604$ AND DIPS ADDED BY \\ REDESIGN WORK, HERAT-ISLAM QALA HIGHWAY}

\section{SCOPE OF WORK:}

The Contractor shall furnish all plants, labor, materials and equipment and perform all operations necessary for the miscellan zous revisions on Culvert No. 139 Dip $34 \pm 604$ and Dips added by Redesign Work, HIQ Highway.

\section{Description of Change:}

A. Delete 300 Iinear meters of Dip shoulder protection and loose riprap from Station. $33+190$ to station $33+4 \% 0$ on Dip $34 \pm 604$. Build up and compact to grade the shoulder area where the Dip protection was deleted. Extend the existing Interceptor Ditch up to Station 33+550. Raise grade at the end of Dip and transition to existing grade at Station $36+300$.

B. Raise grades on Dips added by.Redesign liork:

(1) Dip Station $64+450$ to Station 64+550: Raise end of Dip and transition to Station $64+250$.

(2) Dip Station $53+610$ to Station 53+670: Raise end of Dip and transition to station $53+700$.

(3) Dip Station 47+717 to Station 47+817: Raise end of Dip and transition to station $47+950$.

(4) Dip Station 46+880 to Station 46+960: Raise beginning of Dip and transition back to Station $46+700$.

(5) Dip Station 41+494 to Station 41+534: Raise ard of Dip and transition to Station $41+600$.

(6) Dip Station $26+197$ to Station 26+237: Rajse end of Dip and transition to Station $26+300$.

(7) Dip Station $22+184$ to Station $22+224$ : Raise end of Dip and transition to Station $22+275$.

(8) Dip Station 18+050 to Station 18+350: Raise end of Dip and transition to Station $18+425$.

(9) Dip Station $13+165$ to Station $13+185$ : Faise end of Dip and transition to Station $13+225$. 
(10) Dip Station 11+117 to Station 11+137: Haise end of Dip and transition to Station $11+200$.

(11) Dip Station 10+230 to Station 10+330: Raise end of Dip and transition to Station $10+400$.

(12) Dip Station 9+165 to Station 9+185: Kaise end of Dip and transition to Station $9+275$.

(13) Dip Station 8+690 to Station 8+710: Raise end of Dip and transition to Station $8+875$.

(14) Dip Station $8+238$ to Station $8+368$ : Raise end of Dip and transition to Station $8+600$.

C. Prepare subgrade and backfill with select borrow and subbase materials for 450 linear meters to receive concrete for a dip section between Station $17+600$ and $18+050$. Subsequently iuild up to grade using base overlay the same section to receive DEST roadway when the dip was deleted.

\section{Scarify, Elade, Compact and Final Dress:}

transition ends.

(I) The areas near the ends of the above Dips and

(2) The areas adjacent to beginnings and ends of Dips Station $46+030$ to Station $46+110$, Station $23+956$ to station $23+976$, Station $21+106$ to Station $21+126$, Station $19+855$ to Station $19+875$ and Station $19+204$ to Station $19+224$.

(3) Beginnings and ends of Dips $8 \pm 303$ and $10 \pm 280$ transition to existing grade when the concrete Dip pavement was changed to DBST Dip pavement. $E_{8}$ Relocate Culvert No. 139 to Station $205+601$, change
skew to $70^{R}$, substitute masonry headwalls for reinforced concrete,
place grouted riprap protections and excavate channel on hoth sides. Fill upstream original waterway with materials from new charnel.

2. Technical Specilications and Contract Performance Tire:

No change in Technical Specifications and Contract Performarce time will be allowed by reason of this change. 
MEDITERRANLAN LIVISION, U. S. COFPS OF ENCINEIIIS

U. S. ENGINEER AREA

AFGHANISTAN

MEMORANUUM FOF THE FFCCRD

12 Cctober 1967

SUBJECT: Contract No. (RGA) LACA-75-67-C-0005, Miscellaneous

Acicitional Work, Herat-Islam Cala Highway

1. It was agreed by the undersigned on this aate that the work contained in the attached scone of Work would be accomplished and paid accoraing to the following payment scheaule:

a. All items identifiable as normal contract line items at the contract unit cost. This includes scope of work items $B, E$ and $F$.

b. Cther items acroroing to the following scheaule:

1. Vasonry wall monuments, 2 each, $\$ 250.00$ each $\$ 501.00$

2. Kilometer posts, 13 each, $\$ 50.00$ each $\$ 650.00$

c. All guide posts replaced to date and reinforcing picec in guiae nosts was at no cost to the Government.

2. Payment for this additional work will be made under tre applicable line item or as grouted riprap for the monuments (line item 1C) ana as reinforce concrete (line item 12) for the kilometer posts.

1 Incl as /s/ L. Russell

$/ t /$ L. RUSSELL

Lt Colonel, Corps of Enginers Area Engineer Authorized Representative of the Contracting Officer

$/ s /$ James A. Sumner

$/ t /$ JAMES A. SUMNEK

Authorized Representative of the

Afghanistan Highway Constructors - HIQ 


\section{SCOPE OF WORK:}

The Contracotr shall furnish all plants, labor, materials and eouipment and perform all operation necessary f'or the miscellaneous adaitional work on the Herat-Islam Qala Highway.

1. Lescription of Change:

A. Reintorcing of guice osts to ve installed from Station $110+270$ to the ena of the road and those to be replaced, shown as deficiency on LL Form 1354 dated 14 September 1967. Use scrap steel reinforcement generatea from Government-Fumished Property and fill holes with Class "X" concrete.

B. Construct and install three (3) road sign pasts for toll house at approximately Station 36+016 \pm , Station $36+40 \mathrm{C} \pm$ and Station $113+400 \pm$, two (2) road sign posts for police barrier at approximately

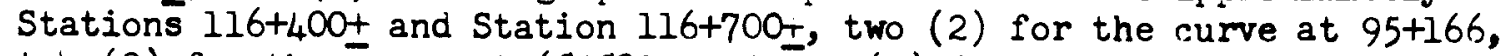
two (2) for the curve at $68+839$, and two (2) for the dip section at Station $47 \pm 767$, one (1) for end of road at Islam Qala at Station $122+725$.

C. Construct and install thirteen (13) kilometer pests, at an interval of 10 kilometers, starting from the Iranian border.

L. Construct two (2) cement rubble masonry walls for monument at a proximaterly Station $1+000 \pm$ and Station $116+300 \pm$ using faced stones excess from culvert heacwalls. The inscribed marble panels and ironze plaaues will be furnished by FGA.

E. Widen road nism and pavement between Station $113+530.58$ and Station $113+592.50$ and at Station $36+200$ (one siue only) tor toll houses.

F. Wicen shoulaer using subgrace materials from Station $113+713.14$ to Station $114+00$ ana from Station $114+037.50$ to $114+250$ (right side cnly) for parking apron at the custom area.

2. Adcea Lrawin s:

Lrawing No.

HIQ-SK-10

HIQ-SK-II

HIQ-SK-12

HIQ-SK-13 $\underline{\text { bescriotion }}$

Wicening of Roc.d Prism and Pavement

wioening of Shoulder

Kilometer Post

Masonry Wall for Monument
Late

25 September 1:67

25 Sept ember 1967

2. Cctober 1967

2 October 1967 
3. Techinical Specifications and Contract Performance Time:

No change in Technical Specifications and Contract Performance Time will be allowed by reason of this change. 
\begin{tabular}{l} 
A P P E N D I X XI \\
\hline
\end{tabular}

CORPS OF ENGINEERS STAFFING TABLE 


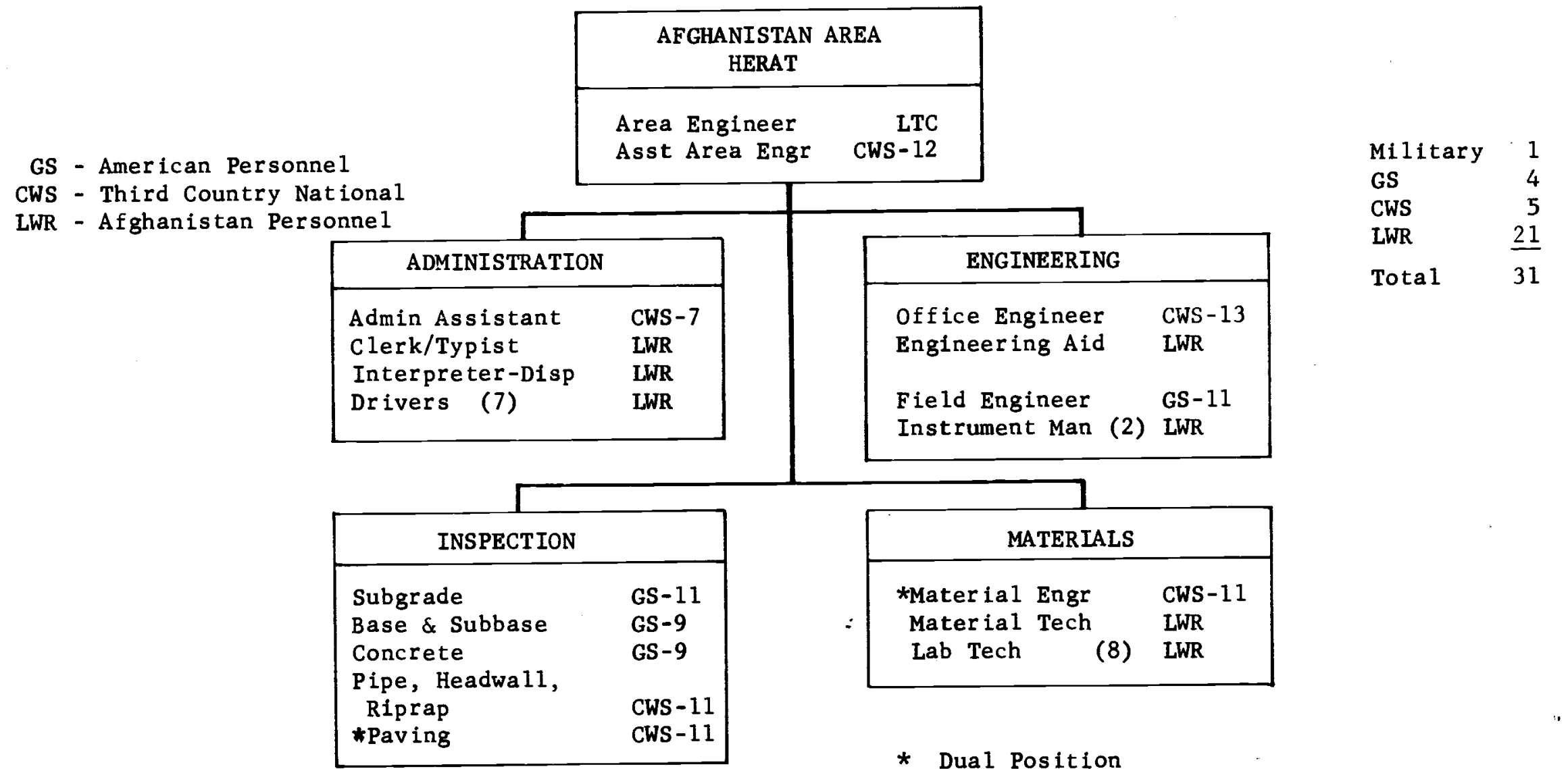

STAFFING $1966-1967$ 


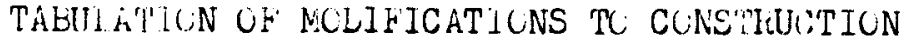 \\ CWNTFiACT (RGA) LACAA-75-67-6;-0005 \\ HEKAT-ISIAM GALA HIGHWAY PKCJICT}

MOLIFICATICN NS. 1, ata 31 Lec 66

Provicied for the contractor to furnish, service and maintain for the Government use eight (8) vehicles, four (4) of which equipped with communication type raaio; for the celetion of 500 square feet of the Government laboratory facility floor space. Also provice for revision of culvert pipe headwalis.

MOLIFICATICN NO. 2, dtd 6 Jan 67

Provided for widening asphalt pavement from 5.50 to 6.20 meters and maintain the road crown of $2 \%$ t $:$ the edge of pavement. Lelete. 241.71 linear meters of karese tunnel lining.

NOLIFICATION NC. 3, ata 23 Jan 67

Provided for the contractor to furnish 2 additional vehicles for Government use; service, repair ana maintain for an estimated 75,000 miles.

MOLIFICATICN NC. 4, dta 7 Feb 67

$\$ 900.00$

Proviced for loading, transporting ana unloading 1,529 linear feet of different sizes and gauges of Government owned corrugated metal nipe from Kanciahar to Herat jobsite.

lCLIFIChTICN NO. 5, dtd 9 Apr 67

Provided for substitution of two (2) $66 " \varnothing$ GF corrugatea metal pipes tor $2 \times 2$ meters of reinforcea concrete box culvert at Station $105+664$. 
A P P E N D I X X

GOVERMMENT-FURNISHED PROPERTY 


\section{APPENLIX X}

\section{GCVERNRENTT-FUKLNISHEL PHIOPERTY}

Following is a list of Government-furnished property which was located in the Government storage yard at Kandahar for use by Contractor:

a. Culvert Pipes and Accessories:

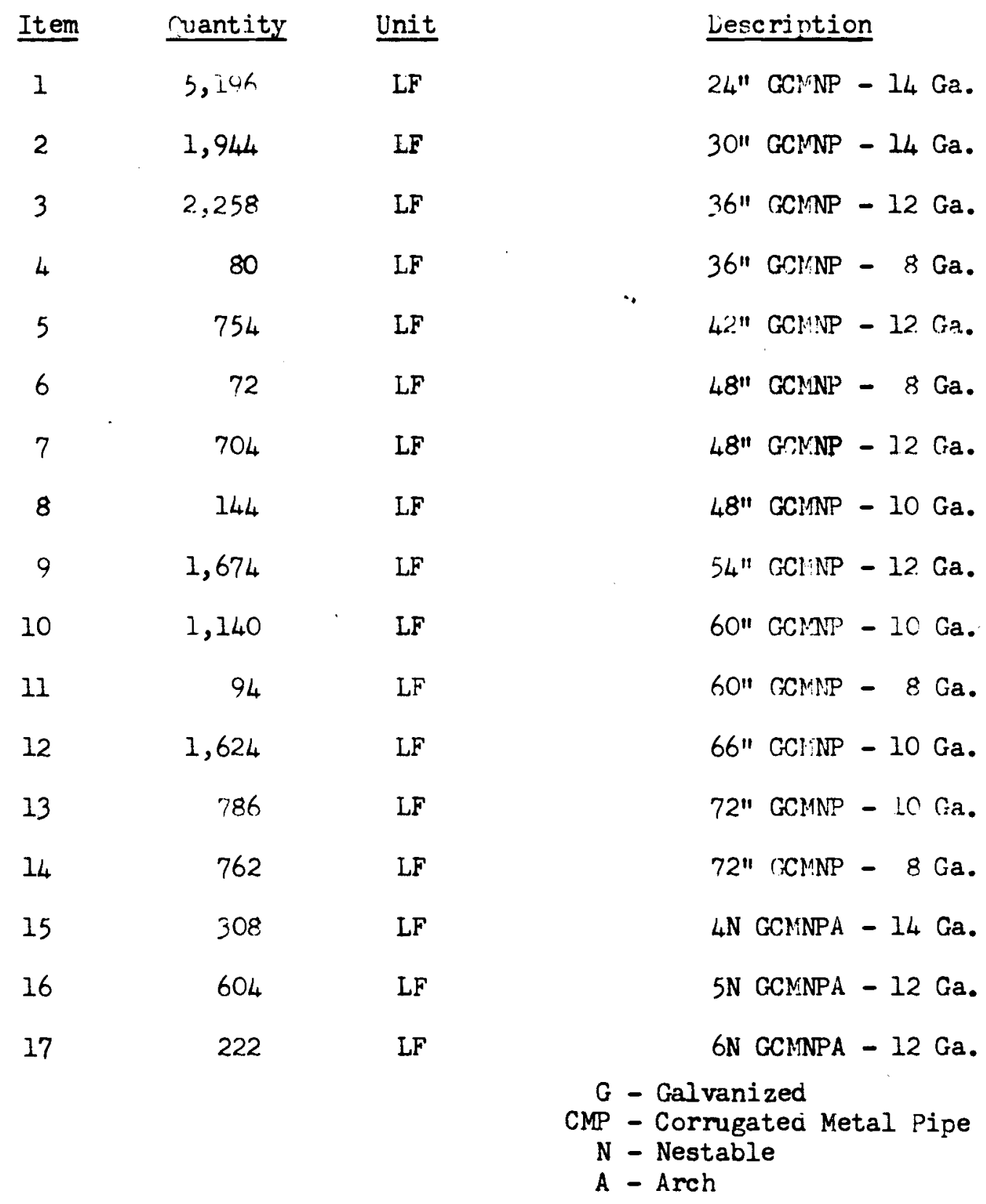


Culvert Pipes and Accessors (Cont'd)

\begin{tabular}{|c|c|c|c|}
\hline Item & Quantity & Unit & Description \\
\hline 18 & 287 & LF & 7N GCMNPA - 12 Ga. \\
\hline 19 & 126 & $\mathrm{LF}$ & 7N GCMNPA - $10 \mathrm{Ga}$. \\
\hline 20 & 396 & $\mathrm{LF}$ & $8 N$ GCMNPA - 12 Ga. \\
\hline 21 & 214 & $\mathrm{LF}$ & 8N GCMNPA - $10 \mathrm{Ga}$. \\
\hline 22 & 344 & $L F$ & $9 \mathrm{~N}$ GCMNPA - 12 Ga. \\
\hline 23 & 76 & LF & 9N GCMNPA - $10 \mathrm{Ga}$. \\
\hline 24 & 286 & $\mathrm{LF}$ & ION GCMNPA - $10 \mathrm{Ga}$. \\
\hline 25 & 56 & $\mathrm{LF}$ & ION GCMNA - $8 \mathrm{Ca}$. \\
\hline 26 & 1 & $\mathrm{LF}$ & 144" Structure-8 Ga \\
\hline
\end{tabular}

b. Bridge Materials and Accessories:

\begin{tabular}{|c|c|c|c|}
\hline Item & Quantity & Unit & Lescription \\
\hline 1 & 72 & Ea & $\begin{array}{l}\text { Neoprene Bearing Pads } 20 \mathrm{~cm} . \times 4 \mathrm{~cm} \text {. } \\
\times 39 \mathrm{~cm} \text {. }\end{array}$ \\
\hline 2 & 482 & Ea & Copper Stirrups \\
\hline 3 & 640 & Ea & 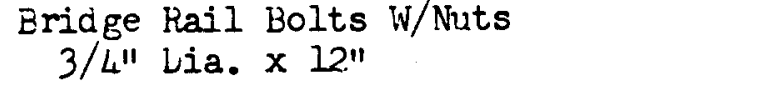 \\
\hline 4 & 612.83 & LF & 6" WF Beam for Bridge kail Posts \\
\hline 5 & 134,966 & LB & RE-Steel No. Il Bar \\
\hline 6 & $1,674.75$ & LF & $\begin{array}{l}\text { 3" Lia. Extra Heavy Pipe for Bridge } \\
\text { Rail }\end{array}$ \\
\hline 7 & 14 & $\mathrm{Ea}$ & $\begin{array}{l}\text { Neoprene Bearing Paas } \\
25 \mathrm{~cm} \cdot \times 5 \mathrm{~cm} \cdot \times 40 \mathrm{~cm} \text {. }\end{array}$ \\
\hline 8 & 8 & Ea & $\begin{array}{l}\text { Diaphragm Rods w/o Nuts } \\
\text { I" Lia. } \times 22^{\prime} 2^{\prime \prime}\end{array}$ \\
\hline
\end{tabular}




\begin{tabular}{|c|c|c|c|}
\hline Item & Quantity & Unit & Description \\
\hline 9 & 8 & $\mathrm{Ea}$ & $\begin{array}{l}\text { Diaphragm Rods w/o Nuts } \\
\text { l" Lia. } \times 22 \text { 'll" }\end{array}$ \\
\hline 10 & 32 & $\mathrm{Ea}$ & Cast Iron Ogie Washers \\
\hline 11 & 32 & $\mathrm{Ea}$ & 60 MM Fabreeka Washers \\
\hline 12 & 32 & Ea & Diaphragm Rod Nuts l" \\
\hline 13 & 96 & Ea & $12 \mathrm{~cm} . \varnothing \times 1 \mathrm{~cm}$. Plate Washers \\
\hline 14 & 78 & LF & $\begin{array}{l}\text { 2-1/2" Lia. Extra Heavy Pipe for } \\
\text { Bridge Rail }\end{array}$ \\
\hline
\end{tabular}

c. Eouinment

\begin{tabular}{|c|c|c|c|c|}
\hline Item & Qty \& Unit & Type of Equipment . & Make & Model \\
\hline 1 & $6 \mathrm{Ea}$ & Trucks Cargo $2 \frac{1}{2}$ Tons & Studebaker & $1:-35$ \\
\hline 2 & $14 \mathrm{Ea}$ & $\begin{array}{l}\text { Gracers, Notor, with } \\
\text { Scarifiers }\end{array}$ & Galion & 11.8 \\
\hline 3 & $10 \mathrm{Ea}$ & $\begin{array}{l}\text { Scrapers Earth Moving, } \\
\text { Towed, } 4 \text { Rubuer whl Mtd }\end{array}$ & Le Turneau & LP \\
\hline 4 & $23 \mathrm{Ea}$ & $\begin{array}{l}\text { Tractor Crawlers w/Lozer } \\
\text { Blade }\end{array}$ & I. H. C. & $T D-24$ \\
\hline 5 & $5 \mathrm{Ea}$ & $\begin{array}{l}\text { Air Compressors } 500 \text { CFM } \\
\text { Trailer Mtd Pneu Tires }\end{array}$ & Leroi & $500 \mathrm{N2C}$ \\
\hline 6 & $1 \mathrm{Ea}$ & $\begin{array}{l}\text { Air Compressor } 315 \mathrm{CFN} \\
\text { Trailer Mtd Tires }\end{array}$ & $\begin{array}{l}\text { Ingersoll } \\
\text { Rand }\end{array}$ & $I E-315$ \\
\hline 7 & $6 \mathrm{Ea}$ & $\begin{array}{l}\text { Crane Power Unit Rvg } 28 \frac{1}{2} \\
\text { Ton Crawler }\end{array}$ & Harnischfeger & r $655 \mathrm{~A}$ \\
\hline 8 & $1 \mathrm{Ea}$ & $\begin{array}{l}\text { Shovel Front } 1 \frac{1}{2} \text { cu. yd. } \\
\text { for Crane } 655 \mathrm{~A}\end{array}$ & $P \& H$ & $655 \mathrm{~A}$ \\
\hline 9 & $2 \mathrm{Ea}$ & $\begin{array}{l}\text { Crushers Roll Type G.E.L. } \\
\text { Trl Mtd Steel Wheels w/ } \\
\text { Screens and Feeder }\end{array}$ & Pioneer & 42-VA \\
\hline
\end{tabular}


c. Equipment (Cont'd )

Item Qty \& Unit Type of Ecuipment Make Moael

$102 \mathrm{Ea} \quad$ Crushers Jaw Type G.E.L. Crawler Mta with Screens and Feeder

Pioneer $\quad 56 \mathrm{~A}$

$116 \mathrm{Ea} \quad$ Crusher and Screening Plant Iowa $\quad$ L.J. 50

12. $6 \mathrm{Fa} \quad$ Sc rew Lehyarator Towed Type Tyres 2 Mtd

Cedar Rapias 2018-E(1833)

$13 \quad 3$ Ea Concrete Bucket Bottom Lump

$3 / 4$ cu. yd Blaw knox

C.22:51

$14 \quad 1 \mathrm{Ea} \quad$ Lrill Star, Skid litd w/

Components

Speed Star 71SK

$15 \quad 2$ Sets Rock Cuarry Eçuipment, Approx.

30 Boxes Issued as Sets Ingersol Rand $x-71 \mathrm{wL}$

WFN 3 wgn

$163 \mathrm{Ea} \quad$ Backhoe Fronts w/Lipners

$17 \quad$ I Ea Nultifoote Paver, Single Lrum Multifoote $34-\mathrm{E}$

$187 \mathrm{Ea}$ Boom Section Length-10', W-3'6", $\mathrm{H}=3$ ! $-10^{n}$

$19 \quad 13$ Fia

Boom Section Length $20^{\prime}$, W-3'6', H-3'-10"

$20 \quad 9 \mathrm{Ea}$

Boom Section Length $05^{\prime}$,

W-3'6", H-3'-10"

$21 \quad 10 \mathrm{Ea}$

Reel-fueling Hose $40 \mathrm{Gal}$.

PPN, I $\frac{1}{2}$ " Lia. 50 ' Long, F-3

22

$3 \mathrm{Ea}$

Ambulance $3 / 4$ Ton $4 \times 4$

Lodge

23

$3 \mathrm{Ea}$

Conveyor Portable w/o Notors Cedar Rapids $30 \times 50$

24

$3 \mathrm{Ea}$

Conveyor, Portable w/elec.

Motor

Cedar Kapids 3035

25

$6 \mathrm{Ea}$

Lump Truck

International 200

26

$2 \mathrm{Ea}$

Self-Propelled Roller

Buffalo-Springfield 
c. Eouipment (Cont'd)

\begin{tabular}{|c|c|c|c|}
\hline Item & Cty \& Unit & Type of Fouipment & Make \\
\hline 27 & $1 \mathrm{Ea}$ & Tandem Roller & $\begin{array}{l}\text { Buffalo- } \\
\text { Springfield }\end{array}$ \\
\hline 28 & $2 \mathrm{Ea}$ & $\begin{array}{l}\text { Scale, Platform, } 30 \times 42 \text {, } \\
300 \text { lb. cap., } 2 \text { lb. } \\
\text { gradation }\end{array}$ & Winslow \\
\hline 29 & $580 \mathrm{LF}$ & $\begin{array}{l}\text { Forms, Concrete hoad, } \\
\text { with. } 7 / 8 \text { " pins, } 6 " \text { high, } \\
10 \text { foot sections }\end{array}$ & Blaw-Knox \\
\hline
\end{tabular}


A P P E N D I X IX

SELECTIVE METEOROLOGICAL DATA 


\section{APPENDIX IX}

\section{SELECTIVE METEOROLOGICAL DATA FOR HERAT}

\section{Job Temperature and Wind Velocity Record}

Temperature (Average Daily)

Month Min Max

1966

Sep $\quad 61.96^{\circ} \mathrm{F} \quad 96.03^{\circ} \mathrm{F}$

Oct $\quad 49.48^{\circ} \mathrm{F} \quad 83.45^{\circ} \mathrm{F}$

Nov $\quad 32.07^{\circ} \mathrm{F} \quad 65.51^{\circ} \mathrm{F}$

Dec $\quad 27.70^{\circ} \mathrm{F} \quad 63.87^{\circ} \mathrm{F}$

1967

Jan $24.21^{\circ} \mathrm{F} \quad 53.89^{\circ} \mathrm{F}$

Feb $\quad 28.85^{\circ} \mathrm{F} \quad 53.99^{\circ} \mathrm{F}$

Mar $\quad 38.68^{\circ} \mathrm{F} \quad 69.80^{\circ} \mathrm{F}$

Apr $\quad 49.16^{\circ} \mathrm{F} \quad 78.08^{\circ} \mathrm{F}$

May $\quad 56.68^{\circ} \mathrm{F} \quad 90.41^{\circ} \mathrm{F}$

Jun $\quad 64.21^{\circ} \mathrm{F} \quad 96.80^{\circ} \mathrm{F}$

Jul $\quad 70.90^{\circ} \mathrm{F} \quad 99.99^{\circ} \mathrm{F}$

Aug $\quad 68.70^{\circ} \mathrm{F} \quad 96.68^{\circ} \mathrm{F}$

Sep $\quad 58.88^{\circ} \mathrm{F} \quad 91.94^{\circ} \mathrm{F}$

Oct $\quad 45.00^{\circ} \mathrm{F} \quad 79.85^{\circ} \mathrm{F}$

$32.00^{\circ} \mathrm{F}$

$98.60^{\circ} \mathrm{F}$

Nov $\quad 40.59^{\circ} \mathrm{F} \quad 74.85^{\circ} \mathrm{F}$
Range

Low $\mathrm{High}$

$37.40^{\circ} \mathrm{F} \quad 105.80^{\circ} \mathrm{F}$

$82.40^{\circ} \mathrm{F}$

$77.00^{\circ} \mathrm{F}$

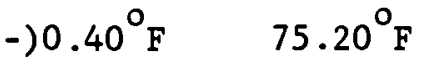

$12.20^{\circ} \mathrm{F}$

$77.00^{\circ} \mathrm{F}$

$21.20^{\circ} \mathrm{F}$

$87.80^{\circ} \mathrm{F}$

$26.60^{\circ} \mathrm{F} \quad 100.40^{\circ} \mathrm{F}$

$44.60^{\circ} \mathrm{F} \quad 114.80^{\circ} \mathrm{F}$

$51.80^{\circ} \mathrm{F} \quad 114.80^{\circ} \mathrm{F}$

$60.80^{\circ} \mathrm{F} \quad 111.20^{\circ} \mathrm{F}$

$59.00^{\circ} \mathrm{F} \quad 105.80^{\circ} \mathrm{F}$

$42.80^{\circ} \mathrm{F} \quad 107.60^{\circ} \mathrm{F}$
$8.32 \mathrm{MPH}$

$7.61 \mathrm{MPH}$

$13.91 \mathrm{MPH}$

$8.13 \mathrm{MPH}$
Wind Velocity (Average)

High

$22 \mathrm{MPH}$

$25 \mathrm{MPH}$

$25 \mathrm{MPH}$

$60 \mathrm{MPH}$

8.63 MPH

$15 \mathrm{MPH}$

10.10 MPH

$25 \mathrm{MPH}$

$10.68 \mathrm{MPH}$

$22 \mathrm{MPH}$

$9.96 \mathrm{MPH}$

$30 \mathrm{MPH}$

$10.83 \mathrm{MPH}$

$25 \mathrm{MPH}$

$13.86 \mathrm{MPH}$

$25 \mathrm{MPH}$

$13.45 \mathrm{MPH}$

$40 \mathrm{MPH}$

$11.67 \mathrm{MPH}$

$30 \mathrm{MPH}$

9.50 $\mathrm{MPH}$

(Est)

$25 \mathrm{MPH}$

(Est)

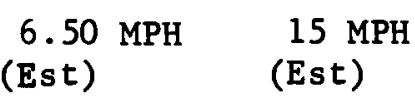

$6.50 \mathrm{MPH}$ (Est) 


\section{APPENDIX IX \\ JOB PRECIPITATION RECORD}

\begin{tabular}{|c|c|c|c|c|c|}
\hline \multirow{2}{*}{$\begin{array}{l}\text { Month } \\
1966\end{array}$} & \multicolumn{2}{|c|}{ Days of } & \multicolumn{3}{|c|}{ Highest in a Day } \\
\hline & & & & & \\
\hline August & - & - & - & & - \\
\hline September & - & - & - & & - \\
\hline October & 2 & - & Trace & & - \\
\hline November & - & - & - & & - \\
\hline December & 2 & - & 0.005 & Inch & - \\
\hline 1967 & & & & $\because$ & \\
\hline January & - & 2 & - & & 3.0 Inct \\
\hline February & 4 & 2 & 0.42 & Inch & $3.75^{\prime \prime}$ \\
\hline March & 6 & - & 0.39 & $"$ & - \\
\hline April & 10 & - & 0.52 & $"$ & - \\
\hline May & 1 & - & 0.21 & " & - \\
\hline June & - & - & - & & - \\
\hline July & - & - & - & & - \\
\hline August & - & - & - & & - \\
\hline September & - & - & - & & - \\
\hline October & 2 & - & 0.10 & $"$ & - \\
\hline November & 2 & - & Trace & & - \\
\hline
\end{tabular}


APPENDIX IX

SUMMARY OF AVAILABLE WEATHER DATA AT HERAT FOR YEARS 1958 THRU 1964

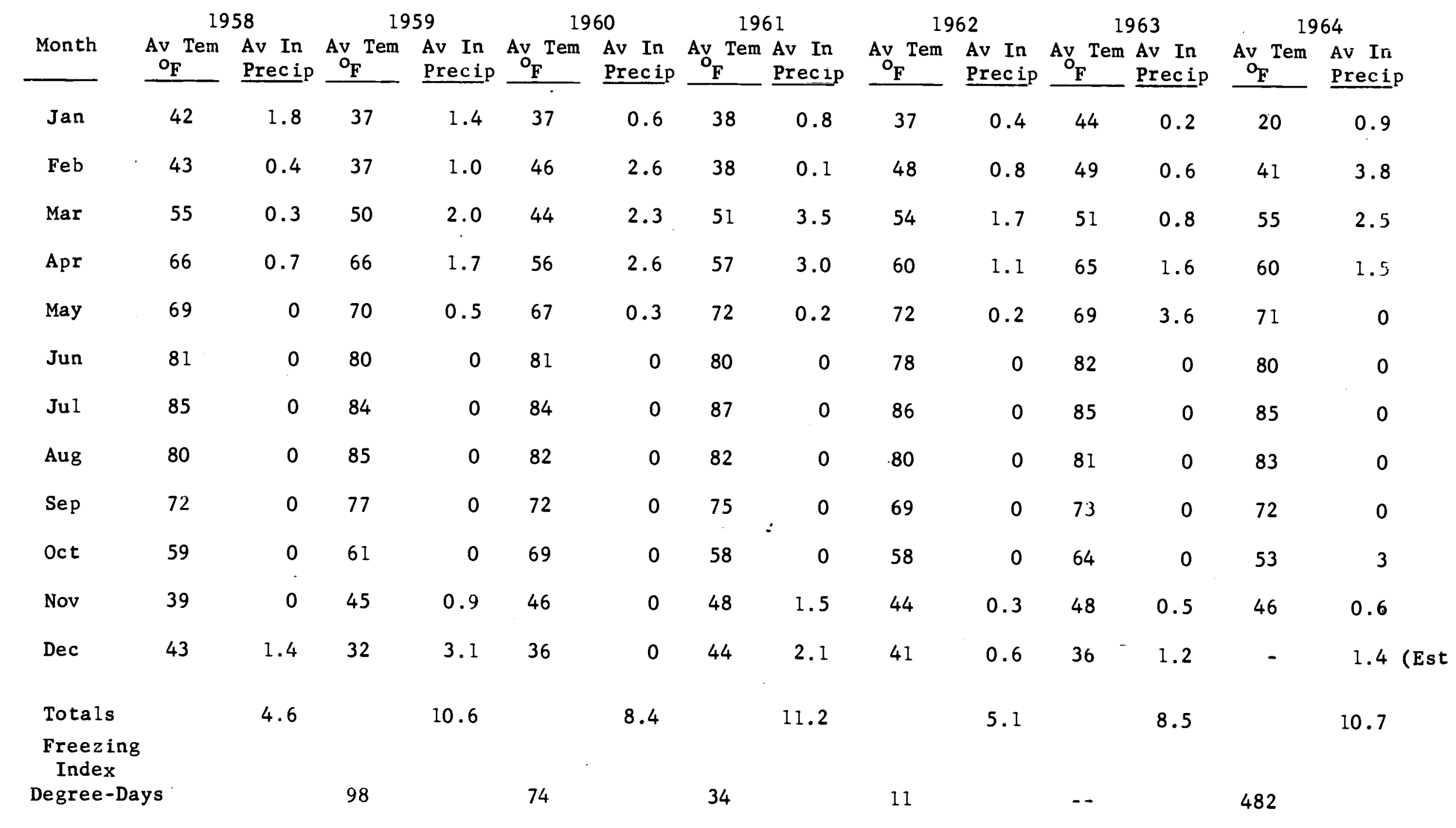


$\begin{array}{llllll}\text { A P P E N D I X VIII } & \end{array}$

TYPICAL ROAD SECTIONS

TYPICAL BRIDGE SECTION - ELEVATION

TYPICAL DIP SECTIONS 

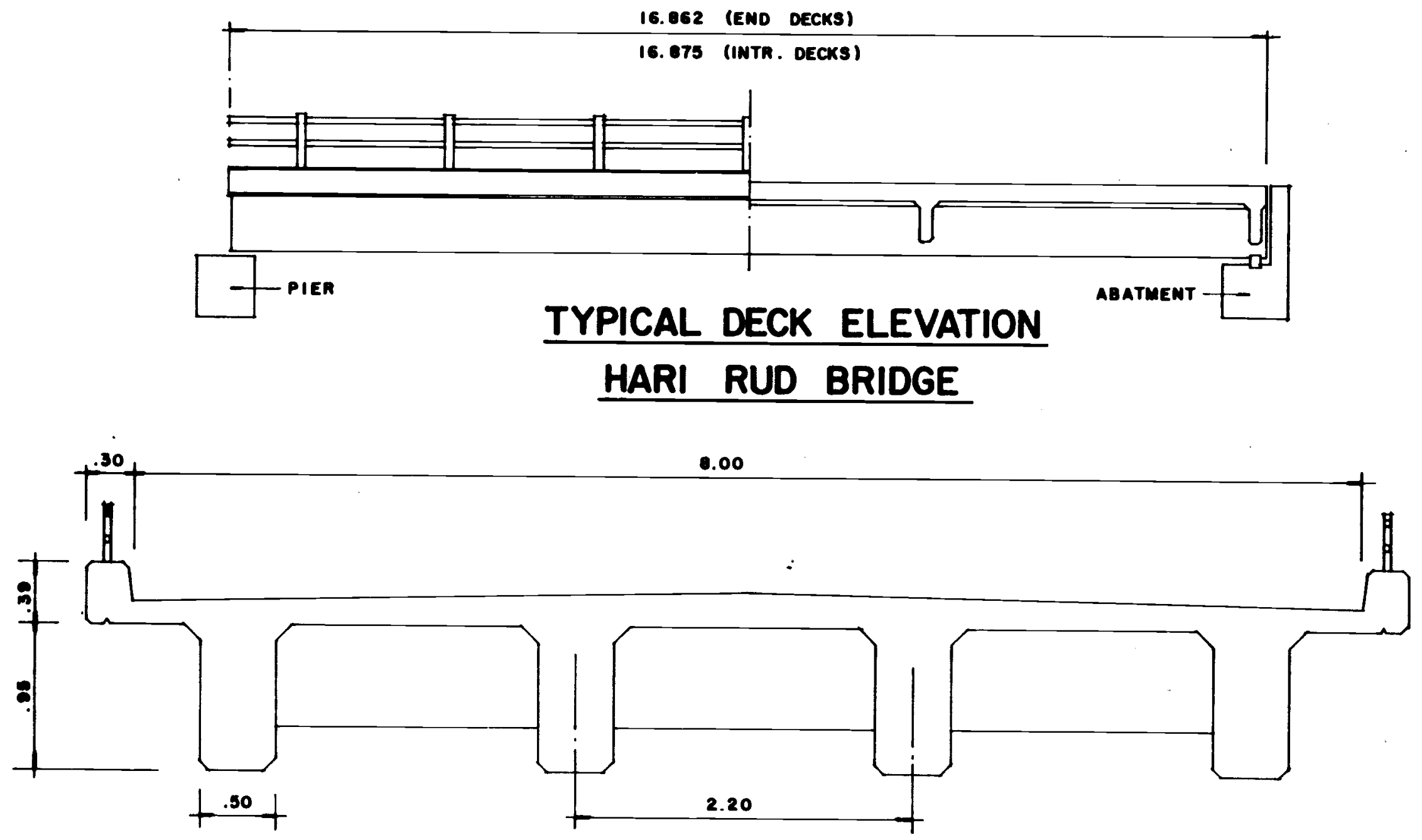

TYPICAL DECK SECTION

HARI RUD BRIDGE 


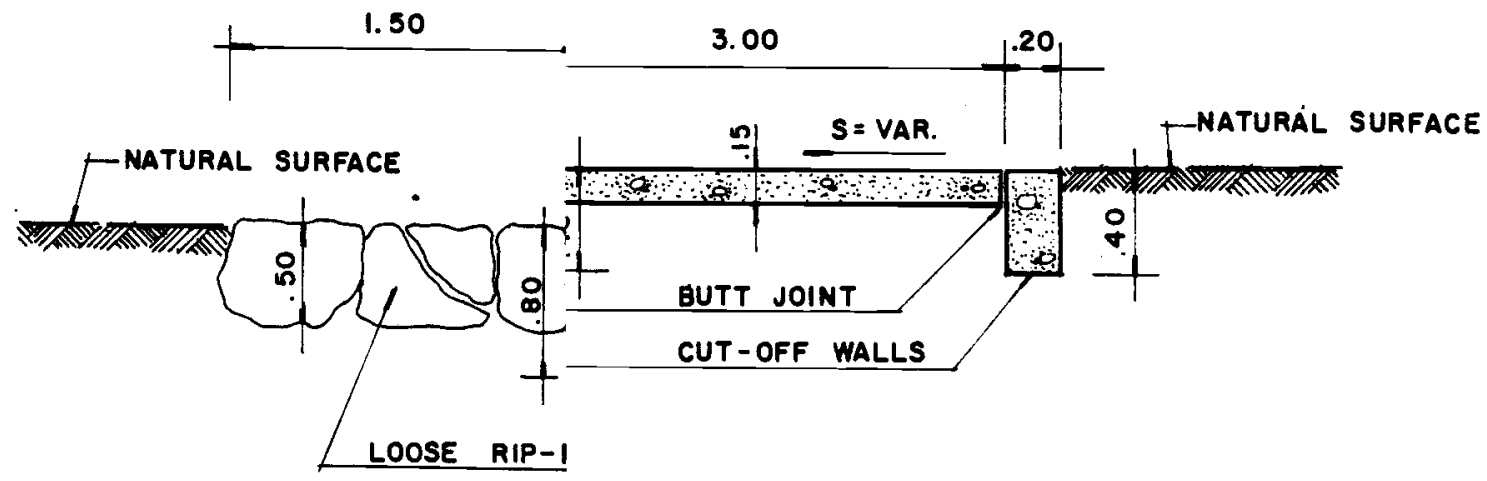

ST - SUSCEPTIBLE

\% \% CE-55 MAX. DENSITY

CE-55 MAX. DENSITY

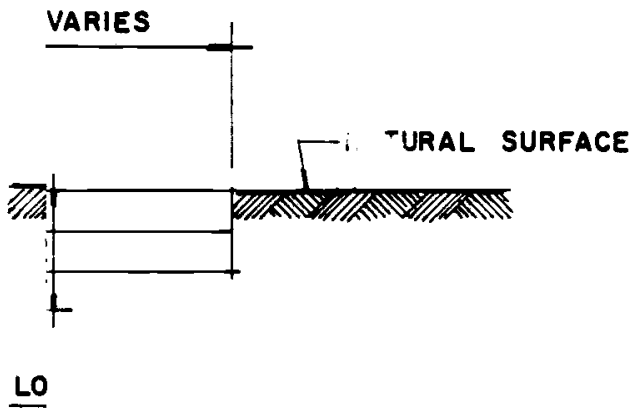




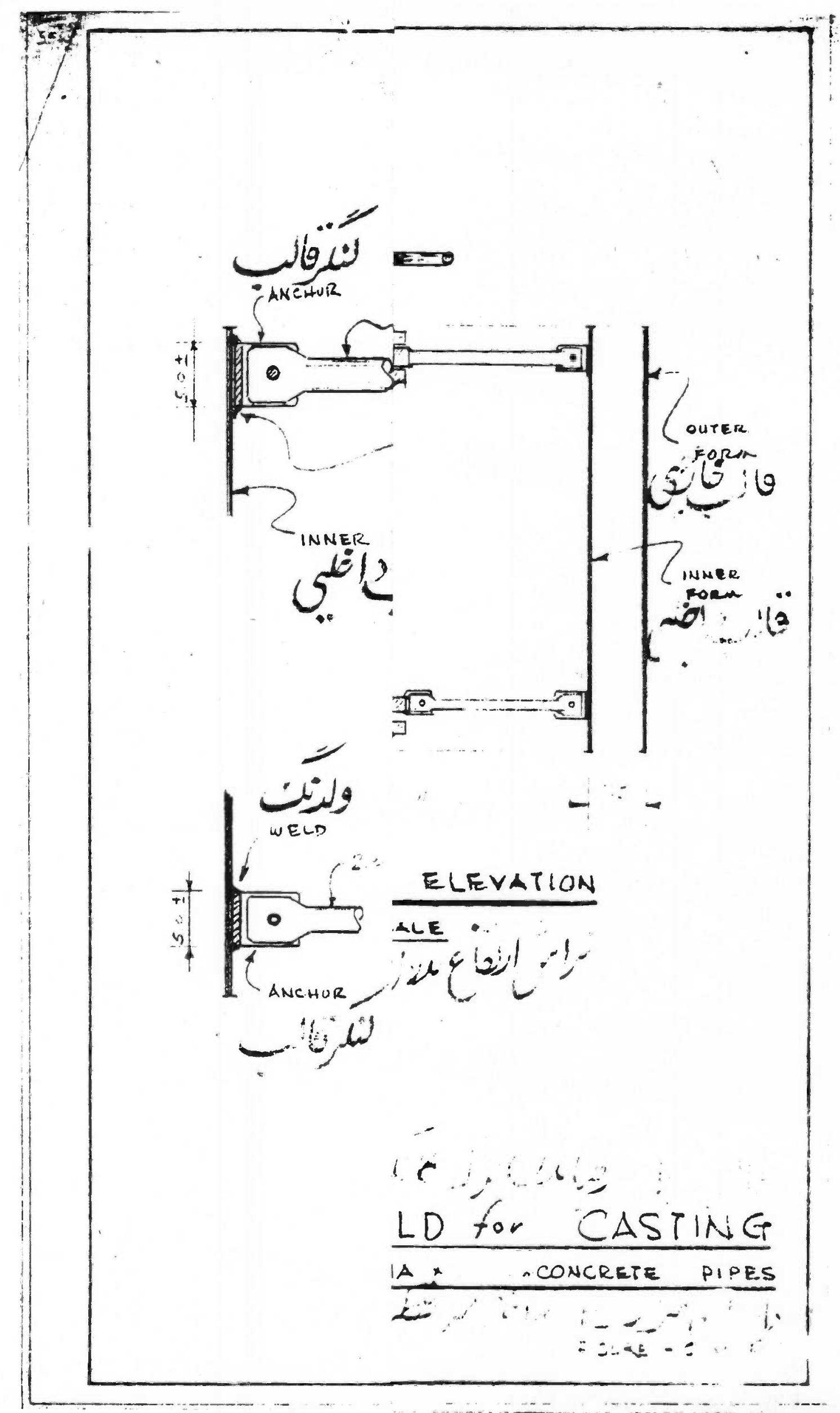




\section{MAINTENANCE ORCANIZATION FOR HIQ ROAD}

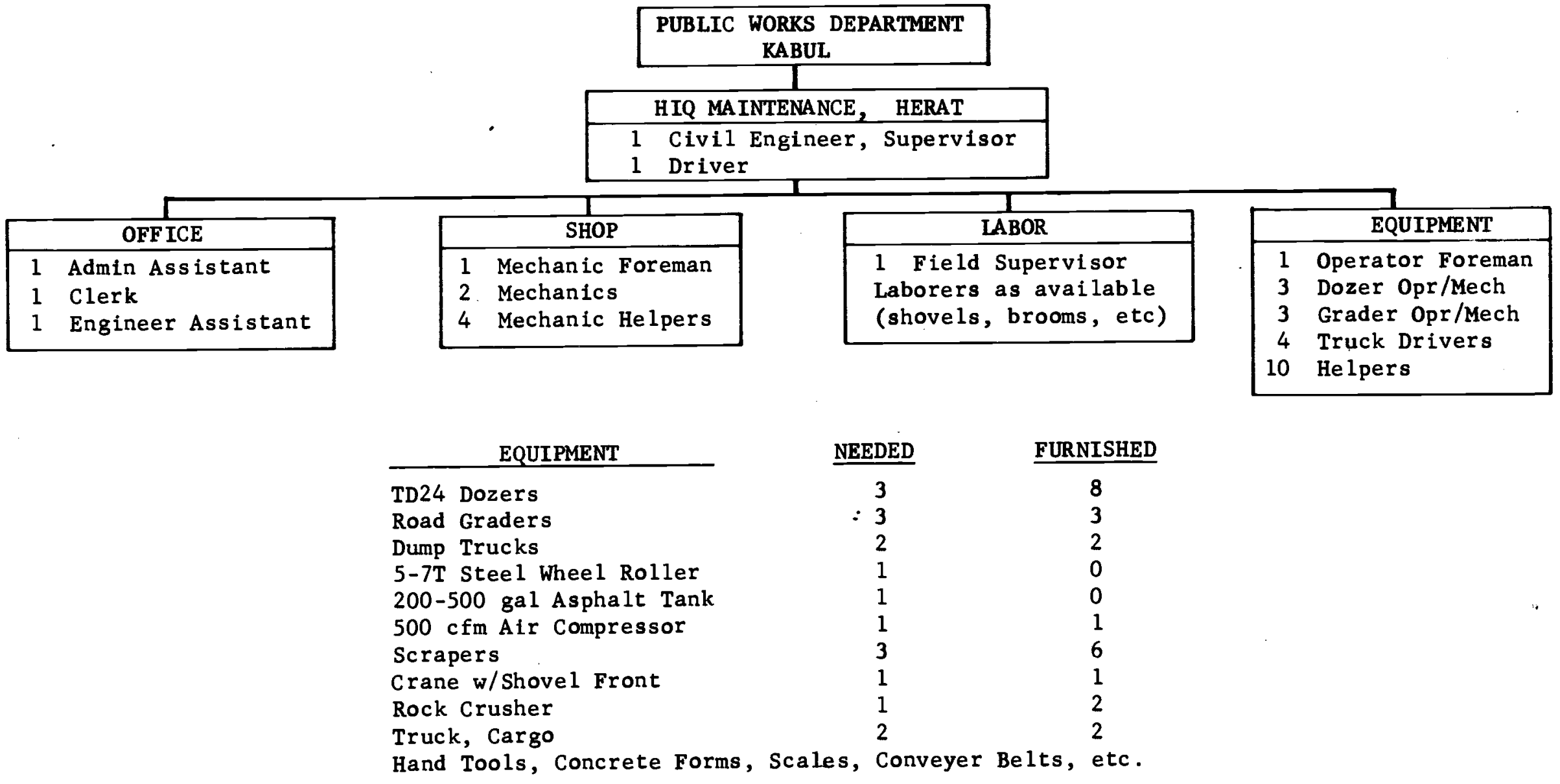


A P P E D D X XIII *

FINDING OF FACT CONCERNING

RAINFALL AND RUNOFF DAMAGE, APRIL - MAY 1967 


\section{MAINTENANCE OF SHOULDERS, EMBANKMENTS, "V" DITCHES AND BACK SLOPE}

Except for the limited use of patrol graders, this type of maintenance will have to be accomplished by hand work. Erosion, soil conditions, prevailing winds and silting again enter into the picture, and this type of repair and maintenance work will have to be a year-round or continuous operation. The " $V$ " ditches must be kept clean and open. Drainage must be checked. Grades and elevations of the " $V$ " ditches must be maintained so that no water stands along the shoulder upon the surface of the road. If water is allowed to stand for any period of time on the shoulders or surface of the road, the base will become saturated, pumping sections and base failures will appear in the traveled way. If these conditions are not corrected at once, the sections involved will have to be removed to a depth where the materials have sufficient compaction and stability, backfilled, compacted and resurfaced. Shoulders and embankments will be subject to erosion by wind and rains. The eroded areas must be backfilled and compacted before damage to the paved surface occurs. Laborers with shovels and hand tampers can accomplish this type of repair. Back slopes will require periodic maintenance. Here again, erosion by wind and surface water will damage the back slopes and fill up the "V" ditches. This also will be repaired by the laborers with hand tools; all that is required is to clean up the extra silt and debris so that there is a clean surface as initially built.

\section{MAINTENANCE OF DIP SECTIONS AND GROUTED RIPRAP DIKES}

Three different designs for dip sections were used during the construction of this project and maintenance problems will be different for the different designs.

a. Concrete dips with and without shoulder protection cutoff walls and hand-placed loose riprap on the downstream side will require nominal maintenance. Mud, silt, gravel and stones will have to be removed from the surfaces of the dip sections during and after the rainy season by dozers and graders. Expansion, construction and contraction joints have been sealed with an approved asphalt joint sealer to prevent the penetration of water into the base and subbase materials. (Saturated base and subbase materials would permit the concrete slabs to settle or crack and in some instances require the removal of the damaged slabs, extensive subbase repair and the replacement of the concrete slab.) Base failures and cracked concrete slabs can be minimized if the 
joints are properly sealed. This will require the periodic inspection of all joints. The type of sealer used is not permanent and in a two- to four-year period will lose its elasticity and become hard or brittle, cracking will appear and the bond between the asphalt and the concrete will be broken. This will require cleaning (removing all of the old and brittle sealer) and resealing of the joints with an approved joint sealing compound. No drainage ditches or channels should be excavated within a 20-meter distance of the cutoff walls on the upstream side of the dip sections. Loose riprap on the downstream side will have to be repaired during and after the rainy season and, in some instances, additional widths of loose riprap to protect the cutoff walls will have to be installed to minimize downstream erosion.

b. DBST or asphalt-surfaced dips with cutoff walls only will require more maintenance and repair than the concrete dips. The flexible surface thickness is approximately $5 \mathrm{cM}$ and will not give the protection that rigid concrete provides. Mud, silt, gravel and stones will have to be removed from the surface during and after the rainy seasons. No track-mounted equipment, such as dozers, should be used to remove the debris but patrol graders can be used since this equipment is rubber-tired, and will not damage the asphalt paved surface. Caution must be used while removing the debris with the grader. The operator must be very careful and not permit the blade of the grader to come in contact with the asphalt. The grader blade should not be lowered below a height of $5 \mathrm{CM}$ above the surface of the pavement. The remaining silt and gravel should then be cleaned off by laborers using shovels and brooms. Upstream and downstream maintenance and repair will be similar to the concrete dips. The surface of the DBST dips will be subjected to damage caused by the gravel and large stones passing over the dips during the rainy season. Since the surface of the dip must be kept sealed to prevent erosion damage to the subbase and cutoff walls, the asphalt paving where damaged will have to be repaired with asphalt ( $\mathrm{RC}-2$ or $\mathrm{RC}-3)$ and crushed aggregate. This type of repair will, in all probability, become extensive and repetitious.

\section{c. The Maintenance Supervisor should consider not only} preventive maintenance but also an improvement program for the DBST dips. The cutoff walls on the upstream and downstream sides of the DBST dips have been installed at the required grades and elevations and are of the same size, location and nature as the cutoff walls for the concrete dips. Cutoff walls can be used as forms and grade controls for the placement of $17 \mathrm{CM}$ thick concrete slabs for the repair of heavily damaged sections of the asphalt surface. The Hari River offers an excellent source of sand and 
gravel aggregate and is readily accessible during the maintenance or dry season. The sand and gravel should be screened to a $2 \mathrm{CM}$ maximum size. Design mix for concrete should be approximately 3 parts aggregate, 2 parts sand and 1 part cement. No more than 20 liters of water per bag of cement should be allowed. By using the sand and gravel in its natural state, with oversize stones removed, a mix of 5 parts sand and gravel to 1 part cement will be suitable. This type of improvement, over a period of years, will eliminate much of the repetitious and time consuming maintenance.

\section{MAINTENANCE OF DBST OR ASPHALT PAVEMENT}

a. Flexible pavement of the type used for this project is an excellent and durable surface treatment provided that it is properly maintained. Flexible paving contributes no strength factor to the roadway but provides smooth, stabilized surface which protects the base and subbase materials. The base and subgrade materials must be kept dry or "pumping" by the base course will cause the surface of the roadway to become uneven and full of chuck holes. If allowed to remain unrepaired, these damaged areas will expand and soon the road will become impassable, then extensive and costly repairs will have to be made. No equipment for the reconstruction of large segments of the road is included in the recommended equipment 1 ist. As asphalt repaving will have to be done, a minimum amount of equipment for maintenance of the asphalt pavement should be procured. The following equipment is the minimum necessary:

(1) A 750-1300 1iter (200-500 gal) asphalt tank mounted on rubber tires (pul1 type) equipped with burners to heat the asphalt or keep it at a desirable temperature while in the field, circulating pump (gas driven) and hand spray.

(2) A 5- to 7-ton Tandem-steel wheel roller.

These two pieces of equipment will be adequate to keep the surface of the roadway in an acceptable state of repair.

b. The Maintenance Supervisor should be aware that he will have to be continually looking for signs of deterioration in the surface of the asphalt pavement. Timely repairs to the surface of the roadway will prevent extensive and costly repairs at a later date.

(1) One of the first signs of deterioration for this type of surface treatment will be lateral and longitudinal cracking 
appearing on the surface of the pavement. This is caused by oxidation, lack of traffic, overloaded trucks, lateral pressures at horizontal curves and base failures. This type of repair, if attended to at once, will be accomplished very easily and with very little cost. Only two pieces of equipment will be needed: the asphalt tank and a truck loaded with $1.5 \mathrm{~cm}$ minus crushed rock. Spray or distribute the asphalt (RC-2 or RC-3 heated to a temperature of approximately $55^{\circ} \mathrm{C}$ ) over the damaged section in the amounts of .7 to 1.1 liters per square lmeter. Spread the crushed rock by shovels over the asphalted area in a thin even mat and hand broom it to a smooth even transition with the adjacent surfaced areas. No roller will be needed for this operation. This type of repair will rejuvenate and reseal the surface of the pavement, protect the base and subbase materials and prevent further damage to the road. This skin patching or sealing is very necessary to preserve asphalt surfaced roadways.

(2) Base failures, chuck holes and broken pavement are the result of a lack of timely and sufficient maintenance. The sections of the roadway damaged to this extent will require excavation to a depth where the subbase or subgrade compaction and stability are the same as initially built. Adjacent edges of the undamaged sections of asphalt should be cut and trimmed vertically to a depth of $5 \mathrm{CM}$ to provide a key for the placement of the asphalt and aggregate. Subbase materials meeting the specifications (Appendix II) can be easily obtained in the nearby wadis and river beds. All stones over $4 \mathrm{CM}$ in size should be removed before material is placed in the affected areas. Backfill by placing the subbase material in layers of less than $15 \mathrm{CM}$ and compacting each layer by hand tampers or rubber-tired rollers to the density specified for the road (Appendix II) before placing another layer on it. This material is suitable to within $15 \mathrm{CM}$ of road surface. The top $15 \mathrm{CM}$ must meet the specifications for the base material. After the subbase has been compacted and stabilized, place the base material and compact it for an average depth of $15 \mathrm{~cm}$.

Crushed base aggregate stock piles left by the contractor may be found in considerable amounts at Stations 33, 59, 78, 94 and 108 . When the base aggregate has been backfilled and compacted, cover the surface of the base material with 1 liter per square meter of asphalt. Evenly distribute a layer of $2 \mathrm{CM}$ size crushed rock over the area, grade it to a smooth, even surface, then roll and compact it. Apply another 1 liter/sq meter of asphalt and then finish with a layer of $1.5 \mathrm{cM}$ crushed rock broomed and graded to a smooth, even surface and compacted with the roller. Small chuck holes and broken areas, where the damage does not exceed a depth of more than 10 to $15 \mathrm{CM}$, may be repaired by preparing a readymixed paving. Mix asphalt and aggregate with shovels or with a 
grader blade at a convenient location and haul it to the spots to be repaired in trucks. A design mix of $1.25 \mathrm{CM}$ minus aggregate with approximately 6 percent asphalt of the total weight of the aggregate should be used. The hole should first be cut vertically to a depth of $5 \mathrm{cM}$ with the square cut elongated with the flow of traffic. The area to be repaired should then be sealed with asphalt and the thoroughly pre-mixed asphalt and aggregate placed and compacted with hand tampers or the tires of a truck to a smooth, even transition with the adjacent surfaces.

c. With an adequate preventive maintenance program, the asphalt paved surface for this road is designed and constructed to provide a smooth, stable and durable traveled way for five (5) years. Plans should be made now to apply a new asphalt seal coat to the existing surface in about five (5) years. This seal coat will cost approximately $\$ 1,000$ (73,000 Afs) per kilometer if it is incorporated into and becomes a part of the preventive maintenance program.

\section{IMPROVEMENTS}

a. If washouts occur, showing that additional drainage would be desirable, more culvert pipes should be emplaced in the damaged section. This can most easily be done at the same time as the section is rebuilt. For this purpose a stock of culvert pipes should be built up; this can be easily done using unskilled labor at some central location. A suggested easily fabricated concrete form for this is shown in figures 5 and 6, attached. Aggregate can be collected from the numerous wadis or the stream bed of the Hari Rud. A well graded rock, cleaned of clay and other excess fines and screened to remove all rocks larger than $2 \mathrm{CM}$ should be used. A mix of 1 part cement, 5 parts aggregate (by weight) and not over 20 liters of water per bag of cement should be adequate. The thoroughly mixed concrete should be well tamped in the form with metal rods, to avoid holes, and be allowed to cure at least 24 hours before removing the form. Care must be taken to avoid breaking the curing pipes and they must be kept dampened for at least 7 days while curing. After a 28-day curing period, these pipes can be emplaced by merely placing them in the prepared trench, butted against each other, and backfilled. The backfill should be compacted to the density specified on figure 2 .

b. Loose riprap, large stones hand emplaced over an area liable to be eroded, can be placed around the inlets to culverts, below the outlets in the stream channel, on the surface of dikes and ditches, or wherever it is evident that erosion is occuring. This is a job that can be best accomplished by unskilled labor, 
can be done throughout the dry season, and will incur no cost but the labor cost. Care should be taken not to block existing drainage channels or structures, or to place large rocks in a manner that they could easily be dislodged and washed into the culverts. But, with these minimum precautions, the drainage structures of this road can be greatly improved by placing large amounts of riprap over all areas liable to be eroded.

c. Additional signs or other warning devices may be desirable. These too can be made from concrete and stockpiled for later use.

d. Other improvements will become obvious as the maintenance program is carried out and the maintenance engineer should be continually searching for ways to utilize his men and equipment to improve the road.

e. The present surface of the road is not made for heavy traffic or extremely long life. To preserve this highway as an effective link in the highway system it will be necessary to add to the surface from time to time. As a minimum it should be planned to add an additional surface treatment in five years, and at five-year intervals thereafter. If this is not done, the surface will oxidize to such an extent that the paving becomes filled with cracks and is no longer impervious to water. After a very short time the base will be saturated and further traffic will ruin the base and subbase so that a complete new road will be required. A surface treatment is much cheaper than a new road construction project.

\section{ICE AND SNOW REMOVAL}

During the winter months the surface of the road should be kept free of excessive loads of ice and snow. If allowed to stand for long periods of time on the surface, water will seep through saturating the base and subbase and thus causing areas of failure. The failures will appear in the spring, after the thaws and when traffic again becomes heavy, but the damage is done when the water accumulates.

a. Most areas of the road will be blown clean of snow and should not require cleaning. If a heavy snowstorm covers the entire area, motor graders or truck mounted plows may be used but extreme care must be taken by the operators not to damage the road surface. At least $2 \mathrm{CM}$ of clearance should be left between the blade on the equipment and the road. 
b. Drifts covering relatively short portions of the road can be easily removed by hand labor with shovels.

\section{ORGANIZATION}

a. A special organization headed by a civil engineer and staffed with trained supervisors, mechanics and operators is essential for adequate road maintenance. This organization should be directly under the Ministry of Public Works in Kabul and as independent of other local organizations as possible. An adequate staff of trained personnel can easily be recruited from the men who built this road if adequate salaries are provided. These men are now out of work but, in order to utilize their valuable talents, enough incentive must be given to them to come here off of their farms or from their shops in various bazaars. A workable organization is detailed in the attached figure.

b. Suggested salaries, based on the experience of the contractor who built this road, should be at least 1000 Afs per month for common laborers, 2300 Afs for operators, 2500 for mechanics and 3500 for supervisors.

c. Adequate fuel must be supplied for the equipment, as well as spare parts to keep it running. Any imagined savings in this area will be far more expensive in broken down equipment and ruined road; equipment that is sitting idle does no one any good.

d. Work cannot be done without supplies. Cement and asphalt must be made available when and as needed.

e. It should be anticipated that the maintenance of this road will cost no less than $\$ 40,000$ or $3,000,000$ Afs per year. This is based on the minimum figure of $\$ 300$ per $\mathrm{km}$ per year; in the U.S., the average cost per $\mathrm{km}$ of this type of road per year is closer to $\$ 900$. This money will be used to provide personnel salaries, supplies, spare parts and some replacement equipment. The detailed budget breakdown should be based on experience, and for this the maintenance engineer must keep detailed records of expenditures and requirements. About one-eighth of this budget will be for salaries, one-half for equipment operation (fuel, spare parts and tires), one-quarter for new equipment and the remaining three-eighths for supplies of cement and asphalt. 


\section{CONCLUSION}

a. It must be kept in mind that the maintenance program set out is for maintaining only the HIQ Road and does not include any excess for help to local villages, improvements to the city of Herat, or other possible worthwhile projects. If the effort is not to be diluted, and the road allowed to deteriorate as a result, this organization should be as completely free from the local government as it is possible to make it; the supervisor should report directly to PWD in Kabul and should not be given authority to utilize any of his men or equipment on anything but the HIQ Road. If local improvement projects are desirable, they must be provided for through other programs.

b. A good maintenance program will not only insure that the road remains in its current condition, it will also improve the roadway and drainage. Any road can be improved. As time and weather show the portions that are most critical, additional structures should be added. Many features of the road were designed to provide for adequate protection at a minimum cost. The information available to the road designers was scanty and experience will show where insufficient drainage structures or protection or channeling was provided. A good maintenance program will add these structures where they are required as well as repairing damaged areas. Road improvement should be continuous throughout the life of the road.

c. A $\$ 10,000,000$ road deserves some special care and attention. 


\section{APPENLIX XIIII}

\section{FINDING (FF FACT}

SUBJECT: Fxtensive Lamage to Intercentor Litches, Culverts and hoadway by Rainfall. Kecorded kainfall at Campite Station 73 was $0.69 "$.

1. a. Station $69+150$ to Station $71+800$.

Lamage to roaciway in this area can be attributed to the lact that culverts had not been installed at Station $69+150,70+486$ and $71+800$. Heavy runoff of water from existing wash at station $70+143$ overf'lowed road in area of Station 69+150. hunoff following the intercentor aitch cut through sige embankment at station $70+8 \mathrm{C}$ aown roadway, tr.rough cut to Station $71+100$ causing extensive erosion to subgrade between these two Stations.

b. Staticn 66+475: Existing wash which is a nronosea "J" section filled interceptor aitch ana overtlowed the road at this point. Corsiberable aamage to subgrade and subbase.

c. Station 63+650: Culvert at this Station had not been aaylighted either inlet or outlet, also aspearea underaesigned. kxtensive nonding noted. Also a ditch hao been cut through roaway at Station $64+000$ by Atghan locals, to prevent water from backing up into Tea House.

a. Station 62+807: Culvert at this Station had not been aaylightea either inlet or outlet causing water to overflow road and following aowngrade damaging roaway and sucbase. Haa dayliphting been completec culvert would have satisfactorily carriea monof.

e. Station 62+580: Existing wadi (not ä "j" (seticr.) filled and or cut through interceptor ditch overflowed roadway, causing consicerable damage to subfrade ano subbase in this area.

f. Station 62+417: Culvert at this Station had not been aaylighted, either inlet or outlet. Water overflowed road and followed downgrade to culvert at Station $62+807$ damaging roadway and subbase.

g. Station 57+683: Existing wadi filled and or cut through interceptor ditch overtlowing roadway aamaging subgrade and subbase. This is a proposed " $\mathrm{J}$ " section. 
h. Station 56+1900: Interceptor ditch could not carry runnoff, cut across rozdway about 200 meters west of culvert at Station $56+181$. Probable cause of failure for the interceptor ditch at this point was that the culvert could not sufficiently discharge runoff. Considerable damage in this area.

i. Station 55+131: Culvert at this Station had not been daylighted either inlet or outlet. Water overflowed roadway and followed downgrade toward culvert at Station 56+100. Also contractor did not follow interceptor ditch design at this Station allowing water to continue flowing in ditch to Culvert at Station 56+100. Considerable damage to subgrade and subbase. Had daylighting been in effect culvert would have been able to discharge munoff.

j. Station 54+175: Culvert at this Station had not been daylighted either inlet or outlet. Water overflowed roadway and followed downgrade toward Culvert at Station 55+131. Also contractor did not follow interceptor ditch design at this Station allowing runoff to continue in interceptor ditch to Culvert at Station 55+131, oroding ditch and overflowing road. Had daylighting been in effect culvert would have been able to discharge runoff.

k. Station 53+638: Existing wadi filled and or cut through interceptor ditch overflowing roadway - damaging subgrade and subbase. This is a proposed "J" section.

1. Station 51+175: Culvert at this Station was not of sufficient flow design to carry runoff. Water overflowed road from Station $50+900$ to Station 51+300, existing wadi at Station 50+592 which extends approximately 2 kilometers in a northeasterly direction intersecting a large wadi coming through gap in mountaln about 1 kilometer North of Station $59+600$, discharges a tremendous amount of water to this point. Extensive damage to subgrade and subbase occured in this area. Part of the runoff from this large wadi is also diverted to Box Culvert at Station $48+525$.

m. Station 4?+600: Sheet and channel flow cut through and filled up interceptor ditch at this point, overflowing and cutting through roaduay. Considerable damage to subgrade, subbase and base.

n. Station 47+000: Sheet and channel flow cut through and or filled up interceptor ditch, overflowing and cutting through roadway. Considerable damage to subgrade, subbase and base.

o. Station $27+800$ to Station 18+200: Complote failure of all culverts and interceptor ditches occured between these stations. Extensive damage to subgrade, subbase, base, culverts and interceptor ditches. 
p. Station $18+200$ to Station $17+640$ : Road completely washed out between these stations. Complete failure of cilverts and interceptor ditches.

q. Station $17+640$ to Station 0+000: Several minor areas were interceptor ditch filled up. Little if no flow or runoff between those stations - No damage in this area.

2. Summarization of investigation indicates that in the areas where the road was not damaged and the culverts and interceptor ditches functioned as designed lesser amount of rainfall and smaller areas of munoff were involved. Investigation also indicates that other areas where construction is now under way or proposed could, in all probability be subjected to the same conditions and similar damage as in the oforementioned aroas occur. "J" Jections (Hand placed grouted riprap) installed as indicated on the contrast plans functioned only where the runoff was slight. In areas of hesvy runoff and at right angles to the interceptor ditch the "J" sections filled up and allowed minoff from existing ditches to cut into or flow across roadway.

3. In conclusion, the extent of the damages incurred indicate that a redesign of drainage should be considered... 